Equações diferenciais ordinárias generalizadas e aplicações às equações diferenciais clássicas 


\title{
Equações diferenciais ordinárias generalizadas e aplicações às equações diferenciais clássicas
}

\author{
Eduard Toon
}

Orientadora: Profa. Dra. Márcia Cristina A. B. Federson

Tese apresentada ao Instituto de Ciências Matemáticas e de Computação - ICMC-USP, como parte dos requisitos para obtenção do título de Doutor em Ciências - Matemática . VERSÃO REVISADA 
Ficha catalográfica elaborada pela Biblioteca Prof. Achille Bassi e Seção Técnica de Informática, ICMC/USP, com os dados fornecidos pelo(a) autor(a)

\begin{tabular}{|c|c|}
\hline \multirow[t]{3}{*}{$\begin{array}{l}\text { TT668e } \\
e\end{array}$} & $\begin{array}{l}\text { Toon, Eduard } \\
\text { Equações diferenciais ordinárias generalizadas e } \\
\text { aplicações às equações diferenciais clássicas / Eduard } \\
\text { Toon; orientadora Márcia Cristina Anderson Braz } \\
\text { Federson. -- São Carlos, } 2012 \text {. } \\
\quad 89 \text { p. }\end{array}$ \\
\hline & $\begin{array}{l}\text { Tese (Doutorado - Programa de Pós-Graduação em } \\
\text { Matemática)-- Instituto de Ciências Matemáticas e } \\
\text { de Computação, Universidade de São Paulo, } 2012 \text {. }\end{array}$ \\
\hline & $\begin{array}{l}\text { 1. equações diferenciais ordinárias generalizadas. } \\
\text { 2. equações diferenciais funcionais em medida. } 3 . \\
\text { estabilidade. 4. comportamento assintótico de } \\
\text { soluções. 5. equações diferenciais ordinárias. I. } \\
\text { Federson, Márcia Cristina Anderson Braz, orient. II. } \\
\text { Título. }\end{array}$ \\
\hline
\end{tabular}



À minha familia, as pessoas mais importantes na minha vida. 


\section{Agradecimentos}

Agradeço, primeiramente, a minha família e familiares pelo apoio nas horas certas, palavras de incentivo, carinho, conselhos e compreensão com meus momentos de ausência.

Aos meus amigos e colegas pelo apoio, ajuda, discussões, risadas e companheirismo.

Agradeço à minha orientadora, professora Márcia Federson por seu trabalho de orientação, dedicação, paciência, pela contribuição em minha formação profissional, pelas palavras de incentivo e, também, pelas broncas, quando se fizeram necessárias. Agradeço, também, pela amizade que pudemos construir neste período.

Agradeço aos professores do ICMC-USP que contribuíram com minha formação.

Agradeço aos funcionários do ICMC-USP por proporcionarem um local adequado para o desenvolvimento deste trabalho.

E, por fim, agradeço a FAPESP pelo suporte financeiro durante a execução deste trabalho. 

O objetivo deste trabalho é estudar algumas propriedades de soluções de equações diferenciais ordinárias generalizadas e aplicar tais resultados a algumas equações diferenciais clássicas (equações diferenciais ordinárias abstratas e equações diferenciais funcionais em medida).

Os principais resultados tratam de existência-unicidade de soluções para uma classe de equações diferenciais ordinárias generalizadas, dependência contínua de soluções com respeito às condições iniciais e bacia de atração. Estes resultados são transferidos para uma classe de equações diferencias ordinárias abstratas. Também obtemos resultados sobre estabilidade da solução trivial de equações diferenciais ordinárias generalizadas e transferimos estes resultados para uma classe de equações diferenciais funcionais em medida. 

The purpose of this work is to study some properties of solutions of generalized ordinary differential equations and apply these results to some classical differential equations (abstract ordinary differential equations and measure functional differential equations).

The main results concern existence-uniqueness of a solution for a class of generalized ordinary differential equations, continuous dependence of solutions with respect to initial conditions and basin of attraction. These results are transfered to a class of abstract ordinary differential equations. We also obtain some results on the stability of the trivial solution of generalized ordinary differential equations and we transfer these results to a class of measure functional differential equations. 

Introdução $\quad$ xi

1 Equações Diferenciais Ordinárias Generalizadas Autônomas $\quad 1$

1.1 Teoria fundamental de EDOGs . . . . . . . . . . . . . . . . . . 1

1.2 EDOGs autônomas . . . . . . . . . . . . . . . . . 8

1.3 Bacia de atração para EDOGs autônomas . . . . . . . . . . . . . . . . 12

2 Aplicações às EDOs autônomas $\quad 19$

2.1 EDOGs autônomas x EDOs autônomas . . . . . . . . . . . . . . . . 19

2.2 Dependência contínua de soluções com respeito às condições iniciais 23

2.3 Bacia de atração para EDOs autônomas . . . . . . . . . . . . . . 25

3 Comportamento assintótico de EDFs em medida via EDOGs 29

3.1 Integração de Kurzweil-Henstock-Stieltjes . . . . . . . . . . . . . . . . . 30

3.2 Novos conceitos de estabilidade para EDFs em medida . . . . . . . . . 32

3.3 EDFs em medida vistas como EDOGs . . . . . . . . . . . . . . . . . 35

3.4 Relações entre conceitos de estabilidade para EDOGs e EDFs em medida . . . . . . . . . . . . . . . . . 46

4 Estabilidade de Lyapunov $\quad 57$

4.1 Estabilidade de Lyapunov para EDOGs . . . . . . . . . . . . . . . . . . 58

4.2 Estabilidade de Lyapunov para EDFs em medida via EDOGs . . . . . 63 
4.3 Outros resultados de estabilidade . . . . . . . . . . . . . . 71

$\begin{array}{ll}\text { Índice Remissivo } & 84\end{array}$

$\begin{array}{ll}\text { Referências Bibliográficas } & 87\end{array}$ 
A teoria de equações diferenciais ordinárias generalizadas (EDOGs) teve seu início em 1957, com o matemático tcheco Jaroslav Kurzweil. Sua ideia inicial era generalizar alguns resultados sobre dependência contínua, com relação a condições iniciais, de soluções de EDOs. Em seu trabalho pioneiro [22], Kurzweil definiu EDOGs para funções tomando valores em espaços euclidianos e de Banach.

A teoria de EDOGs está amplamente descrita em [25].

Uma das vantagens da teoria de EDOGs sobre a teoria clássica de equações diferenciais ordinárias pode ser vista no artigo [7] de Zvi Artstein. Ali foi mostrado que se considerarmos certas condições do tipo Carathéodory e Lipschitz sobre o lado direito das equações, ou seja, sobre as funções envolvidas, nem sempre obteremos resultados que pressupõem, por exemplo, a completude do "espaço das equações diferenciais", ou, equivalentemente, do espaço de funções que são lados direitos destas equações (identificando-se $\dot{x}=f(x, t)$ com $f$ ). De fato, considere a seguinte EDO

$$
\dot{x}=f(x, t), \quad\left(\dot{x}=\frac{d x}{d t}\right)
$$

e suponha que $f: \Omega \times \mathbb{R} \rightarrow \mathbb{R}^{n}$, com $\Omega \subset \mathbb{R}^{n}$ aberto, seja mensurável em $t$ e contínua em $x$. Considere a topologia caracterizada pela convergência

$$
f_{k} \rightarrow f_{0}, \quad \text { se } \quad \int_{0}^{t} f_{k}(x, s) d s \rightarrow \int_{0}^{t} f_{0}(x, s) d s, \quad(x, t) \in \Omega \times \mathbb{R}
$$

e as condições de Carathéodory e Lipschitz da seguinte forma 
(*) Para cada compacto $A \subset \Omega$, existe uma função Lebesgue integrável $M_{A}(t)$ tal que, para qualquer $x \in A$ vale

$$
|f(x, s)| \leq M_{A}(s)
$$

(**) Para cada compacto $A \subset \Omega$, existe uma função Lebesgue integrável $L_{A}(t)$ tal que, para quaisquer $x_{1}, x_{2} \in A$ vale

$$
\left|f\left(x_{1}, s\right)-f\left(x_{2}, s\right)\right| \leq L_{A}(s)\left|x_{1}-x_{2}\right|
$$

Artstein mostrou que o espaço de EDOs cujas funções do lado direito das equações satifazem as condições $(*)$ e $(* *)$ não contém todas as suas equações limites. De fato, seja $F_{0}$ uma função contínua que não é diferenciável em nenhum ponto e seja $F_{j}$, $j=1,2, \ldots$, uma sequência em $C^{1}$, que converge uniformemente para $F_{0}$. Se $f_{j}$ for a derivada de $F_{j}$, então para todo $(x, t), \int_{0}^{t} f_{j}(x, s) d s$ convergirá. Entretanto, o limite

$$
F_{0}(x, t)=\lim \int_{0}^{t} f_{j}(x, s) d s
$$

não possui uma representação integral $F_{0}(x, t)=\int_{0}^{t} f(x, s) d s$. Para cada $j=1,2, \ldots$, consideremos a equação

$$
\dot{x}=f_{j}(x, t)
$$

cuja solução é, a menos de uma constante, a primitiva $F_{j}$ de $f_{j}$. Note que, a "solução" limite $F_{0}$ não é solução de uma EDO.

O tipo de problema que acabamos de descrever não ocorre quando utilizamos a teoria de EDOGs. Veja [5] e [7].

Outra vantagem no estudo da teoria de EDOGs é que podemos obter a correspondência destas equações com outros tipos de equações diferenciais, tais como equações diferenciais ordinárias e equações diferenciais funcionais com retardamento (escrevemos EDFRs), onde reside nosso interesse e onde estão situados vários trabalhos.

A correspondência entre EDFRs e EDOGs foi investigada primeiramente em 1966 por Carlos Imaz e Z. Vorel e por F. Oliva e Z. Vorel sob certas hipóteses bastante técnicas (veja [21] e [24]). Em 2003, M. Federson e P. Z. Táboas mostraram, em [14], a mesma correspondência em um cenário mais próximo ao de [25]. Em tal trabalho, a teoria de EDOGs foi importante no estudo da dinâmica topológica de EDFRs. Em 2006, M. Federson e Š. Schwabik mostraram, em [12], que é possível relacionar EDFRs sob a ação de impulsos em tempos pré fixados e EDOGs. Recentemente, em 2011, S. M. 
Afonso estabeleceu, em [1], uma correspondência entre EDFRs sob a ação de impulsos em tempos variáveis e EDOGs. Veja também [2]. Todos estes trabalhos mostram que é possível obter resultados para EDFRs, com ou sem impulsos, que são mais gerais do que os resultados que já eram conhecidos na teoria clássica de EDFRs ou resultados inéditos, como os de [13] e [14], via teoria de EDOGs.

Como sabemos, o interesse no estudo de EDFRs está no fato de que estas equações descrevem muitos fenômenos físicos, biológicos e de engenharia, que envolvem um lapso de tempo entre causa e efeito. Já a teoria de equações diferenciais impulsivas descreve modelos em que ocorrem variações do estado em tempos tão pequenos que podem ser considerados instantâneos para efeitos de modelagem. Assim, uma teoria que possibilita a melhora de resultados sobre esses dois tipos de equação (que é o caso de EDOGs) é, de fato, muito interessante.

Este trabalho é dividido em duas partes: a primeira compreende os capítulos 1 e 2 e a segunda compreende os capítulos 3 e 4. A divisão foi feita desta maneira pois o Capítulo 2 trata de aplicações dos resultados do Capítulo 1. Já os capítulos 3 e 4 tratam, principalmente, de estabilidade de soluções de EDOGs e de EDFs em medida.

O Capítulo 1 é dividido em três seções. A primeira delas traz alguns resultados básicos sobre a teoria de EDOGs e a definição de uma nova classe de funções para EDOGs. Mostramos que podemos obter um teorema de existência e unicidade de solução para EDOGs, desde que a função envolvida na equação pertença a esta classe. Também mostramos que esta classe inclui uma classe para a qual já sabíamos haver um teorema de existência e unicidade de solução. Na segunda seção, definimos um conceito de EDOG autônoma que não existia na literatura. Enunciamos um teorema de existência e unicidade de solução para tais equações. Além disso, demonstramos alguns resultados sobre dependência contínua de soluções de EDOGs autônomas com relação às condições iniciais. Na última seção, descrevemos a noção de bacia de atração para EDOGs autônomas e mostramos que ela tem propriedades muito boas. Demonstramos resultados sobre estabilidade para pontos de equilíbrio via funcionais de Lyapunov e o resultado mais importante desta seção que garante a existência de um conjunto compacto contido na bacia de atração.

O Capítulo 2 está dividido em três seções. Na primeira seção, fazemos uma descrição de uma classe de problemas de EDOs autônomas tomando valores em espaços de Banach. Mostramos um resultado de existência e unicidade de solução para esta classe de problemas via EDOGs. Na segunda seção, obtemos resultados de dependência contínua de soluções de EDOs autônomas com relação às condições iniciais utilizando a relação obtida na primeira seção deste capítulo. Na última seção, apresentamos um 
conceito de bacia de atração para EDOs autônomas abstratas. Obtemos resultados similares aos que foram obtidos para a bacia de atração de EDOGs autônomas, utilizando a relação entre estas equações apresentada na primeira seção deste capítulo. Notamos que a importância do estudo de EDOs abstratas recai no fato de que algumas EDPs podem ser escritas como EDOs abstratas. Este não é o foco de nosso trabalho e o leitor interessado em tal fato pode consultar [6].

O terceiro capítulo é dividido em quatro seções. Na primeira seção, fazemos uma breve revisão sobre a integral de Kurzweil-Henstock-Stieltjes. Também apresentamos alguns resultados importantes sobre esta integral. Na segunda seção, descrevemos uma classe de problemas de equações diferenciais funcionais em medida. Além disso, apresentamos novos conceitos de estabilidade para tais equações. Na terceira seção, estabelemos uma relação biunívoca entre a classe de EDFs em medida apresentada na segunda seção deste capítulo e uma classe de EDOGs. Também demonstramos um resultado de existência e unicidade de solução para esta classe de EDFs em medida. Na última seção, definimos novos conceitos de estabilidade para EDOGs, a saber, estabilidade regular. Fazemos uma relação entre os novos conceitos de estabilidade para EDFs em medida, apresentados na segunda seção deste capítulo, e os novos conceitos de estabilidade para EDOGs. O estudo de EDFs em medida é interessante pois estas incluem equações impulsivas e também temos uma relação entre estas equações e equações em escalas temporais. O leitor interessado na relação entre EDFs em medida e equações em escalas temporais e também em mais fatos sobre equações em escalas temporais, pode consultar [9] e [10].

O Capítulo 4 é dividido em três partes. Na primeira seção, definimos o conceito de estabilidade de Lyapunov para EDOGs. Observamos que todos os conceitos de estabilidade para EDOGs conhecidos anteriormente ou mesmo os conceitos que definimos no Capítulo 3, levam em conta a variação ou o supremo das soluções. O conceito de estabilidade de Lyapunov apresentado aqui é diferente, pois não leva em conta a variação ou o supremo das soluções e, portanto, é um conceito mais simples de ser trabalhado e se aproxima mais das definições clássicas de estabilidade. Também demonstramos alguns resultados de estabilidade para EDOGs através de funcionais de Lyapunov. Na segunda seção, demonstramos resultados de estabilidade para EDFs em medida utilizando a relação entre estas equações e EDOGs e os resultados de estabilidade obtidos na primeira seção deste capítulo. Na última seção, apresentamos alguns resultados de estabilidade através de funcionais de Lyapunov para EDOGs e EDFs em medida. Estes resultados estão relacionados com os conceitos de estabilidade apresentados no Capítulo 3. 



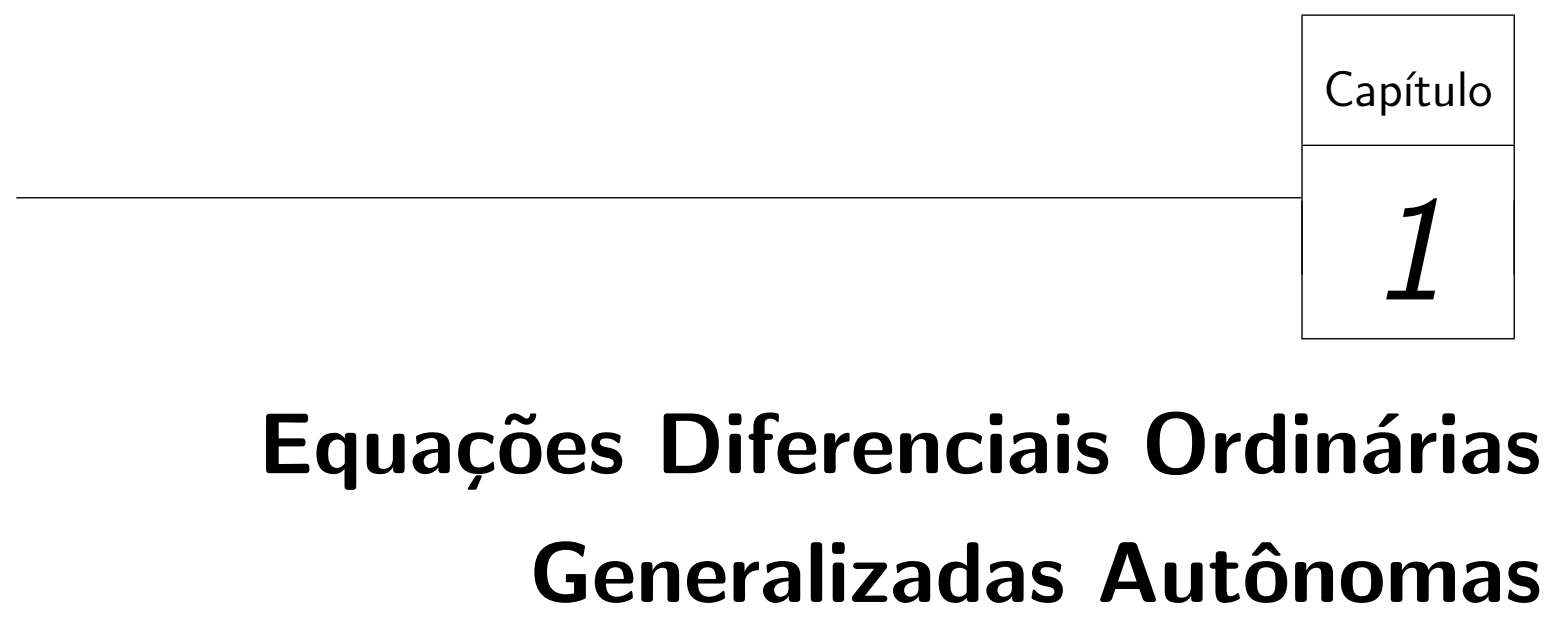

Neste capítulo, apresentamos alguns resultados básicos na teoria de equações diferenciais ordinárias generalizadas (EDOGs). Introduzimos uma nova classe de funções que podem ser lados direitos de EDOGs para as quais conseguiremos obter um resultado de existência-unicidade. Demonstramos, também, que esta classe inclui uma classe já conhecida na literatura para a qual tem-se um resultado de existência-unicidade.

Também introduzimos um conceito de EDOG autônoma que, até então, não era definido. Além disso, apresentamos resultados sobre dependência contínua de soluções com relação às condições iniciais e alguns resultados sobre o comportamento assintótico de soluções destas equações autônomas, como o conceito de bacia de atração para EDOGs autônomas.

\subsection{Teoria fundamental de EDOGs}

Nesta seção, relembramos conceitos básicos sobre EDOGs e apresentamos um teorema inédito sobre existência local e unicidade de solução para nossa nova classe de funções que são lado direito de EDOGs. 
Começamos esta seção lembrando o leitor da definição original da integral de Kurzweil apresentada em [22] e [23]. Veja, também, [25].

Uma divisão marcada de um intervalo compacto $[a, b] \subset \mathbb{R}$ é uma coleção finita de pares ponto-intervalo

$$
d=\left\{\left(\tau_{i},\left[s_{i-1}, s_{i}\right]\right): i=1,2, \ldots, k(d)\right\}
$$

onde $a=s_{0} \leq s_{1} \leq \ldots \leq s_{k(d)}=b$ é uma divisão de $[a, b]$ e $\tau_{i} \in\left[s_{i-1}, s_{i}\right]$, $i=$ $1,2, \ldots, k(d)$. Os pontos $\tau_{i}, i=1,2, \ldots, k(d)$, são chamados de marcas da divisão $d$.

Um calibre em $[a, b]$ é qualquer função $\delta:[a, b] \rightarrow(0,+\infty)$. Dado um calibre $\delta$ em $[a, b]$, uma divisão marcada $d=\left(\tau_{i},\left[s_{i-1}, s_{i}\right]\right)$ de $[a, b]$ será dita $\delta$-fina se, para quaisquer $i=1,2, \ldots, k(d)$, tivermos

$$
\left[s_{i-1}, s_{i}\right] \subset\left\{t \in[a, b]:\left|t-\tau_{i}\right|<\delta\left(\tau_{i}\right)\right\}
$$

No que segue, $X$ será um espaço de Banach com norma $\|\cdot\|$.

Uma função $U:[a, b] \times[a, b] \rightarrow X$ será dita Kurzweil integrável sobre o intervalo $[a, b]$, se existir um $I \in X$ tal que, dado $\varepsilon>0$, existe um calibre $\delta$ em $[a, b]$ tal que

$$
\left\|\sum_{i=1}^{k(d)}\left[U\left(\tau_{i}, \alpha_{i}\right)-U\left(\tau_{i}, \alpha_{i-1}\right)\right]-I\right\|<\varepsilon
$$

para toda divisão marcada $\delta$-fina $d=\left(\tau_{i},\left[\alpha_{i-1}, \alpha_{i}\right]\right)$ de $[a, b]$.

Vale a pena mencionar aqui que a integral de Kurzweil é linear, aditiva com respeito a intervalos adjacentes e vale a integrabilidade nos subintervalos de $[a, b]$. Quando $U(\tau, t)=f(\tau) t$, obtemos a integral de Perron usual e, mais geralmente, quando $U(\tau, t)=f(\tau) g(t)$, obtemos a integral de Perron-Stieltjes. Veja [27].

Com a definição da integral de Kurzweil em mente, apresentamos o conceito, definido pelo matemático tcheco Jaroslav Kurzweil em [22] e [23], de uma equação conhecida como equação diferencial ordinária generalizada, a qual nos referiremos simplesmente como EDOGs.

Algumas referências para a teoria de EDOGs são [2], [3], [10], [12], [13], [14], [22], [23] e, principalmente, [25].

Definição 1.1. Sejam $\mathcal{O} \subset X$ um aberto, $-\infty \leq a<b \leq \infty,[\alpha, \beta] \subset(a, b)$ um intervalo fechado e $\Omega=\mathcal{O} \times[\alpha, \beta]$. Uma função $x:[\alpha, \beta] \rightarrow X$ será dita uma solução 
da equação diferencial ordinária generalizada

$$
\frac{d x}{d \tau}=D G(x, t)
$$

no intervalo $[\alpha, \beta] \subset(a, b)$, se tivermos $(x(t), t) \in \Omega$ para $t \in[\alpha, \beta]$ e se valer a igualdade

$$
x(v)-x(\gamma)=\int_{\gamma}^{v} D G(x(\tau), s), \quad \gamma, v \in[\alpha, \beta]
$$

Observe que a notação em (1.1) é apenas simbólica. A letra $D$ indica que (1.1) é uma equação definida através das soluções da sua forma integral dada por (1.2).

Apresentamos, agora, uma classe de funções $G: \Omega \rightarrow X$ para as quais podemos obter algumas informações sobre as soluções de (1.1), em particular, existência e unicidade de soluções. Tal classe de funções também está descrita em [25].

Definição 1.2. Dada uma função não decrescente $h:[\alpha, \beta] \rightarrow \mathbb{R}$, diremos que uma função $G: \Omega \rightarrow X$ pertence à classe $\mathcal{F}(\Omega, h)$, se valerem as desigualdades

$$
\left\|G\left(x, s_{2}\right)-G\left(x, s_{1}\right)\right\| \leq\left|h\left(s_{2}\right)-h\left(s_{1}\right)\right|
$$

para quaisquer $\left(x, s_{2}\right),\left(x, s_{1}\right) \in \Omega e$

$$
\left\|G\left(x, s_{2}\right)-G\left(x, s_{1}\right)-G\left(y, s_{2}\right)+G\left(y, s_{1}\right)\right\| \leq\|x-y\|\left|h\left(s_{2}\right)-h\left(s_{1}\right)\right|
$$

para quaisquer $\left(x, s_{2}\right),\left(x, s_{1}\right),\left(y, s_{2}\right),\left(y, s_{1}\right) \in \Omega$.

Não é difícil demonstrar que, se $G: \Omega \rightarrow X$ satisfizer (1.3) e $x:[\alpha, \beta] \rightarrow X$ for uma solução de (1.1), então teremos

$$
\left\|x\left(s_{2}\right)-x\left(s_{1}\right)\right\| \leq\left|h\left(s_{2}\right)-h\left(s_{1}\right)\right|
$$

para quaisquer $s_{1}, s_{2} \in[\alpha, \beta]$. Para uma demonstração deste fato, veja o Lema $3.10 \mathrm{em}$ [25]. Em particular, qualquer ponto em $[\alpha, \beta]$ no qual a função $h$ é contínua também será um ponto de continuidade da solução $x:[\alpha, \beta] \rightarrow X$.

Se denotarmos por $\operatorname{var}_{\alpha}^{\beta}(x)$ a variação em $[\alpha, \beta]$ de uma função $x:[\alpha, \beta] \rightarrow X$ e assumirmos que $G: \Omega \rightarrow X$ satisfaz (1.3) e $x:[\alpha, \beta] \rightarrow X$ é uma solução de (1.1), então (1.5) implicará que $x$ é de variação limitada em $[\alpha, \beta]$ com

$$
\operatorname{var}_{\alpha}^{\beta} x \leq h(\beta)-h(\alpha)<+\infty
$$


Para mais detalhes, veja o Corolário 3.11 em [25].

Uma demonstração da proposição a seguir pode ser encontrada em [25], Lema 3.9.

Proposição 1.3. Suponha que $G: \Omega \rightarrow X$ satisfaça a condição (1.3). Se $[\alpha, \beta] \subset$ $(a, b)$ e $x:[\alpha, \beta] \rightarrow X$ for tal que $(x(t), t) \in \Omega$ para todo $t \in[\alpha, \beta]$ e se a integral $\int_{\alpha}^{\beta} D G(x(\tau), t)$ existir, então para quaisquer $s_{1}, s_{2} \in[\alpha, \beta]$, teremos

$$
\left\|\int_{s_{1}}^{s_{2}} D G(x(\tau), t)\right\| \leq\left|h\left(s_{2}\right)-h\left(s_{1}\right)\right| .
$$

O resultado a seguir nos dá condições sob as quais a integral envolvida na definição de solução da EDOG (1.1) existe. Para uma demonstração de tal resultado, veja [25], Teorema 3.14.

Teorema 1.4. Seja $G \in \mathcal{F}(\Omega, h)$. Se $x:[\alpha, \beta] \rightarrow X$, com $[\alpha, \beta] \subset(a, b)$, for o limite uniforme de uma sequência $\left(x_{k}\right)_{k \in \mathbb{N}}$ de funções escada $x_{k}:[\alpha, \beta] \rightarrow X$ tais que $(x(s), s)) \in \Omega$ e $\left(x_{k}(s), s\right) \in \Omega$, para todo $k \in \mathbb{N}$ e para todo $s \in[\alpha, \beta]$, então a integral $\int_{\alpha}^{\beta} D G(x(\tau), t)$ existirá e teremos

$$
\int_{\alpha}^{\beta} D G(x(\tau), t)=\lim _{k \rightarrow \infty} \int_{\alpha}^{\beta} D G\left(x_{k}(\tau), t\right) .
$$

Lembrando que $X$ denota um espaço de Banach com norma $\|\cdot\|$, seja $I \subset \mathbb{R}$ um intervalo qualquer da reta. Denotamos por $G(I, X)$ o espaço das funções regradas $f: I \rightarrow X$, ou seja, $G(I, X)$ é o espaço vetorial de todas as funções $f: I \rightarrow X$ tais que, para todo intervalo compacto $[a, b] \subset I$, os limites laterais

$$
f(t-)=\lim _{s \rightarrow t-} f(s) \quad \text { e } \quad f(t+)=\lim _{s \rightarrow t+} f(s)
$$

existem para todo $t \in[a, b]$, com

$$
f(a-)=f(a) \quad \text { e } \quad f(b+)=f(b) .
$$

Em $G(I, X)$, consideramos a topologia da convergência uniforme. Em particular, o espaço $G([a, b], X)$ é um espaço de Banach, quando dotado da norma usual do supremo, a qual denotamos por $\|\cdot\|_{\infty}$.

Sabemos que funções localmente de variação limitada são regradas. Também é verdade que qualquer função em $G([a, b], X)$ pode ser escrita como o limite uniforme de funções escada. Veja [19], Teorema I.3.1 para uma demonstração deste fato. 
Restringindo o Teorema 1.4 a funções regradas $x:[\alpha, \beta] \rightarrow X$, obtemos a proposição seguinte.

Proposição 1.5. Sejam $G \in \mathcal{F}(\Omega, h), x:[\alpha, \beta] \rightarrow X$ regrada (em particular, uma função de variação limitada) $e(x(s), s) \in \Omega$ para todo $s \in[\alpha, \beta]$. Então a integral $\int_{\alpha}^{\beta} D G(x(\tau), t)$ existe e a função $s \mapsto \int_{\alpha}^{s} D G(x(\tau), t) \in X$ é de variação limitada em $[\alpha, \beta]$ (e, portanto, também regrada).

O próximo teorema é um resultado fundamental na teoria de EDOGs. Ele garante a existência local e unicidade de uma solução da equação (1.1), sempre que $G: \Omega \rightarrow$ $X$ pertencer à classe $\mathcal{F}(\Omega, h)$, com $h$ não decrescente e contínua à esquerda. Uma demonstração deste resultado pode ser encontrada em [12], Teorema 2.15.

Teorema 1.6. Suponha que $G: \Omega \rightarrow X$ pertença à classe $\mathcal{F}(\Omega, h)$, onde a função $h$ é não decrescente e contínua à esquerda. Se dado $\left(\widetilde{x}, t_{0}\right) \in \Omega$ tal que para $\widetilde{x}_{+}=$ $\widetilde{x}+G\left(\widetilde{x}, t_{0}+\right)-G\left(\widetilde{x}, t_{0}\right)$, tivermos $\left(\widetilde{x}_{+}, t_{0}\right) \in \Omega$, então existirão $\Delta>0$ e uma única solução $x:\left[t_{0}, t_{0}+\Delta\right] \rightarrow X$ da equação diferencial ordinária generalizada (1.1) para a qual $x\left(t_{0}\right)=\widetilde{x}$.

Fazendo uma pequena modificação na Definição 1.2, obtemos uma classe maior de funções $G: \Omega \rightarrow X$ para a qual ainda conseguimos garantir existência local e unicidade de uma solução da EDOG (1.1). Introduzimos, então, esta classe a seguir.

Vamos usar a notação $x \in G([\alpha, \beta], \mathcal{O})$ para uma função $x \in G([\alpha, \beta], X)$ tal que $x(s) \in \mathcal{O}$, para todo $s \in[\alpha, \beta]$, onde $\mathcal{O}$ é um aberto de $X$.

Definição 1.7. Dada uma função não decrescente $h:[\alpha, \beta] \rightarrow \mathbb{R}$, diremos que uma função $G: \Omega \rightarrow X$ pertence à classe $\widetilde{\mathcal{F}}(\Omega, h)$, se a integral $\int_{\alpha}^{\beta} D G(x(\tau)$, s) existir para qualquer $x \in G([\alpha, \beta], \mathcal{O})$, e se tivermos

$$
\left\|\int_{s_{1}}^{s_{2}} D G(z(\tau), s)\right\| \leq\left|h\left(s_{2}\right)-h\left(s_{1}\right)\right|
$$

$e$

$$
\left\|\int_{s_{1}}^{s_{2}} D[G(z(\tau), s)-G(w(\tau), s)]\right\| \leq\|z-w\|_{\infty}\left|h\left(s_{2}\right)-h\left(s_{1}\right)\right|
$$

para quaisquer $z, w \in G([\alpha, \beta], \mathcal{O})$ e $s_{1}, s_{2} \in[\alpha, \beta]$.

Note que, pela Proposição 1.5, se (1.3) for verificada, então a existência das integrais em (1.7) e (1.8) estará garantida.

O resultado a seguir garante que $\mathcal{F}(\Omega, h) \subset \widetilde{\mathcal{F}}(\Omega, h)$. 
Proposição 1.8. Suponha que $G \in \mathcal{F}(\Omega, h)$. Então para quaisquer $z, w \in G([\alpha, \beta], \mathcal{O})$ e quaisquer $s_{1}, s_{2} \in[\alpha, \beta]$, valem (1.7) e (1.8), ou seja, $G \in \widetilde{F}(\Omega, h)$.

Demonstração: Suponha que $G \in \mathcal{F}(\Omega, h)$.

Sejam $z, w \in G([\alpha, \beta], \mathcal{O})$ funções dadas e $s_{1}, s_{2} \in[\alpha, \beta]$ com $s_{1}<s_{2}$. Pela Proposição 1.5, as integrais $\int_{\alpha}^{\beta} D G(z(\tau), s)$ e $\int_{\alpha}^{\beta} D G(w(\tau), s)$ existem e, pela Proposição 1.3 obtemos (1.7). Assim, resta mostrar que (1.8) também vale.

Pela propriedade de integrabilidade em subintervalos, dado $\epsilon>0$, existe um calibre $\delta$ em $\left[s_{1}, s_{2}\right]$ tal que, para toda divisão marcada $\delta$-fina $d=\left(\tau_{i},\left[t_{i-1}, t_{i}\right]\right)$ de $\left[s_{1}, s_{2}\right]$, valem as desigualdades

$$
\left\|\int_{s_{1}}^{s_{2}} D G(z(\tau), t)-\sum_{i=1}^{k(d)}\left[G\left(z\left(\tau_{i}\right), t_{i}\right)-G\left(z\left(\tau_{i}\right), t_{i-1}\right)\right]\right\|<\epsilon
$$

e

$$
\left\|\int_{s_{1}}^{s_{2}} D G(w(\tau), t)-\sum_{i=1}^{k(d)}\left[G\left(w\left(\tau_{i}\right), t_{i}\right)-G\left(w\left(\tau_{i}\right), t_{i-1}\right)\right]\right\|<\epsilon .
$$

Assim,

$$
\begin{aligned}
& \left\|\int_{s_{1}}^{s_{2}}[D G(z(\tau), t)-D G(w(\tau), t)]\right\| \\
\leq & \left\|\int_{s_{1}}^{s_{2}} D G(z(\tau), t)-\sum_{i=1}^{k(d)}\left[G\left(z\left(\tau_{i}\right), t_{i}\right)-G\left(z\left(\tau_{i}\right), t_{i-1}\right)\right]\right\| \\
+ & \left\|\int_{s_{1}}^{s_{2}} D G(w(\tau), t)-\sum_{i=1}^{k(d)}\left[G\left(w\left(\tau_{i}\right), t_{i}\right)-G\left(w\left(\tau_{i}\right), t_{i-1}\right)\right]\right\| \\
+ & \sum_{i=1}^{k(d)}\left\|G\left(z\left(\tau_{i}\right), t_{i}\right)-G\left(z\left(\tau_{i}\right), t_{i-1}\right)-G\left(w\left(\tau_{i}\right), t_{i}\right)+G\left(w\left(\tau_{i}\right), t_{i-1}\right)\right\| \\
< & 2 \epsilon+\sum_{i=1}^{k(d)}\left\|z\left(\tau_{i}\right)-w\left(\tau_{i}\right)\right\|\left[h\left(t_{i}\right)-h\left(t_{i-1}\right)\right] \leq 2 \epsilon+\|z-w\|_{\infty}\left[h\left(s_{2}\right)-h\left(s_{1}\right)\right] .
\end{aligned}
$$

Daí, pela arbitrariedade de $\epsilon>0$, obtemos

$$
\left\|\int_{s_{1}}^{s_{2}}[D G(z(\tau), t)-D G(w(\tau), t)]\right\| \leq\|z-w\|_{\infty}\left[h\left(s_{2}\right)-h\left(s_{1}\right)\right]
$$

o que completa a demonstração. 
O resultado a seguir garante a existência local e unicidade de uma solução da equação (1.1) para $G$ na classe $\widetilde{\mathcal{F}}(\Omega, h)$, com $h$ não decrescente e contínua à esquerda.

Teorema 1.9. Suponha que $G: \Omega \rightarrow X$ satisfaça (1.3) e pertença à classe $\widetilde{\mathcal{F}}(\Omega, h)$, onde a função $h$ é não decrescente e contínua à esquerda. Se dado $\left(\widetilde{x}, t_{0}\right) \in \Omega$ tal que para $\widetilde{x}_{+}=\widetilde{x}+G\left(\widetilde{x}, t_{0}+\right)-G\left(\widetilde{x}, t_{0}\right)$, tivermos $\left(\widetilde{x}_{+}, t_{0}\right) \in \Omega$, então existirão $\Delta>0$ e uma única solução $x:\left[t_{0}, t_{0}+\Delta\right] \rightarrow X$ da equação diferencial ordinária generalizada (1.1) tal que $x\left(t_{0}\right)=\widetilde{x}$.

Demonstração: Esta demonstração faz uso das principais ideias da demonstração do Teorema 2.15 de [12].

Primeiramente, consideramos $t_{0}$ como um ponto de continuidade da função $h$, ou seja, $h\left(t_{0}+\right)=h\left(t_{0}\right)$. Suponha que $\Delta>0$ seja tal que $\left[t_{0}, t_{0}+\Delta\right] \subset[\alpha, \beta]$ e $h\left(t_{0}+\Delta\right)-$ $h\left(t_{0}\right)<\frac{1}{2}$ e também que $\|x-\widetilde{x}\| \leq h\left(t_{0}+\Delta\right)-h\left(t_{0}\right)$ implique $x \in \mathcal{O}$.

Seja $Q$ o conjunto das funções $z:\left[t_{0}, t_{0}+\Delta\right] \rightarrow X$ tais que $z \in G\left(\left[t_{0}, t_{0}+\Delta\right], X\right)$ e $\|z(t)-\widetilde{x}\| \leq h(t)-h\left(t_{0}\right)$ para $t \in\left[t_{0}, t_{0}+\Delta\right]$. Então o conjunto $Q \subset G\left(\left[t_{0}, t_{0}+\Delta\right], X\right)$ é fechado.

Para cada $z \in Q$, definimos

$$
T z(s)=\widetilde{x}+\int_{t_{0}}^{s} D G(z(\tau), t), \quad s \in\left[t_{0}, t_{0}+\Delta\right] .
$$

Como $G$ pertence à classe $\widetilde{F}(\Omega, h)$ e, portanto, a integral $\int_{t_{0}}^{t_{0}+\Delta} D G(x(\tau), t)$ existe para todo $x \in G\left(\left[t_{0}, t_{0}+\Delta\right], X\right)$ o operador $T$ está bem definido. Note que, o fato de $G \in \widetilde{\mathcal{F}}(\Omega, h)$, implica que

$$
\|T z(s)-\widetilde{x}\|=\left\|\int_{t_{0}}^{s} D G(z(\tau), t)\right\| \leq h(s)-h\left(t_{0}\right), \quad s \in\left[t_{0}, t_{0}+\Delta\right]
$$

Logo $T$ aplica elementos de $Q$ em $Q$.

Sejam $z, w \in Q$ dados. Então, usando (1.8), para todo $s \in\left[t_{0}, t_{0}+\Delta\right]$, temos

$$
\| T z(s)-T w(s)]\|=\| \int_{t_{0}}^{s} D[G(z(\tau), t)-G(w(\tau), t)]\|\leq\| z-w \|_{\infty}\left[h(s)-h\left(t_{0}\right)\right] .
$$

Portanto

$$
\|T z-T w\|_{\infty} \leq\|z-w\|_{\infty}\left[h\left(t_{0}+\Delta\right)-h\left(t_{0}\right)\right]<\frac{1}{2}\|z-w\|_{\infty}
$$

e, assim, $T$ é uma contração. O resultado segue, então, pelo Teorema do Ponto Fixo de Banach. 
Consideremos, agora, o caso em que $t_{0}$ não é um ponto de continuidade de $h$. Defina $\widetilde{h}(t)=h(t)$ para $t \leq t_{0}$ e $\widetilde{h}(t)=h(t)-h\left(t_{0}+\right)$ para $t>t_{0}$. Então a função $\widetilde{h}$ é contínua em $t_{0}$ e não decrescente. Definindo $\widetilde{G}(x, t)=G(x, t)$ para $t \leq t_{0}$ e $\widetilde{G}(x, t)=G(x, t)-\left[G\left(\widetilde{x}, t_{0}+\right)-G\left(\widetilde{x}, t_{0}\right)\right]$ para $t>t_{0}$, é fácil ver que $\widetilde{G} \in \widetilde{\mathcal{F}}(\Omega, \widetilde{h})$

e, como no caso anterior, existe uma solução $z$ de $\frac{d z}{d \tau}=D \widetilde{G}(z, t) \operatorname{com} z\left(t_{0}\right)=\widetilde{x}_{+}$. Finalmente, definindo $x\left(t_{0}\right)=\widetilde{x}$ e $x(t)=z(t)$ para $t>t_{0}$, temos uma solução de (1.1) tal que $x\left(t_{0}\right)=\widetilde{x}$ e a demonstração está completa.

\subsection{EDOGs autônomas}

Introduziremos, nesta seção, o conceito de EDOGs autônomas e apresentaremos alguns resultados básicos.

Definição 1.10. Uma equação diferencial ordinária generalizada autônoma é uma equação da forma

$$
\frac{d x}{d \tau}=D[G(x, t)]
$$

onde $G: \Omega \rightarrow X, \Omega=\mathcal{O} \times[\alpha, \beta]$, é dada por

$$
G(x, t)=F(x) t
$$

$\operatorname{com} F: \mathcal{O} \rightarrow X$ et $\in[\alpha, \beta]$, onde $\mathcal{O} \subset X$ é um aberto.

Neste momento, o leitor pode achar estranho o nome "autônoma" para uma equação do tipo

$$
\frac{d x}{d \tau}=D[F(x) t]
$$

com este $t$ "aparecendo" no lado direito da equação. Entretanto, lembramos o leitor que uma solução de (1.10) é qualquer função $x:[\alpha, \beta] \rightarrow X$ tal que $x(t) \in \mathcal{O}$ para todo $t \in[\alpha, \beta]$ e vale

$$
x(v)-x(\gamma)=\int_{\gamma}^{v} D[F(x(\tau)) t]=\int_{\gamma}^{v} F(x(t)) d t
$$

para quaisquer $\gamma, v \in[\alpha, \beta]$, onde a última integral é exatamente a integral de Perron de $F: \mathcal{O} \rightarrow X$. Assim, a forma integral

$$
x(t)=x(\alpha)+\int_{\alpha}^{t} F(x(s)) d s, \quad t \in[\alpha, \beta],
$$


correspondente a (1.10) torna claro porque o nome "autônoma" de fato faz sentido.

É importante observar que o processo definido pela solução da equação (1.10) será um processo autônomo, ou semigrupo e, portanto, o nome autônoma faz sentido para a equação (1.10). Este fato será mostrado na Seção 1.3.

Note que, se $G \in \widetilde{\mathcal{F}}(\Omega, h)$, com $G(x, t)=F(x) t$, então valem as seguintes condições

(i) Para qualquer $x \in G([\alpha, \beta], \mathcal{O})$, a integral de Perron $\int_{\alpha}^{\beta} F(x(s)) d s$ existe;

(ii) Para quaisquer $z \in G([\alpha, \beta], \mathcal{O})$ e $s_{1}, s_{2} \in[\alpha, \beta]$,

$$
\left\|\int_{s_{1}}^{s_{2}} F(z(s)) d s\right\|<\left|h\left(s_{2}\right)-h\left(s_{1}\right)\right|
$$

(iii) Para quaisquer $z, w \in G([\alpha, \beta], \mathcal{O})$ e $s_{1}, s_{2} \in[\alpha, \beta]$,

$$
\left\|\int_{s_{1}}^{s_{2}}[F(z(s))-F(w(s))] d s\right\|<\|z-w\|\left|h\left(s_{2}\right)-h\left(s_{1}\right)\right| .
$$

O teorema que segue é um caso particular do Teorema 1.9 sobre existência-unicidade, agora particularizando para EDOGs autônomas.

Teorema 1.11. Nas condições (i), (ii) e (iii) acima, dado $\left(\widetilde{x}, t_{0}\right) \in \Omega$, existem $\Delta>0$ e uma única solução $x:\left[t_{0}, t_{0}+\Delta\right] \rightarrow X$ da EDOG autônoma (1.10) tal que $x\left(t_{0}\right)=\widetilde{x}$.

Ainda sob as condições (i), (ii) e (iii), podemos demonstrar alguns resultados sobre dependência contínua de soluções de EDOGs autônomas, com respeito a parâmetros. Os três resultados a seguir são adaptações de resultados de [5] para o contexto de EDOGs autônomas.

Lembramos o leitor que $\mathcal{O}$ denota um aberto do espaço de Banach $X$.

Diremos que uma sequência de funções $F_{k}: \mathcal{O} \rightarrow X$ converge para uma função $F_{0}: \mathcal{O} \rightarrow X$ em $\widetilde{\mathcal{F}}(\Omega, h)$, quando tivermos

$$
\left\|\int_{\alpha}^{\beta}\left[F_{k}(x(s))-F_{0}(x(s))\right] d s\right\| \stackrel{k \rightarrow+\infty}{\longrightarrow} 0
$$

para cada $x \in G([\alpha, \beta], \mathcal{O})$.

Lema 1.12. Suponha que, para cada $k=0,1, \ldots$, a função $F_{k}: \mathcal{O} \rightarrow X$ satisfaça as condições (i), (ii) e (iii). Suponha, também, que $F_{k} \stackrel{k \rightarrow \infty}{\longrightarrow} F_{0}$, e que, para cada 
$k=1,2, \ldots, \psi_{k} \in G([\alpha, \beta], \mathcal{O})$. Suponha, ainda, que $\psi_{0}:[\alpha, \beta] \rightarrow X$ seja tal que $\psi_{0}(s) \in \mathcal{O}$, para todo $s \in[\alpha, \beta]$, com

$$
\left\|\psi_{k}-\psi_{0}\right\|_{\infty} \stackrel{k \rightarrow+\infty}{\longrightarrow} 0
$$

Então

$$
\left\|\int_{\alpha}^{\beta}\left[F_{k}\left(\psi_{k}(s)\right)-F_{0}\left(\psi_{0}(s)\right)\right] d s\right\| \stackrel{k \rightarrow+\infty}{\longrightarrow} 0 .
$$

Demonstração: Seguiremos, aqui, a ideia da demonstração do Lema A.1 de [5].

Note que $\psi_{0} \in G([\alpha, \beta], X)$, pois $\psi_{0}$ é, por hipótese, o limite uniforme de funções regradas em $[\alpha, \beta]$. Então, pela condição (ii), as integrais $\int_{\alpha}^{\beta} F_{k}\left(\psi_{k}(s)\right) d s, k=0,1,2, \ldots$, existem.

Seja $\varepsilon>0$ dado. Então a função regrada $\psi_{0} \in G([\alpha, \beta], X)$ pode ser uniformemente aproximada por uma função escada (veja [19], Teorema I.3.1), isto é, existe uma função escada $y:[\alpha, \beta] \rightarrow X$, tal que

$$
\left\|y-\psi_{0}\right\|_{\infty}=\sup _{\alpha \leq t \leq \beta}\left\|y(t)-\psi_{0}(t)\right\|<\varepsilon
$$

Como $\left\|\psi_{k}-\psi_{0}\right\|_{\infty} \stackrel{k \rightarrow+\infty}{\longrightarrow} 0$, existe um inteiro positivo $N_{0}$ tal que

$$
\left\|\psi_{k}-\psi_{0}\right\|_{\infty}<\varepsilon
$$

para todo $k>N_{0}$.

Também vale

$$
\begin{gathered}
\left\|\int_{\alpha}^{\beta}\left[F_{k}\left(\psi_{k}(s)\right)-F_{0}\left(\psi_{0}(s)\right)\right] d s\right\| \leq\left\|\int_{\alpha}^{\beta}\left[F_{k}\left(\psi_{k}(s)\right)-F_{k}\left(\psi_{0}(s)\right)\right] d s\right\| \\
+\left\|\int_{\alpha}^{\beta}\left[F_{k}\left(\psi_{0}(s)\right)-F_{k}(y(s))\right] d s\right\|+\left\|\int_{\alpha}^{\beta}\left[F_{k}(y(s))-F_{0}(y(s))\right] d s\right\|+ \\
+\left\|\int_{\alpha}^{\beta}\left[F_{0}(y(s))-F_{0}\left(\psi_{0}(s)\right)\right] d s\right\| .
\end{gathered}
$$

Considerando $k>N_{0}$ e usando a condição (iii) na primeira integral ao lado direito da desigualdade (1.14), obtemos

$$
\left\|\int_{\alpha}^{\beta}\left[F_{k}\left(\psi_{k}(s)\right)-F_{k}\left(\psi_{0}(s)\right)\right] d s\right\| \leq\left\|\psi_{k}-\psi_{0}\right\|_{\infty}[h(\beta)-h(\alpha)] \leq \varepsilon[h(\beta)-h(\alpha)] .
$$


Analogamente, tomando $k>N_{0}$ e considerando o segundo e quarto termos ao lado direito de (1.14), temos as desigualdades

$$
\left\|\int_{\alpha}^{\beta}\left[F_{k}\left(\psi_{0}(s)\right)-F_{k}(y(s))\right] d s\right\|<\varepsilon[h(\beta)-h(\alpha)]
$$

e

$$
\left\|\int_{\alpha}^{\beta}\left[F_{0}(y(s))-F_{0}\left(\psi_{0}(s)\right)\right] d s\right\|<\varepsilon[h(\beta)-h(\alpha)] .
$$

Assim, para $k>N_{0}$, obtemos

$$
\begin{gathered}
\left\|\int_{\alpha}^{\beta}\left[F_{k}\left(\psi_{k}(s)\right)-F_{0}\left(\psi_{0}(s)\right)\right] d s\right\|< \\
<3 \varepsilon[h(\beta)-h(\alpha)]+\left\|\int_{\alpha}^{\beta}\left[F_{k}(y(s))-F_{0}(y(s))\right] d s\right\| .
\end{gathered}
$$

Consideremos, então, a integral $\int_{\alpha}^{\beta}\left[F_{k}(y(s))-F_{0}(y(s))\right] d s$.

Como $y:[\alpha, \beta] \rightarrow X$ é uma função escada, então $y$ é regrada. Por hipótese, $F_{k} \stackrel{k \rightarrow \infty}{\longrightarrow} F_{0}$. Logo,

$$
\left\|\int_{\alpha}^{\beta}\left[F_{k}(y(s))-F_{0}(y(s))\right] d s\right\| \stackrel{k \rightarrow \infty}{\longrightarrow} 0
$$

o que conclui a demonstração.

A seguir usaremos $B V([\alpha, \beta], \mathcal{O})$ para denotar o subconjunto de $B V([\alpha, \beta], X)$ das funções de variação limitada de $[\alpha, \beta]$ em $X$ que assumem valores em $\mathcal{O} \subset X$, onde $\mathcal{O}$ é um aberto de $X$.

Corolário 1.13. Suponha que, para cada $k=0,1, \ldots$, a função $F_{k}: \mathcal{O} \rightarrow X$ satisfaça as condições (i), (ii) e (iii) e $F_{k} \stackrel{k \rightarrow+\infty}{\longrightarrow} F_{0}$. Para cada $k=1,2, \ldots$, sejam $\psi_{k} \in$ $B V([\alpha, \beta], \mathcal{O})$. Suponha, também, que $\psi_{0}:[\alpha, \beta] \rightarrow X$ seja tal que $\psi_{0}(s) \in \mathcal{O}$ para todo $s \in[\alpha, \beta]$ e que $\left\|\psi_{k}-\psi_{0}\right\|_{B V} \stackrel{k \rightarrow+\infty}{\longrightarrow} 0$. Então

$$
\left\|\int_{\alpha}^{\beta}\left[F_{k}\left(\psi_{k}(s)\right)-F_{0}\left(\psi_{0}(s)\right)\right] d s\right\| \stackrel{k \rightarrow+\infty}{\longrightarrow} 0 .
$$

Demonstração: Esta demonstração segue as ideias da demonstração do Corolário A.2 de [5].

Sabe-se que $B V([\alpha, \beta], \mathcal{O}) \subset G([\alpha, \beta], \mathcal{O})$ e que $B V([\alpha, \beta], X)$ munido da norma da variação é completo (veja [19]). 
Além disso, para todo $t \in[\alpha, \beta]$, temos

$$
\begin{aligned}
\left\|\psi_{k}(t)-\psi_{0}(t)\right\| & \leq\left\|\psi_{k}(\alpha)-\psi_{0}(\alpha)\right\|+\left\|\psi_{k}(t)-\psi_{0}(t)-\left(\psi_{k}(\alpha)-\psi_{0}(\alpha)\right)\right\| \\
& \leq\left\|\psi_{k}(\alpha)-\psi_{0}(\alpha)\right\|+\operatorname{var}_{\alpha}^{t}\left(\psi_{k}-\psi_{0}\right) \\
& \leq\left\|\psi_{k}(\alpha)-\psi_{0}(\alpha)\right\|+\operatorname{var}_{\alpha}^{\beta}\left(\psi_{k}-\psi_{0}\right)=\left\|\psi_{k}-\psi_{0}\right\|_{B V},
\end{aligned}
$$

onde $\operatorname{var}_{\alpha}^{t} f$ denota a variação de uma função $f$ no intervalo $[\alpha, t]$. Assim $\| \psi_{k}-$ $\psi_{0} \|_{\infty} \stackrel{k \rightarrow+\infty}{\longrightarrow} 0$ e o resultado segue pelo Lema 1.12.

Por fim, enunciamos um resultado sobre dependência contínua de soluções de EDOGs autônomas com relação às condições iniciais. Sua demonstração será omitida, pois é similar à demonstração da Proposição A.3 de [5].

Proposição 1.14. Suponha que, para cada $k=0,1, \ldots$, a função $F_{k}: \mathcal{O} \rightarrow X$ satisfaça as condições (i), (ii), (iii) e $F_{k} \stackrel{k \rightarrow \infty}{\longrightarrow} F_{0}$. Para cada $k=1,2, \ldots$, seja $x_{k}:[\alpha, \beta] \rightarrow \mathcal{O}$ uma solução da EDOG autônoma

$$
\frac{d x}{d \tau}=D\left[F_{k}(x) t\right]
$$

em $[\alpha, \beta]$ e suponha que

$$
\lim _{k \rightarrow+\infty} x_{k}(s)=x_{0}(s), \quad s \in[\alpha, \beta],
$$

e $x_{0}(s) \in \mathcal{O}$, para todo $s \in[\alpha, \beta]$. Então $x_{0}:[\alpha, \beta] \rightarrow \mathcal{O}$ satisfaz:

- $\left\|x_{0}\left(s_{2}\right)-x_{0}\left(s_{1}\right)\right\| \leq\left|h\left(s_{2}\right)-h\left(s_{1}\right)\right|$, para quaisquer $s_{1}, s_{2} \in[\alpha, \beta]$;

- $\lim _{k \rightarrow+\infty} x_{k}(s)=x_{0}(s)$ uniformemente em $[\alpha, \beta]$;

- $x_{0}$ é uma solução da EDOG autônoma $\frac{d x}{d \tau}=D\left[F_{0}(x) t\right]$ em $[\alpha, \beta]$.

\subsection{Bacia de atração para EDOGs autônomas}

Nesta seção, apresentaremos um conceito de bacia de atração para EDOGs autônomas. Demonstraremos alguns resultados interessantes para estas equações, tais como o fato da bacia de atração ser não vazia e aberta e também mostraremos um resultado sobre estabilidade de pontos de equilíbrio através de funcionais de Lyapunov.

Sejam $X$ um espaço de Banach, $\mathcal{O} \subset X$ um conjunto aberto arbitrário e $F: \mathcal{O} \rightarrow X$ uma aplicação qualquer. 


\section{Considere a EDOG autônoma}

$$
\frac{d x}{d \tau}=D[F(x) t], \quad t \in[\alpha, \beta]
$$

Então, como visto na Seção 1.1, uma solução de (1.16) é qualquer função $x:[\alpha, \beta] \rightarrow \mathcal{O}$ tal que

$$
x(v)-x(\gamma)=\int_{\gamma}^{v} D[F(x(\tau)) t]=\int_{\gamma}^{v} F(x(t)) d t
$$

para quaisquer $\gamma, v \in[\alpha, \beta]$, onde a última integral deve ser entendida no sentido da integral de Perron.

A partir daqui, vamos lidar com uma classe de problemas de valor inicial para EDOGs autônomas da forma

$$
\left\{\begin{array}{l}
\frac{d x}{d \tau}=D[F(x) t] \\
x(\alpha)=\tilde{x}
\end{array}\right.
$$

que admitem uma única solução $x:[\alpha, \beta] \rightarrow \mathcal{O}$, com $F: \mathcal{O} \rightarrow X$. De fato, como visto na Seção 1.3, temos a existência local e unicidade de uma solução sob determinadas condições, a saber, as condições (i), (ii) e (iii).

Denotamos por $I(\tilde{x})$ o intervalo maximal de existência de solução do problema (1.18). Diremos que a solução definida neste intervalo maximal de existência é a solução maximal do problema (1.18) e, de agora em diante, diremos que esta é a trajetória de $F$ através de $\tilde{x}$.

Dado $\tilde{x} \in \mathcal{O}$, seja $t \in I(\tilde{x})$ arbitrário. Diremos que o fluxo no tempo $t$ associado a $F: \mathcal{O} \rightarrow X$ em $\mathcal{O}$ é a aplicação $\phi^{t}: \mathcal{O} \rightarrow X$ que, a cada $\tilde{x} \in \mathcal{O}$, associa $\phi^{t}(\tilde{x})=x(t)$, onde $x: I(\tilde{x}) \rightarrow \mathcal{O}$ é a trajetória de $F$ através de $x(t)=\tilde{x}$. Isto quer dizer que, para cada $t$ variando em $I(\tilde{x})$, o fluxo determina a posição da solução com respeito à condição inicial $\tilde{x}$ no tempo $t$.

Para cada par $(t, \tilde{x})$ tal que $t \in I(\tilde{x})$ e $\tilde{x} \in \mathcal{O}$, definimos $\phi(t, \tilde{x})=x(t)$, onde $x: I(\tilde{x}) \rightarrow \mathcal{O}$ é a trajetória de $F$ através de $\tilde{x}$ e, então, obtemos uma aplicação

$$
\phi: \Omega \rightarrow X
$$

chamado fluxo de $F$ em $\mathcal{O}$. Aqui, o domínio $\Omega$ do fluxo é o conjunto de todos os pares $(t, \tilde{x}) \in I(\tilde{x}) \times \mathcal{O}$.

A partir de agora, vamos considerar que o intervalo maximal de solução do problema $(1.18)$ seja $I(\tilde{x})=[0,+\infty)$. 
Uma função $\tilde{x} \in \mathcal{O}$ será dita um ponto de equilíbrio ou singularidade de $F: \mathcal{O} \rightarrow X$, se

$$
F(\tilde{x})=0
$$

Note que um ponto de equilíbrio $\tilde{x}$ de $F$ é tal que $\phi(t, \tilde{x})=\tilde{x}$, para cada $t \in I(\tilde{x})$, ou seja, um ponto de equilíbrio de $F$ é um ponto fixo de seu fluxo associado.

Seja $\tilde{x}$ um ponto de equilíbrio de uma aplicação $F: \mathcal{O} \rightarrow X$, com $\mathcal{O} \subset X$ sendo um aberto. Diremos que $\tilde{x}$ é um ponto de equilíbrio estável de $F$, se para toda vizinhança (não necessariamente aberta) $U \subset X$ de $\tilde{x}$, existir uma vizinhança (não necessariamente aberta) $W \subset X$ de $\tilde{x}$ tal que $W \subset \mathcal{O} \cap U$ e

$$
\phi^{t}(y) \in U,
$$

para todo $y \in W$ e $t>0$.

A definição acima implica que um ponto de equilíbrio $\tilde{x}$ de $F$ é estável, se para todo $\epsilon>0$, pudermos encontrar um $\delta>0$ tal que $\left\|\phi_{t}(y)-\tilde{x}\right\| \leq \epsilon$ para cada $t>0$ e cada $y \in \mathcal{O} \subset X$, com $\|y-\tilde{x}\| \leq \delta$. Um ponto de equilíbrio de $F$ será dito instável, se não for estável.

Um ponto de equilíbrio $\tilde{x}$ de $F: \mathcal{O} \rightarrow X$ será dito assintoticamente estável, se for estável e tivermos

$$
\lim _{t \rightarrow+\infty} \phi^{t}(y)=\tilde{x},
$$

para qualquer $y \in W$, onde $W \subset X$ é a vizinhança da definição de ponto de equilíbrio estável.

Dado um ponto de equilíbrio assintoticamente estável $\tilde{x}$ de $F$, diremos que o conjunto $B(\tilde{x})=B_{F}(\tilde{x})$ dos pontos de $\mathcal{O}$ tais que as trajetórias por estes pontos tendem ao ponto de equilíbrio é a bacia de atração de $\tilde{x}$. Em outras palavras, $y \in \mathcal{O}$ pertencerá à bacia de atração $B(\tilde{x})$ de $\tilde{x}$ se, e somente se, tivermos

$$
\lim _{t \rightarrow+\infty} \phi^{t}(y)=\tilde{x} .
$$

Seja $F: \mathcal{O} \rightarrow X$ uma aplicação. Diremos que um conjunto $C \subset \mathcal{O}$ é positivamente invariante com respeito ao fluxo $\phi$ de $F$, se $\phi_{t}(C) \subset C$, para qualquer $t \geq 0$.

Proposição 1.15. Seja $\tilde{x}$ um ponto de equilíbrio assintoticamente estável de F. Então a bacia de atração $B(\tilde{x})$ definida acima é não vazia, aberta e positivamente invariante com respeito ao fluxo $\phi$ de $F$. 
Demonstração: Como $\tilde{x}$ é um ponto de equilíbrio assintoticamente estável de $F$, então $\tilde{x}$ é um ponto fixo do fluxo $\phi$ de $F$. Assim $\phi^{t}(\tilde{x})=\tilde{x}$ para qualquer $t \in[0,+\infty)$ e, então,

$$
\lim _{t \rightarrow+\infty} \phi^{t}(\tilde{x})=\tilde{x} .
$$

Logo $\tilde{x} \in B(\tilde{x})$, o que garante que a bacia de atração $B(\tilde{x})$ é não vazia.

Para facilitar nosso entendimento, vamos denotar por $x(t, \tilde{x})$, a solução $x$ no ponto $t$, com condição inicial $\tilde{x}$. Assim $x(0, \tilde{x})$ denota a solução $x$ no ponto 0 , com condição inicial $\tilde{x}$, que é precisamente $\tilde{x}$.

Observemos que, como $x$ é solução do problema (1.18), então $x(t+s, \tilde{x})$ é solução de (1.16), com condição inicial $x(0)=\tilde{x}$. Além disso, $x(t, x(s, \tilde{x}))$ é solução de $(1.16)$, com condição inicial $x(0)=x(s, \tilde{x})$. Pela existência e unicidade de soluções e do fato que, em $t=0$, temos

$$
x(0+s, \tilde{x})=x(s, \tilde{x})=x(0, x(s, \tilde{x})),
$$

então

$$
x(t+s, \tilde{x})=x(t, x(s, \tilde{x}))
$$

para quaisquer $t, s \in[0,+\infty)$. Esta propriedade é conhecida como a propriedade de grupo da solução. Assim,

$$
\phi^{t+s}(x)=\phi^{t}\left(\phi^{s}(x)\right)
$$

para quaisquer $t, s \in[0,+\infty)$ e qualquer $x \in \mathcal{O}$, e então

$$
\phi^{t+s}=\phi^{t} \circ \phi^{s}
$$

Agora, vamos usar a propriedade de grupo para mostrar a invariância da bacia de atração $B(\tilde{x})$. Seja $y \in B(\tilde{x})$. Então, por definição, $\lim _{t \rightarrow+\infty} \phi^{t}(y)=\tilde{x}$. Queremos mostrar que, dado $t \in[0,+\infty)$, temos

$$
\phi^{t}(y) \in B(\tilde{x})
$$

Mas note que

$$
\lim _{s \rightarrow+\infty} \phi^{s}\left(\phi^{t}(y)\right)=\lim _{s \rightarrow+\infty} \phi^{s+t}(y)=\tilde{x}
$$

onde a primeira igualdade vale pela propriedade de grupo e a última igualdade vale já que $t \in[0,+\infty)$ é dado e, então, $t+s \rightarrow+\infty$, quando $s \rightarrow+\infty$. Isto mostra a invariância positiva da bacia de atração sob a ação do fluxo. 
Finalmente, vamos mostrar que $B(\tilde{x})$ é um conjunto aberto. Note que as soluções do problema (1.18) são contínuas já que a solução da EDOG autônoma deve ser vista como solução da equação integral (1.17), e como pode ser visto em [25], Teoremas 1.14 e 1.16 , temos a continuidade da integral em (1.17), e portanto, a continuidade da solução. Assim, o fluxo $\phi$ e o fluxo no tempo $\phi^{t}$ também são contínuos.

Considere $z \in B(\tilde{x})$. Afirmamos que existe uma vizinhança de $z$ tal que todo ponto nesta vizinhança também tende a $\tilde{x}$. De fato, seja uma vizinhança $W_{0}$ de $\tilde{x}$ tal que $\lim _{s \rightarrow+\infty} \phi^{s}(y)=\tilde{x}$, para cada $y \in W_{0}$. Note que a existência da vizinhança $W_{0}$ está assegurada, pois $\tilde{x}$ é um ponto de equilíbrio assintoticamente estável. Como $z \in B(\tilde{x})$, então $y=\phi^{t_{0}}(z) \in W_{0}$ para algum $t_{0}$ suficientemente grande e, como $\phi^{t_{0}}(z)$ é contínuo em $z$, existe uma vizinhança $W$ de $z$ tal que $\phi^{t_{0}}(W) \subset W_{0}$.

Então, dado $w \in W$, temos

$$
\lim _{t \rightarrow+\infty} \phi^{t}(w)=\lim _{s \rightarrow+\infty} \phi^{s}\left(\phi^{t_{0}}(w)\right)=\tilde{x},
$$

pois $\phi^{t_{0}}(w) \in W_{0}$ e $s=t-t_{0} \rightarrow+\infty$, quando $t \rightarrow+\infty$. Isto significa que todo $w$ na vizinhança $W$ de $z$ tende a $\tilde{x}$ em tempo infinito e, assim, a bacia de atração é um conjunto aberto.

A seguir, definimos um conceito de funcional de Lyapunov para EDOGs autônomas.

Definição 1.16. Seja $x$ um ponto de equilíbrio de $F: \mathcal{O} \rightarrow X$. Diremos que uma função contínua $V: W \rightarrow \mathbb{R}$, com $W \subset X$ uma vizinhança de $x \in \mathcal{O}$, é um funcional de Lyapunov em $x$ com respeito a EDOG (1.16), se as seguintes condições forem satisfeitas:

- $V(x)=0$ e existe uma função $b: \mathbb{R}_{+} \rightarrow \mathbb{R}_{+}$, contínua e crescente, tal que $V(y) \geq b(\|y\|)$, para todo $y \in W \backslash\{x\}$

- Para cada solução $x:[\gamma, \nu] \rightarrow X$ de (1.16), onde $[\gamma, \nu] \subset[0,+\infty)$, vale

$$
\dot{V}(x(t))=\limsup _{\eta \rightarrow 0^{+}} \frac{V(x(t+\eta))-V(x(t))}{\eta} \leq 0,
$$

o que significa que a derivada à direita de $V$ ao longo de qualquer solução de (1.16) é não positiva.

O próximo teorema afirma que, se pudermos encontrar um funcional de Lyapunov em relação à equação (1.16) para um ponto de equilíbrio de $F$, então este ponto será um ponto de equilíbrio estável de $F$. 
Teorema 1.17. (Teorema de Lyapunov para EDOGs autônomas) Seja $x$ um ponto de equilíbrio de $F: \mathcal{O} \rightarrow X$. Se existir um funcional de Lyapunov com relação à equação (1.16), então $x$ será um ponto de equilíbrio estável de $F$.

Demonstração: Seja $V: W \rightarrow \mathbb{R}$ um funcional de Lyapunov em um ponto de equilíbrio $x$ de $F$, onde $W \subset X$ é uma vizinhança de $x \in \mathcal{O}$. Seja $U$ uma vizinhança qualquer de $x$ em $X$. Queremos mostrar que existe uma vizinhança $W_{0}$ de $x$ tal que $W_{0} \subset U$ e $\phi^{t}(y) \in U$, para quaisquer $y \in W_{0}$ e $t>0$. De fato, escolhemos $\delta>0$ tal que $B(x, \delta) \subset U \cap W$, onde $B(v, r)=\{u \in \mathcal{O} ;\|u-v\|<\delta\}$.

Note que

$$
\alpha=\inf _{\|y-x\|=\delta} V(y)>0
$$

já que $\delta>0$ está fixado e $V(y)>0$, para qualquer $y \in W \backslash\{x\}$.

Como o funcional $V$ é contínuo, então o conjunto $W_{0}=\{y \in B(x, \delta) ; V(y)<\alpha\}$ é aberto e, como $V(x)=0, W_{0}$ é uma vizinhança de $x$ e $W_{0} \subset B(x, \delta) \subset U \cap W$. Assim só nos resta mostrar que $\phi^{t}(y) \in U$, quaisquer que sejam $y \in W_{0}$ e $t>0$.

Dado $y \in W_{0}$, suponha que $\phi^{t}(y) \notin U$, para algum $t>0$. Então $\phi^{t}(y) \notin B(x, \delta)$ e, como $y \in W_{0} \subset B(x, \delta) \subset U \cap W$, em algum tempo $0<t_{*} \leq t$, a trajetória através de $y$ encontra $B(x, \delta)$, ou seja, $\left\|\phi_{t_{*}}-x\right\|=\delta$. Logo

$$
V\left(\phi^{0}(y)\right)=V(y)<\alpha \leq V\left(\phi^{t_{*}}(y)\right)
$$

o que contradiz a hipótese de que $V$ não cresce ao longo das trajetórias de $F$. Isto mostra que $\phi^{t}(y) \in U$, para quaisquer $y \in W_{0}$ e $t>0$, o que implica que $x$ é um ponto de equilíbrio estável.

O próximo teorema é o mais importante desta seção, já que ele relaciona funcionais de Lyapunov com o conceito de bacia de atração, mostrando, sob certas condições, que um ponto de equilíbrio $x$ de $F$ será assintoticamente estável e que um conjunto compacto e positivamente invariante pelo fluxo de $F: \mathcal{O} \rightarrow X$ estará contido na bacia de atração de $x$.

Teorema 1.18. Seja $x \in \mathcal{O}$ um ponto de equilíbrio de $F: \mathcal{O} \rightarrow X$ no conjunto aberto $\mathcal{O} \subset X$ e seja $C \subset \mathcal{O}$ um conjunto compacto que é positivamente invariante pelo fluxo de F. Seja $V: W \rightarrow \mathbb{R}$ um funcional de Lyapunov em relação à equação (1.16), onde $W$ vem da definição de funcional de Lyapunov, tal que $C \subset W$ e, para cada $y \neq x$ em $C$, existe um $t>0$ tal que $V\left(\phi^{t}(y)\right)<V(y)$, o que significa que não existe uma 
semi órbita regular positiva de $F$ na qual $V$ é constante. Então $x$ será assintoticamente estável e teremos $C \subset B(x)$.

Demonstração: Pelo Teorema 1.17, $x$ é um ponto de equilíbrio estável de $F$. Então é suficiente mostrarmos que $\phi_{t}(y) \rightarrow x$ para cada $y \in C$.

Suponha que $\lim _{t \rightarrow+\infty} \phi^{t}(y) \neq x$ para algum $y \in C$, isto é, existem $y \in C, \epsilon>0$ e uma sequência de tempos $t_{n} \rightarrow+\infty$ tais que $\left\|\phi^{t_{n}}(y)-x\right\| \geq \epsilon$, para cada $n \in \mathbb{N}$. Como $C$ é positivamente invariante, $\phi^{t_{n}}(y) \in C$, para todo $n$. Como $C$ é compacto e como toda sequência em um compacto possui uma subsequência convergente, podemos supor, sem perda de generalidade, que $\phi^{t_{n}}(y) \rightarrow a$, para algum $a \in C \backslash\{x\}$.

Por hipótese, $V$ não é constante ao longo da trajetória positiva $\phi^{t}(a)$. Assim, existe $t>0$ tal que $V\left(\phi^{t}(a)\right)<V(a)$. Tomemos $\alpha \in \mathbb{R}$ tal que $V\left(\phi^{t}(a)\right)<\alpha<V(a)$. Como o fluxo $\phi^{t}(z)$ é contínuo como função de $z$, com $t$ fixo, e pela continuidade de $V$, existe uma vizinhança $W_{a}$ de $a$ em $W$ tal que

$$
V\left(\phi^{t}(z)\right)<\alpha<V(z),
$$

para qualquer $z \in W_{a}$. Em particular, nenhum ponto de $W_{a}$ pode retornar para $W_{a}$ pelo fluxo de $F$, pois, uma vez deixado $W_{a}$ e, após um tempo $t$, o valor de $V$ nesta trajetória é sempre menor do que $\alpha$.

Como $\phi^{t_{n}}(y) \rightarrow a$, existe $t_{n_{0}}$ tal que $\phi^{t_{n_{0}}}(y) \in W_{a}$. Mas mostramos que $\phi^{t_{n}}(y)$ não pode retornar para $W_{a}$ com $t_{n}>t_{n_{0}}+t$, o que contradiz o fato que $\phi^{t_{n}}(y) \rightarrow a$ quando $n \rightarrow+\infty$. 


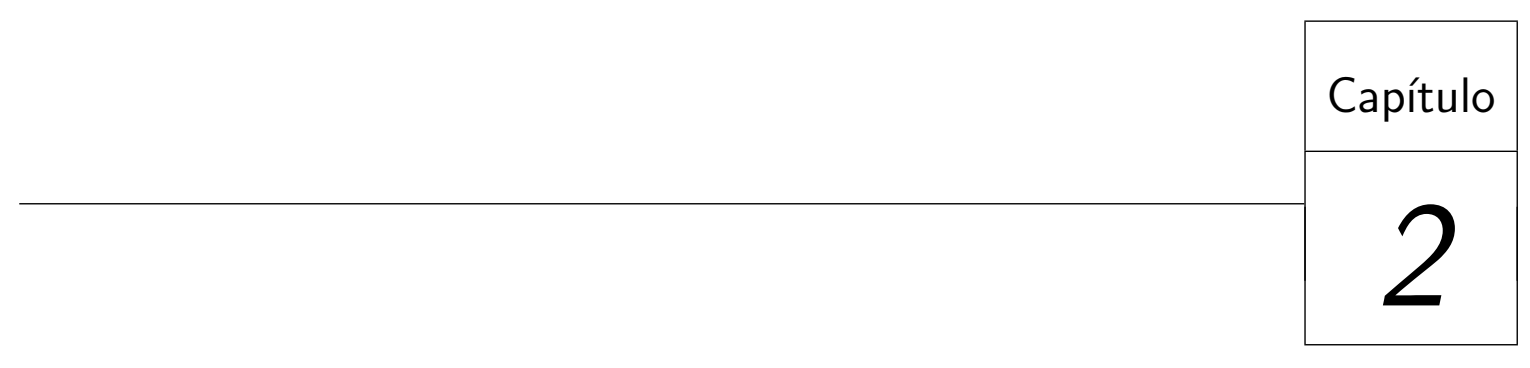

\section{Aplicações às EDOs autônomas}

Neste capítulo, aplicamos os resultados obtidos no Capítulo 1 a uma classe de EDOs autônomas. Antes disso, faremos algumas observações sobre a relação entre EDOGs autônomas e EDOs autônomas.

\subsection{EDOGs autônomas x EDOs autônomas}

Nesta seção, vamos mostrar uma nova abordagem para EDOs autônomas via EDOGs autônomas.

Relembramos ao leitor como uma EDOG se relaciona de maneira simples com uma EDO. Sabe-se que a EDO

$$
\frac{d x}{d t}=f(x, t), \quad t \in\left[t_{0}, T\right]
$$

tem forma integral dada por

$$
x(t)=x\left(t_{0}\right)+\int_{t_{0}}^{t} f(x(\tau), \tau) d \tau, \quad t \in\left[t_{0}, T\right],
$$


quando a integral existir em algum sentido. Sabe-se, também, que quando a integral em (2.2) existir no sentido de Riemann, Lebesgue (=McShane) ou Henstock-Kurzweil, então ela poderá ser aproximada por uma soma riemanniana da forma

$$
\sum_{i=1}^{m} f\left(x\left(\tau_{i}\right), \tau_{i}\right)\left[s_{i}-s_{i-1}\right]
$$

Alternativamente, se definirmos $F(z, s)=\int_{s_{0}}^{s} f(z, \sigma) d \sigma$, então a integral em $(2.2)$ poderá ser aproximada por

$$
\sum_{i=1}^{m} \int_{s_{i-1}}^{s_{i}} f\left(x\left(\tau_{i}\right), \sigma\right) d \sigma=\sum_{i=1}^{m}\left[F\left(x\left(\tau_{i}\right), s_{i}\right)-F\left(x\left(\tau_{i}\right), s_{i-1}\right)\right]
$$

onde $t_{0}=s_{0} \leq s_{1} \leq \ldots \leq s_{m}=t$ é uma partição finita do intervalo $\left[t_{0}, t\right]$ e, para cada $i, \tau_{i}$ está suficientemente "próximo" de $\left[s_{i-1}, s_{i}\right]$. Neste caso, o lado direito de (2.3) aproxima a integral de Kurzweil que, por sua vez, origina uma "equação diferencial" em um sentido mais amplo quando substituído em (2.2). Tal equação é conhecida como equação diferencial ordinária generalizada, a qual estamos nos referindo simplesmente como EDOG. Assim, uma relação biunívoca entre EDOs e EDOGs pode ser obtida.

A partir de agora, vamos descrever o contexto em que podemos relacionar uma EDO autônoma e uma EDOG autônoma.

Escreveremos $x \in G^{-}([a, b], X)$, sempre que $x \in G([a, b], X)$ e $x$ for uma função contínua à esquerda. Relembramos ao leitor que $G([a, b], X)$ denota o espaço das funções de $[a, b]$ em $X$ que são regradas. Este espaço, munido com a norma usual do supremo, é um espaço de Banach.

Sejam $t_{0}, \sigma \in \mathbb{R}$ com $\sigma>0$. Considere o seguinte problema de valor inicial para uma EDO autônoma

$$
\left\{\begin{array}{l}
\frac{d y}{d t}=f(y), \quad t \geq t_{0} \\
y\left(t_{0}\right)=y_{0}
\end{array}\right.
$$

onde $y_{0} \in \mathcal{O}, y:\left[t_{0}, t_{0}+\sigma\right] \rightarrow \mathcal{O}$ e $f: \mathcal{O} \rightarrow X$, onde $\mathcal{O} \subset X$ é um aberto.

A forma integral correspondente a (2.4) é dada por

$$
y(t)=y_{0}+\int_{t_{0}}^{t} f(y(s)) d s, \quad t \in\left[t_{0}, t_{0}+\sigma\right],
$$

onde vamos assumir integração no sentido de Perron. Então $y \in C\left(\left[t_{0}, t_{0}+\sigma\right], X\right)$, onde $C\left(\left[t_{0}, t_{0}+\sigma\right], X\right)$ denota o espaço de Banach das funções contínuas de $\left[t_{0}, t_{0}+\sigma\right]$ 
em $X$ com a norma do supremo. Denotaremos por $C\left(\left[t_{0}, t_{0}+\sigma\right], \mathcal{O}\right)$ o subconjunto de $C\left(\left[t_{0}, t_{0}+\sigma\right], X\right)$ das funções contínuas que assumem valores em $\mathcal{O} \subset X$, com $\mathcal{O}$ aberto.

A seguir, definimos solução do problema (2.4).

Definição 2.1. Considere o problema (2.4), onde $f: \mathcal{O} \rightarrow X$, e para todo y $\in$ $C\left(\left[t_{0}, t_{0}+\sigma\right], \mathcal{O}\right)$, a aplicação $t \mapsto f(y(t))$ é Perron integrável em $\left[t_{0}, t_{0}+\sigma\right]$. Se existir uma função $y \in C\left(\left[t_{0}, t_{0}+\sigma\right], \mathcal{O}\right)$ tal que valem as seguintes condições

- $\dot{y}(t)=f(y(t))$, para quase todo $t \in\left[t_{0}, t_{0}+\sigma\right]$

- $y\left(t_{0}\right)=y_{0}$,

então diremos que y é uma solução de (2.4) em $\left[t_{0}, t_{0}+\sigma\right]$, com condição inicial $\left(y_{0}, t_{0}\right)$.

Consideremos o problema (2.4), onde $y_{0} \in \mathcal{O}, f$ aplica $\mathcal{O}$ em $X$ e, para todo $y \in C\left(\left[t_{0}, t_{0}+\sigma\right], \mathcal{O}\right)$, a aplicação $t \mapsto f(y(t))$ é uma função Perron integrável em $\left[t_{0}, t_{0}+\sigma\right]$. Suponha que as seguintes condições estejam satisfeitas:

(M) Existe uma função Lebesgue integrável $M:\left[t_{0}, t_{0}+\sigma\right] \rightarrow \mathbb{R}$ tal que, para qualquer $z \in C\left(\left[t_{0}, t_{0}+\sigma\right], \mathcal{O}\right)$ e quaisquer $s_{1}, s_{2} \in\left[t_{0}, t_{0}+\sigma\right]$

$$
\left\|\int_{s_{1}}^{s_{2}} f(z(s)) d s\right\| \leq \int_{s_{1}}^{s_{2}} M(s) d s
$$

(L) Existe uma função Lebesgue integrável $L:\left[t_{0}, t_{0}+\sigma\right] \rightarrow \mathbb{R}$ tal que, para quaisquer $z, w \in C\left(\left[t_{0}, t_{0}+\sigma\right], \mathcal{O}\right)$ e quaisquer $s_{1}, s_{2} \in\left[t_{0}, t_{0}+\sigma\right]$,

$$
\left\|\int_{s_{1}}^{s_{2}}[f(z(s))-f(w(s))] d s\right\| \leq\|z-w\|_{\infty} \int_{s_{1}}^{s_{2}} L(s) d s .
$$

Agora, para cada $z \in \mathcal{O}$ e cada $t \in\left[t_{0}, t_{0}+\sigma\right]$, seja $G: \mathcal{O} \times\left[t_{0}, t_{0}+\sigma\right] \rightarrow \mathbb{R}^{n}$ definida por

$$
G(z, t)=f(z) t
$$

Seja $F: \mathcal{O} \rightarrow X$ definida por $F(z)=f(z)$, ou seja, $F=f$. Então $G(z, t)=F(z) t$.

Considere o problema de valor inicial para a EDOG autônoma

$$
\left\{\begin{array}{l}
\frac{d x}{d \tau}=D G(x, t)=D[F(x) t] \\
x\left(t_{0}\right)=y_{0}
\end{array}\right.
$$


onde $F=f$. Então uma função $x$ definida no intervalo $\left[t_{0}, t_{0}+\sigma\right]$ e tomando valores em $\mathcal{O}$ será uma solução do problema (2.7) no intervalo $\left[t_{0}, t_{0}+\sigma\right]$, se

$$
x(t)=y_{0}+\int_{t_{0}}^{t} F(x(s)) d s, \quad t \in\left[t_{0}, t_{0}+\sigma\right] .
$$

Note que as equações (2.5) e (2.8) coincidem, ou seja, uma solução $y$ da EDO autônoma (2.4) pode ser escrita como uma solução $x$ da EDOG autônoma (2.7), isto é, $y \equiv x$ em $\left[t_{0}, t_{0}+\sigma\right]$.

Note, também, que nas condições $(\mathrm{M})$ e $(\mathrm{L})$ consideradas há pouco, temos $G \in$ $\widetilde{\mathcal{F}}(\Omega, h) \operatorname{com} \Omega=\mathcal{O} \times\left[t_{0}, t_{0}+\sigma\right]$ e $h:\left[t_{0}, t_{0}+\sigma\right] \rightarrow \mathbb{R}$ dada por

$$
h(t)=\int_{t_{0}}^{t}[M(s)+L(s)] \mathrm{d} s, \quad t \in\left[t_{0}, t_{0}+\sigma\right] .
$$

De fato, pois nas condições (M) e (L) temos

$$
\left\|\int_{s_{1}}^{s_{2}} F(z(s)) \mathrm{d} s\right\| \leq \int_{s_{1}}^{s_{2}} M(s) d s \leq\left|h\left(s_{2}\right)-h\left(s_{1}\right)\right|
$$

e

$$
\left\|\int_{s_{1}}^{s_{2}}[F(z(s))-F(w(s))] \mathrm{d} s\right\| \leq\|z-w\|_{\infty} \int_{s_{1}}^{s_{2}} L(s) d s \leq\|z-w\|_{\infty}\left|h\left(s_{2}\right)-h\left(s_{1}\right)\right|
$$

para quaisquer $s_{1}, s_{2} \in\left[t_{0}, t_{0}+\sigma\right]$ e quaisquer $z, w \in C\left(\left[t_{0}, t_{0}+\sigma\right], \mathcal{O}\right)$.

Assim, a partir do teorema de existência-unicidade para EDOGs autônomas (Teorema 1.11), fica evidente o teorema seguinte.

Teorema 2.2. Considere a EDO autônoma (2.4) e suponha que as condições (M) e (L) estejam satisfeitas. Então o problema (2.4) admite uma única solução (local).

Demonstração: Pelo que fizemos acima e pelo Teorema 1.11, temos a existência de uma única solução para a EDOG autônoma (2.7) e, portanto, o mesmo se aplica para o sistema (2.4), sob as condições (M) e (L). 


\subsection{Dependência contínua de soluções com respeito às condições iniciais}

Nesta seção, mostraremos um teorema sobre dependência contínua de soluções com respeito às condições iniciais para EDOs autônomas. Esta é uma aplicação dos resultados obtidos no capítulo anterior e pode ser encontrada em [15].

Sejam $t_{0}, \sigma \in \mathbb{R}$, com $\sigma>0$. Vamos considerar a seguinte sequência de problemas de valor inicial para EDOs autônomas

$$
\left\{\begin{array}{l}
\frac{d y}{d t}=f_{p}(y), \quad t \in\left[t_{0}, t_{0}+\sigma\right] \\
y\left(t_{0}\right)=y_{0}^{p}
\end{array}\right.
$$

onde, para cada $p=0,1,2, \ldots, y_{0}^{p} \in \mathcal{O}$ e $f_{p}$ aplica $\mathcal{O}$ em $X$.

Sabe-se que, para todo $p=0,1,2, \ldots$, o sistema (2.10) tem forma integral dada por

$$
y(t)=y_{0}^{p}+\int_{t_{0}}^{t} f_{p}(y(s)) d s, \quad t \in\left[t_{0}, t_{0}+\sigma\right] .
$$

Vamos assumir que, para cada $p=0,1, \ldots$, a aplicação $t \mapsto f_{p}(y(t))$ seja Perron integrável em $\left[t_{0}, t_{0}+\sigma\right]$ para todo $y \in C\left(\left[t_{0}, t_{0}+\sigma\right], \mathcal{O}\right)$ e que as funções $f_{p}$ satisfaçam as condições $(\mathrm{M})$ e $(\mathrm{L})$ da seção anterior com as mesmas funções $M$ e $L$, independente de $p$.

Definimos, para cada $p=0,1, \ldots$ e para $x \in \mathcal{O}$, as funções $F_{p}(x)=f_{p}(x)$. Pela seção anterior, as funções $G_{p}: \mathcal{O} \times\left[t_{0}, t_{0}+\sigma\right] \rightarrow X, p=0,1, \ldots$, dadas por

$$
G_{p}(x, t)=F_{p}(x) t
$$

pertencem à mesma classe $\widetilde{\mathcal{F}}(\Omega, h)$, onde $\Omega=\mathcal{O} \times\left[t_{0}, t_{0}+\sigma\right]$ e $h:\left[t_{0}, t_{0}+\sigma\right] \rightarrow \mathbb{R}$ é dada por

$$
h(t)=\int_{t_{0}}^{t}[M(s)+L(s)] d s, \quad t \in\left[t_{0}, t_{0}+\sigma\right] .
$$

Então o problema (2.10) pode ser reescrito como uma EDOG autônoma

$$
\left\{\begin{array}{l}
\frac{d x}{d \tau}=D\left[F_{p}(x) t\right] \\
x_{p}\left(t_{0}\right)=y_{0}^{p}
\end{array}\right.
$$


Daí, utilizando a Proposição 1.14, é fácil enunciar e demonstrar um teorema sobre dependência contínua de soluções, com respeito às condições iniciais, para EDOs autônomas do tipo (2.10), como corolário do Teorema 4.1 de [12].

Teorema 2.3. Suponha que, para cada $p=0,1, \ldots$, tenhamos $y_{0}^{p} \in \mathcal{O}$ e que, para cada $y \in C\left(\left[t_{0}, t_{0}+\sigma\right], \mathcal{O}\right), t \mapsto f_{p}(y(t))$ seja Perron integrável em $\left[t_{0}, t_{0}+\sigma\right]$. Suponha, também, que as funções $f_{p}$ satisfaçam as condições $(M)$ e $(L)$ da seção anterior para as mesmas funções $M$ e $L$ para $p=0,1, \ldots$ Suponha, ainda, que

$$
\lim _{p \rightarrow \infty} \sup _{\vartheta \in\left[t_{0}, t_{0}+\sigma\right]}\left\|\int_{t_{0}}^{\vartheta}\left[f_{p}(y(s))-f_{0}(y(s))\right] d s\right\|=0, \quad y \in C\left(\left[t_{0}, t_{0}+\sigma\right], X\right),
$$

e que, para cada $p=1,2, \ldots, y_{p}:\left[t_{0}, t_{0}+\sigma\right] \rightarrow \mathcal{O}$ seja uma solução do problema $(2.10)$ em $\left[t_{0}, t_{0}+\sigma\right]$, com

$$
\lim _{p \rightarrow \infty} y_{p}(s)=y(s) e m\left[t_{0}, t_{0}+\sigma\right]
$$

e $y(s) \in \mathcal{O}$, para todo $s \in\left[t_{0}, t_{0}+\sigma\right]$. Então $y:\left[t_{0}, t_{0}+\sigma\right] \rightarrow \mathcal{O}$ será uma solução do problema

$$
\left\{\begin{array}{l}
\frac{d y}{d t}=f_{0}(y), \quad t \in\left[t_{0}, t_{0}+\sigma\right] \\
y\left(t_{0}\right)=y_{0}
\end{array}\right.
$$

Demonstração: Por hipótese, para cada $p=1,2, \ldots, y_{p}$ é uma solução em $\left[t_{0}, t_{0}+\sigma\right]$ de (2.10) que é equivalente a (2.13). Segue da Proposição 1.14 o fato de que

$$
\lim _{p \rightarrow \infty} y_{p}(s)=y(s), \text { uniformemente em }\left[t_{0}, t_{0}+\sigma\right]
$$

e que $y:\left[t_{0}, t_{0}+\sigma\right] \rightarrow \mathcal{O}$ é uma solução do problema

$$
\left\{\begin{array}{l}
\frac{d x}{d \tau}=D\left[F_{0}(x) t\right], \quad t \in\left[t_{0}, t_{0}+\sigma\right] \\
x\left(t_{0}\right)=y_{0}
\end{array}\right.
$$

ou seja, a função $y:\left[t_{0}, t_{0}+\sigma\right] \rightarrow \mathcal{O}$ é o limite uniforme das funções $y_{p}$ e $y$ é uma solução do problema (2.16), o que conclui a demonstração.

Uma aplicação imediata do Teorema 2.3 é similar a que foi descrita na Introdução. Seja $g_{0}:[\alpha, \beta] \rightarrow \mathbb{R}^{n}$, uma função contínua que não é diferenciável em qualquer ponto de seu domínio. Seja $g_{k}:[\alpha, \beta] \rightarrow \mathbb{R}^{n}, k=1,2, \ldots$, uma sequência de funções em $C^{1}\left([\alpha, \beta], \mathbb{R}^{n}\right)$, onde $C^{1}\left([\alpha, \beta], \mathbb{R}^{n}\right)$ denota o subconjunto de $C\left([\alpha, \beta], \mathbb{R}^{n}\right)$ das funções que são continuamente diferenciáveis em todo ponto de $[\alpha, \beta]$. Suponhamos que as 
funções $g_{k}$ satisfaçam as condições (M) e (L) da Seção 2.1, com as mesmas funções $M$ e $L$, para todo $k=1,2, \ldots$. Assim $g_{k} \in \widetilde{\mathcal{F}}(\Omega, h), k=1,2, \ldots, \operatorname{com} \Omega=\mathbb{R}^{n} \times[\alpha, \beta]$ e $h:[\alpha, \beta] \rightarrow \mathbb{R}$ dada por

$$
h(t)=\int_{\alpha}^{t}[M(s)+L(s)] d s, \quad t \in[\alpha, \beta] .
$$

Consideramos em $\widetilde{\mathcal{F}}(\Omega, h)$ a topologia métrica caracterizada pela convergência

$$
g_{k} \rightarrow g_{0} \quad \text { se } \quad \int_{\alpha}^{\beta} g_{k}(s) d s \rightarrow \int_{\alpha}^{\beta} g_{0}(s) d s
$$

Se $g_{k}^{\prime}$ for a derivada de $g_{k}$ para $k=1,2, \ldots$, então a sequência $\left\{g_{k}^{\prime}\right\}$ é de Cauchy, mas não possui um limite sendo o lado direito de uma EDO. A solução da equação associada à $g_{k}^{\prime}$ é, a menos de uma constante, sua primitiva, $g_{k}$, e assim a solução limite $g_{0}$ não é uma solução de uma EDO. Este problema não ocorre quando tratamos tais equações vistas como EDOGs, já que, no caso de EDOGs, a equação é definida em termos da forma integral via integral de Kurzweil.

\subsection{Bacia de atração para EDOs autônomas}

Considere o seguinte problema de valor inicial para uma EDO autônoma

$$
\left\{\begin{array}{l}
\dot{y}(t)=f(y), \quad t \in\left[t_{0},+\infty\right) \\
y\left(t_{0}\right)=y_{0}
\end{array}\right.
$$

onde $y_{0} \in \mathcal{O}, y:\left[t_{0},+\infty\right) \rightarrow \mathcal{O}$ e $f$ aplica $\mathcal{O}$ em $X$.

Como visto na Seção 2.1, relacionamos EDOGs autônomas com EDOs autônomas renomeando $F=f$. Assim, podemos definir todos os conceitos de bacia de atração para EDOs da mesma forma que definimos tais conceitos para EDOGs. Os resultados podem ser transferidos imediatamente para EDOs.

Proposição 2.4. Seja y um ponto de equilíbrio assintoticamente estável de $f$. A bacia de atração, $B(y)$, é não-vazia, aberta em $\mathcal{O} \subset X$ e invariante com respeito ao fluxo no tempo $\phi^{t}$ de $f$.

Claramente, se $x \in \mathcal{O} \subset X$ for um ponto de equilíbrio estável para a EDOG (1.18), com $F=f$, então $x$ será ponto de equilíbrio estável para a EDO autônoma (2.20). 
Considere também a EDO autônoma

$$
\frac{d y}{d t}=f(y(t))
$$

onde $f$ aplica $\mathcal{O}$ em $X$.

Definição 2.5. Seja y um ponto de equilíbrio de $f$. Diremos que uma função contínua $V: W \rightarrow \mathbb{R}$, com $W \subset X$ uma vizinhança de $y$, é um funcional de Lyapunov (com respeito à EDO (2.21)) em y, se as seguintes condições forem satisfeitas:

- $V(y)=0$, e existe uma função $b: \mathbb{R}_{+} \rightarrow \mathbb{R}_{+}$, contínua e crescente, tal que $V(z) \geq b(\|z\|)$, para cada $z \in W \backslash\{y\}$

- Para cada solução y : $\left[t_{0},+\infty\right) \rightarrow X$ de $(2.21)$,

$$
\dot{V}(y)=\limsup _{\eta \rightarrow 0^{+}} \frac{V(y(t+\eta))-V(y(t))}{\eta} \leq 0,
$$

ou seja, a derivada à direita de $V$ é não-positiva ao longo de toda solução de $(2.21)$.

Claramente, se $V: W \rightarrow \mathbb{R}$ for um funcional de Lyapunov para o ponto de equilíbrio $y \in X$, então $V$ também será um funcional de Lyapunov para $y$, solução da EDOG

$$
\frac{d x}{d \tau}=D[F(x) t]
$$

A recíproca também é verdadeira e imediata e vale o resultado seguinte.

Teorema 2.6. (Teorema de Lyapunov para EDOs autônomas) Seja y um ponto de equilíbrio de $f: M_{1} \rightarrow X$, onde $M_{1} \subset X$ é um conjunto aberto. Se existir um funcional de Lyapunov para $f$ em $y$, então y será um ponto de equilíbrio estável de $f$.

Demonstração: Dado um funcional de Lyapunov para a EDO autônoma (2.21), obtemos um funcional de Lyapunov para EDOG autônoma com $F=f$. Pelo Teorema 1.17 para o caso de uma EDOG autônoma, o ponto de equilíbrio $x$ de $F$ é estável e pelas observações feitas acima, $x$ é um ponto de equilíbrio estável de $f$.

Também temos o resultado abaixo.

Teorema 2.7. Seja $y \in M_{1}$ um ponto de equilíbrio de $f$ no conjunto aberto $M_{1} \subset X$ e seja $C \subset M_{1}$ um conjunto compacto que é positivamente invariante pelo fluxo de $f$. 
Seja $V: W \rightarrow \mathbb{R}$ um funcional de Lyapunov em relação à equação (2.21), onde $W$ vem da definição de funcional de Lyapunov, tal que $C \subset W$ e, para cada $z \neq y$ em $C$, existe $t>0$ tal que $V\left(\phi^{t}(z)\right)<V(z)$, ou seja, não existe uma semi-órbita positiva de f na qual $V$ é constante. Então y será assintoticamente estável $e C \subset B(y)$.

Demonstração: A demonstração deste teorema é simples, se observarmos novamente que, dado um funcional de Lyapunov para a EDO autônoma (2.21), obtemos um funcional de Lyapunov para EDOG autônoma com $F=f$ e, portanto, a demonstração segue como na demonstração do Teorema 1.18 . 



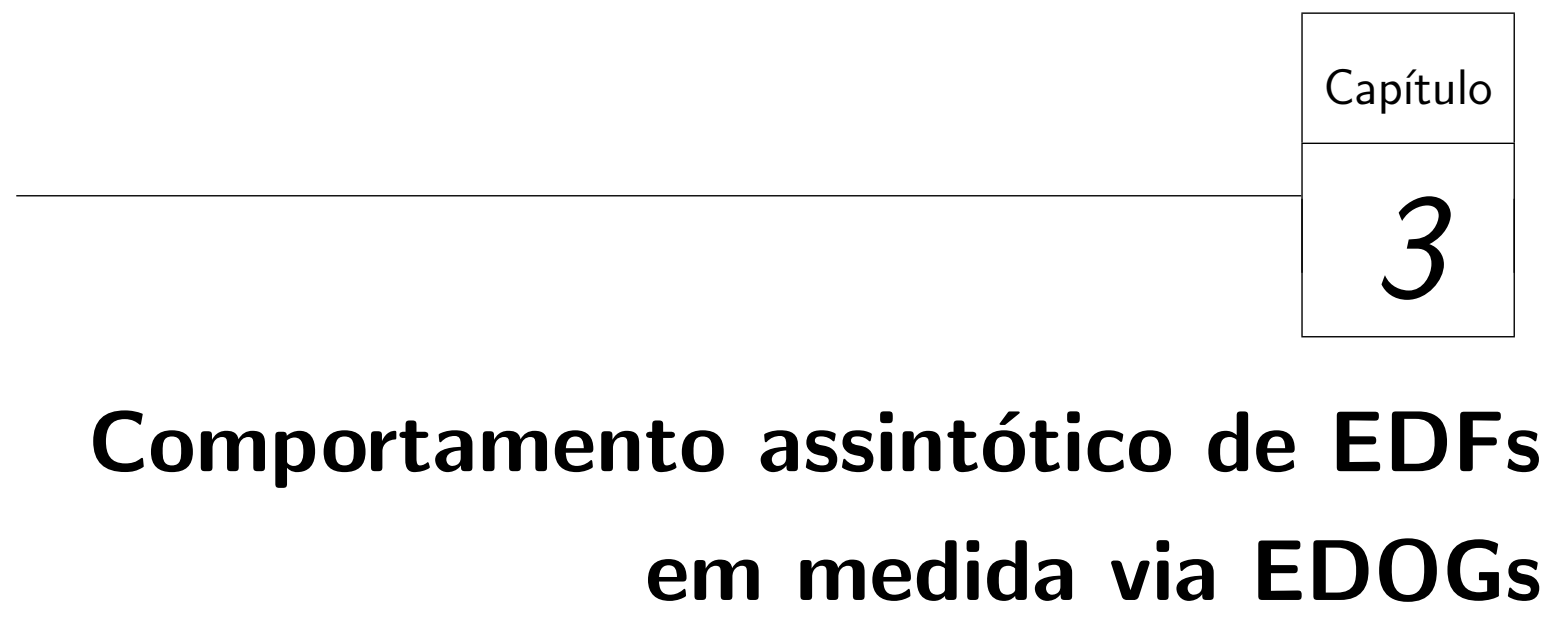

Neste capítulo, faremos uma breve apresentação de como é feita a relação entre EDOGs e equações diferenciais funcionais em medida e apresentaremos novos resultados de estabilidade para tais equações via EDOGs. Vamos definir equações diferenciais funcionais em medida e apresentar alguns novos conceitos de estabilidade para tais equações.

Em [10], os autores introduzem o conceito de equações diferenciais chamadas equações diferenciais funcionais em medida (escrevemos EDFs em medida) que podem ser descritas pela seguinte equação:

$$
D x=f\left(x_{t}, t\right) D g,
$$

onde $x_{t}$ é dado pela fórmula $x_{t}(\theta)=x(t+\theta), \theta \in[-r, 0]$, com $r>0, f: G\left([-r, 0], \mathbb{R}^{n}\right) \times$ $\left[t_{0},+\infty\right) \rightarrow \mathbb{R}^{n}$, com $t_{0} \geq 0, D x$ e $D g$ são as derivadas distribuicionais, com respeito a $x$ e $g$, no sentido das distribuições de L. Schwartz (veja [8]).

No caso em que $g(t)=t$, a equação (3.1) se reduz a uma equação diferencial funcional com retardamento no sentido usual. 
A forma integral equivalente a (3.1) é dada por

$$
x(t)=x\left(t_{0}\right)+\int_{t_{0}}^{t} f\left(x_{s}, s\right) d g(s), \quad t \in\left[t_{0},+\infty\right),
$$

onde vamos considerar a integral de Kurzweil-Henstock-Stieltjes tomada com respeito à função $g:\left[t_{0},+\infty\right) \rightarrow \mathbb{R}$ que consideraremos não decrescente.

Como estamos interessados em estabelecer uma teoria de estabilidade para a solução trivial de (3.1), consideramos, daqui para frente, que $f(0, t)=0$ para todo $t \in\left[t_{0}, \infty\right)$ o que garante que $y \equiv 0$ é uma solução de $(3.1)$ em $\left[t_{0}-r, \infty\right)$.

Antes de apresentarmos os novos e os já conhecidos conceitos de estabilidade para a solução trivial de (3.1), vamos expor alguns fundamentos da teoria de integração de Kurzweil-Henstock-Stieltjes.

\subsection{Integração de Kurzweil-Henstock-Stieltjes}

Vamos introduzir algumas propriedades básicas da integral de Kurzweil-HenstockStieltjes. Lembramos que a definição desta integral foi mencionada no início do Capítulo 1, como caso particular da integral de Kurzweil $\int_{\alpha}^{\beta} D U(\tau, t)$, quando $U:[a, b] \times[a, b] \rightarrow$ $X$ é dado por $U(\tau, t)=f(\tau) g(t)$. Neste caso, temos a integral de Perron-Stieltjes, também conhecida como integral de Kurzweil-Henstock-Stieltjes sobre $[\alpha, \beta]$ que denotaremos simplesmente por $\int_{a}^{b} f(t) d g(t)$.

A seguir, vamos enunciar dois resultados que tratam da existência da integral de Kurzweil-Henstock-Stieltjes $\int_{a}^{b} f(t) d g(t)$.

Uma demonstração do próximo resultado pode ser encontrada em [25], Corolário 1.34; a desigualdade segue diretamente da definição da integral de Kurzweil-HenstockStieltjes.

Denotaremos por $|\cdot|$ qualquer norma de $\mathbb{R}^{n}$.

Teorema 3.1. Se $f:[a, b] \rightarrow \mathbb{R}^{n}$ for uma função regrada e $g:[a, b] \rightarrow \mathbb{R}$ for uma função não decrescente, então a integral de Kurzweil-Henstock-Stieltjes $\int_{a}^{b} f(t) d g(t)$ existirá. Além disso, se $|f(s)| \leq C$ para todo $s \in[a, b]$, então

$$
\left|\int_{a}^{b} f(s) d g(s)\right| \leq C[g(b)-g(a)] .
$$

Lembramos que, quando consideramos $g$ uma função não decrescente, então $g$ pode conter infinitos pontos de descontinuidade. 
O resultado a seguir, que descreve as propriedades da integral indefinida de KurzweilHenstock-Stieltjes, é um caso especial do Teorema 1.16 de [25].

Teorema 3.2. Sejam $f:[a, b] \rightarrow \mathbb{R}^{n}$ e $g:[a, b] \rightarrow \mathbb{R}$ um par de funções tais que $g$ é regrada e a integral de Kurzweil-Henstock-Stieltjes $\int_{a}^{b} f(t) d g(t)$ existe. Então a função

$$
h(t)=\int_{a}^{t} f(s) d g(s), \quad t \in[a, b]
$$

é regrada e satisfaz

$$
\begin{aligned}
h(t+) & =h(t)+f(t) \Delta^{+} g(t), \quad t \in[a, b), \\
e & \\
h(t-) & =h(t)-f(t) \Delta^{-} g(t), \quad t \in(a, b],
\end{aligned}
$$

onde $\Delta^{+} g(t)=g(t+)-g(t) e \Delta^{-} g(t)=g(t)-g(t-)$.

Em várias partes deste trabalho, vamos precisar da extensão da integral de KurzweilHenstock-Stieltjes sobre intervalos ilimitados da reta $\mathbb{R}$.

Para podermos estender a definição da integral de Kurzweil-Henstock-Stieltjes para intervalos não limitados $(-\infty, c]$ ou $[c,+\infty)$, é necessário definir $\delta$-vizinhanças de $-\infty$ e de $+\infty$. Para isto, toma-se $\delta(-\infty)$ e $\delta(\infty)$ positivos e arbitrários e considera-se as vizinhanças

$$
\left[-\infty,-\frac{1}{\delta(-\infty)}\right) \text { e }\left(\frac{1}{\delta(\infty)}, \infty\right]
$$

Desta forma, $-\infty$ e $\infty$ são necessariamente as marcas em relação a qualquer calibre definido na reta estendida $\overline{\mathbb{R}}$.

Quando queremos definir $\int_{-\infty}^{+\infty} f(t) d g(t)$, tomamos $f(-\infty)=f(\infty)=0$ e assim dada uma divisão marcada $\delta$-fina da reta estendida, na soma de Riemann

$$
\sum_{i=1}^{k(d)} f\left(\tau_{i}\right)\left[g\left(t_{i}\right)-g\left(t_{i-1}\right)\right]
$$

o primeiro e o último termos da soma são nulos, pois $\tau_{1}=t_{0}=-\infty$ e $\tau_{k(d)}=+\infty$. Aqui convencionamos que $0 . \infty=0$. Para mais detalhes sobre a extensão da integral de Kurzweil a $\overline{\mathbb{R}}$, o leitor pode consultar a Seção 7 de [20]. 


\subsection{Novos conceitos de estabilidade para EDFs em me- dida}

Vamos dizer que um conjunto $O \subset G\left(\left[t_{0}-r,+\infty\right), \mathbb{R}^{n}\right)$ tem a propriedade do prolongamento, se para todo $y \in O$ e todo $\bar{t} \in\left[t_{0}-r,+\infty\right)$, a função $\bar{y}$ dada por

$$
\bar{y}(t)= \begin{cases}y(t), & t_{0}-r \leq t \leq \bar{t} \\ y(\bar{t}), & \bar{t}<t<\infty\end{cases}
$$

também for um elemento de $O$.

Seja $S=\left\{y_{t} ; y \in O, t \in\left[t_{0}, \infty\right)\right\}$. Então $S \subset G\left([-r, 0], \mathbb{R}^{n}\right)$.

Seja $g:\left[t_{0},+\infty\right) \rightarrow \mathbb{R}$ não decrescente e considere as seguintes condições sobre a função $f: S \times\left[t_{0},+\infty\right) \rightarrow \mathbb{R}^{n}$ :

$\left(H_{1}\right)$ A integral de Kurzweil-Henstock-Stieltjes $\int_{t_{0}}^{t} f\left(y_{s}, s\right) d g(s)$ existe para quaisquer $y \in O$ e $t \in\left[t_{0}, \infty\right)$.

$\left(H_{2}\right)$ Existe uma função $M:\left[t_{0}, \infty\right) \rightarrow \mathbb{R}$ localmente Lebesgue-Stieltjes integrável com respeito a $g$ tal que a desigualdade

$$
\left|\int_{u}^{v} f\left(y_{s}, s\right) d g(s)\right| \leq \int_{u}^{v} M(s) d g(s)
$$

vale para quaisquer $y \in O$ e $u, v \in\left[t_{0}, \infty\right)$.

$\left(H_{3}\right)$ Existe uma função $L:\left[t_{0}, \infty\right) \rightarrow \mathbb{R}$ localmente Lebesgue-Stieltjes integrável com respeito a $g$ tal que a desigualdade

$$
\left|\int_{u}^{v}\left[f\left(y_{s}, s\right)-f\left(z_{s}, s\right)\right] d g(s)\right| \leq \int_{u}^{v} L(s)\left\|y_{s}-z_{s}\right\|_{\infty} d g(s)
$$

vale para quaisquer $y, z \in O$ e $u, v \in\left[t_{0}, \infty\right)$.

Lembramos ao leitor que consideramos $f(0, t)=0$ para todo $t \in\left[t_{0}, \infty\right)$ e portanto $y \equiv 0$ é uma solução de $(3.1)$ em $\left[t_{0}-r, \infty\right)$.

Introduzimos, primeiramente, algumas noções básicas de estabilidade para a solução $y \equiv 0$ de (3.1). As seguintes três definições são o que chamaríamos de "definições clássicas" de estabilidade de Lyapunov, estabilidade (de Lyapunov) uniforme e estabilidade uniforme assintótica da solução trivial da EDF em medida (3.1). Estas definições po- 
dem ser encontradas em [17], por exemplo, para o caso de EDFs com retardamento que são um caso particular de EDFs em medida.

Definição 3.3. A solução trivial da equação (3.1) será dita estável no sentido de Lyapunov, se para todo $\varepsilon>0$ e todo $\gamma \in \mathbb{R}, \gamma \geq t_{0}$, existir $\delta=\delta(\varepsilon, \gamma)>0$ tal que, se $\phi \in S$ e $\bar{y}:[\gamma, v] \rightarrow \mathbb{R}^{n}, \operatorname{com}[\gamma, v] \subset\left[t_{0},+\infty\right)$, for uma solução de (3.1) tal que $\bar{y}_{\gamma}=\phi$ e

$$
\|\phi\|_{\infty}<\delta
$$

então

$$
\left\|\bar{y}_{t}(\gamma, \phi)\right\|_{\infty}<\varepsilon, \quad t \in[\gamma, v] .
$$

Definição 3.4. A solução trivial do sistema (3.1) será dita uniformemente estável, se o número $\delta$ na Definição 3.3 for independente de $\gamma$.

Definição 3.5. A solução $y \equiv 0$ de (3.1) será dita uniformemente assintoticamente estável, se existir um $\delta_{0}>0$ e para todo $\varepsilon>0$, existir um $T=T\left(\varepsilon, \delta_{0}\right) \geq 0$ tal que se $\phi \in S$, e $\bar{y}:[\gamma, v] \rightarrow \mathbb{R}^{n}, \operatorname{com}[\gamma, v] \subset\left[t_{0},+\infty\right)$, for solução de (3.1) tal que $\bar{y}_{\gamma}=\phi e$

$$
\|\phi\|_{\infty}<\delta_{0}
$$

então

$$
\left\|\bar{y}_{t}(\gamma, \phi)\right\|_{\infty}<\varepsilon, \quad t \in[\gamma, v] \cap[\gamma+T,+\infty) .
$$

Considere a seguinte EDF em medida com perturbação

$$
D y=f\left(y_{t}, t\right) D g+p(t) D u \text {. }
$$

Vamos considerar que as funções $g, u:\left[t_{0},+\infty\right) \rightarrow \mathbb{R}$ sejam não decrescentes e, portanto, podem conter infinitos pontos de descontinuidade.

A forma integral de (3.2) é dada por

$$
y(t)=y\left(t_{0}\right)+\int_{t_{0}}^{t} f\left(y_{s}, s\right) d g(s)+\int_{t_{0}}^{t} p(s) d u(s), \quad t \in\left[t_{0}, \infty\right),
$$

onde as integrais são consideradas no sentido de Kurzweil-Henstock-Stieltjes. Uma solução de (3.2), quando existir, será regrada (veja [10]).

Assumimos as condições $\left(H_{1}\right),\left(H_{2}\right)$ e $\left(H_{3}\right)$ sobre $f: S \times\left[t_{0},+\infty\right) \rightarrow \mathbb{R}^{n}$ e também as seguintes condições sobre a função $p:\left[t_{0}, \infty\right) \rightarrow \mathbb{R}^{n}$ :

$\left(H_{4}\right)$ A integral de Kurzweil-Henstock-Stieltjes $\int_{t_{0}}^{t} p(s) d u(s)$ existe para qualquer $t \in$ $\left[t_{0}, \infty\right)$; 
$\left(H_{5}\right)$ Existe uma função $K:\left[t_{0}, \infty\right) \rightarrow \mathbb{R}$ localmente Lebesgue-Stieltjes integrável com respeito a $u$ tal que para quaisquer $w, v \in\left[t_{0}, \infty\right)$, temos

$$
\left|\int_{w}^{v} p(s) d u(s)\right| \leq \int_{w}^{v} K(s) d u(s) .
$$

Lembremos ao leitor da definição de solução do problema (3.2) com condição inicial $y_{t_{0}}=\phi$.

Definição 3.6. Considere o problema (3.2) e suponha que valham as condições $\left(H_{1}\right)$ e $\left(H_{4}\right)$. Se uma função $y \in G^{-}\left(\left[t_{0}-r,+\infty\right), \mathbb{R}^{n}\right)$ for tal que

- $y(t)=y\left(t_{0}\right)+\int_{t_{0}}^{t} f\left(y_{s}, s\right) d g(s)+\int_{t_{0}}^{t} p(s) d u(s)$, para quase todo $t \in\left[t_{0},+\infty\right)$;

- $y_{t_{0}}=\phi$,

então y será dita uma solução de $(3.2)$ em $\left[t_{0}-r,+\infty\right)$ com condição inicial $y_{t_{0}}=\phi$.

Os seguintes conceitos de estabilidade da solução trivial da equação (3.1) generalizam o conceito de A. Halanay de estabilidade integral. Veja [16]. Começamos introduzindo um conceito de estabilidade da solução trivial de (3.1) que será essencial para nossos propósitos.

Definição 3.7. A solução $y \equiv 0$ de (3.1) será integralmente estável, se para todo $\varepsilon>0$ existir um $\delta=\delta(\varepsilon)>0$ tal que, se $\phi \in S$ e $p:[\gamma, v] \rightarrow \mathbb{R}^{n}$ forem tais que $\|\phi\|_{\infty}<\delta$ e

$$
\sup _{t \in[\gamma, v]}\left|\int_{\gamma}^{t} p(s) d u(s)\right|<\delta
$$

então

$$
|\bar{y}(t ; \gamma, \phi)|<\varepsilon, \text { para todo } t \in[\gamma, v],
$$

onde $\bar{y}(t ; \gamma, \phi)$ é uma solução da equação perturbada (3.2) tal que $\bar{y}_{\gamma}=\phi$.

Definição 3.8. A solução $y \equiv 0$ de (3.1) será integralmente atratora, se existir um $\widetilde{\delta}>0$ e para todo $\varepsilon>0$, existirem um $T=T(\varepsilon) \geq 0$ e um $\rho=\rho(\varepsilon)>0$ tais que, se $\phi \in S$ e $p:[\gamma, v] \rightarrow \mathbb{R}^{n}$ forem tais que

$$
\|\phi\|_{\infty}<\widetilde{\delta} \quad e \quad \sup _{t \in[\gamma, v]}\left|\int_{\gamma}^{t} p(s) d u(s)\right|<\rho
$$

então

$$
|\bar{y}(t ; \gamma, \phi)|<\varepsilon, \text { para todo } t \geq \gamma+T, t \in[\gamma, v],
$$


onde $\bar{y}(t ; \gamma, \phi)$ é uma solução da equação $(3.2)$ com $\bar{y}_{\gamma}=\phi$.

Definição 3.9. A solução $y \equiv 0$ de (3.1) será dita integralmente assintoticamente estável, se ela for integralmente estável e integralmente atratora.

Note que, se a solução nula $y \equiv 0$ da equação não perturbada (3.1) for integralmente estável, então $y \equiv 0$ será uniformemente estável no sentido usual de Lyapunov.

\subsection{EDFs em medida vistas como EDOGs}

Nesta seção, estabelecemos uma relação entre EDFs em medida e EDOGs. Tal relação pode ser encontrada em [11].

Lembremos que $S=\left\{y_{t} ; y \in O, t \in\left[t_{0}, \infty\right)\right\}$, onde $O \subset G\left(\left[t_{0}-r,+\infty\right), \mathbb{R}^{n}\right)$ tem a propriedade do prolongamento.

Suponha que $f: S \times\left[t_{0}, \infty\right) \rightarrow \mathbb{R}^{n}$ satisfaça as condições $\left(H_{1}\right),\left(H_{2}\right)$ e $\left(H_{3}\right)$ e $p:\left[t_{0}, \infty\right) \rightarrow \mathbb{R}^{n}$ satisfaça as condições $\left(H_{4}\right)$ e $\left(H_{5}\right)$. Mais ainda, suponha que as funções $g, u:\left[t_{0}, \infty\right) \rightarrow \mathbb{R}$ sejam não decrescentes.

Para $y \in O$ e $t \in\left[t_{0},+\infty\right)$, defina

$$
F(y, t)(\vartheta)=\left\{\begin{array}{l}
0, \quad t_{0}-r \leq \vartheta \leq t_{0} \\
\int_{t_{0}}^{\vartheta} f\left(y_{s}, s\right) d g(s), \quad t_{0} \leq \vartheta \leq t<+\infty \\
\int_{t_{0}}^{t} f\left(y_{s}, s\right) d g(s), \quad t \leq \vartheta<+\infty
\end{array}\right.
$$

e

$$
P(t)(\vartheta)=\left\{\begin{array}{l}
0, \quad t_{0}-r \leq \vartheta \leq t_{0} \\
\int_{t_{0}}^{\vartheta} p(s) d u(s), \quad t_{0} \leq \vartheta \leq t<+\infty \\
\int_{t_{0}}^{t} p(s) d u(s), \quad t \leq \vartheta<+\infty
\end{array}\right.
$$

Então,

$$
G(y, t)=F(y, t)+P(t)
$$

define um elemento $G(y, t)$ de $G\left(\left[t_{0}-r,+\infty\right), \mathbb{R}^{n}\right)$ e $G(y, t)(\vartheta) \in \mathbb{R}^{n}$ é o valor de $G(y, t)$ no ponto $\vartheta \in\left[t_{0}-r,+\infty\right)$, ou seja,

$$
G: O \times\left[t_{0},+\infty\right) \rightarrow G\left(\left[t_{0}-r,+\infty\right), \mathbb{R}^{n}\right)
$$


Considere a seguinte EDOG

$$
\frac{d x}{d \tau}=D G(x, t)
$$

onde a função $G$ é dada por (3.6).

Seja $h:\left[t_{0},+\infty\right) \rightarrow \mathbb{R}$ definida por

$$
h(t)=\int_{t_{0}}^{t}[M(s)+L(s)] d g(s)+\int_{t_{0}}^{t} K(s) d u(s), \quad t \in\left[t_{0},+\infty\right) .
$$

Então a função $h$ é não decrescente.

Nas condições acima, é fácil mostrar que $G$, dada por (3.6), pertence à classe $\mathcal{F}(\Omega, h)$, onde $\Omega=O \times\left[t_{0},+\infty\right)$. Veja [11] para os detalhes da demonstração deste fato.

Nos resultados a seguir, consideraremos que as funções envolvidas estão definidas no intervalo $\left[t_{0}, t_{0}+\sigma\right]$, com $\sigma>0$, e não mais em $\left[t_{0},+\infty\right)$ para facilitar as demonstrações.

Uma demonstração do próximo resultado pode ser encontrada em [11], Lema 4.1.

Lema 3.10. Seja $O \subset G\left(\left[t_{0}-r, t_{0}+\sigma\right], \mathbb{R}^{n}\right)$ com a propriedade do prolongamento $e$ $S=\left\{y_{t} ; y \in O, t \in\left[t_{0}, t_{0}+\sigma\right]\right\}$. Suponha que $\phi \in S$ e que $g, u:\left[t_{0}, t_{0}+\sigma\right] \rightarrow \mathbb{R}$ sejam funções não decrescentes. Sejam $f: S \times\left[t_{0}, t_{0}+\sigma\right] \rightarrow \mathbb{R}^{n}$ tal que a integral $\int_{t_{0}}^{t_{0}+\sigma} f\left(y_{t}, t\right) d g(t)$ exista para todo $y \in O$ e $p:\left[t_{0}, t_{0}+\sigma\right] \rightarrow \mathbb{R}^{n}$ tal que a integral $\int_{t_{0}}^{t_{0}+\sigma} p(t) d u(t)$ exista. Considere $G$ dada por (3.6) e suponha que $x:\left[t_{0}, t_{0}+\sigma\right] \rightarrow O$ seja uma solução de

$$
\frac{d x}{d \tau}=D G(x, t)
$$

com condição inicial

$$
\left\{\begin{array}{l}
x\left(t_{0}\right)(\vartheta)=\phi\left(\vartheta-t_{0}\right), \quad \vartheta \in\left[t_{0}-r, t_{0}\right] \\
x\left(t_{0}\right)(\vartheta)=x\left(t_{0}\right)\left(t_{0}\right), \quad \vartheta \in\left[t_{0}, t_{0}+\sigma\right] .
\end{array}\right.
$$

Se $\nu \in\left[t_{0}, t_{0}+\sigma\right]$ e $\vartheta \in\left[t_{0}-r, t_{0}+\sigma\right]$, então

$$
x(\nu)(\vartheta)=x(\nu)(\nu), \quad \vartheta \geq \nu,
$$

e

$$
x(\nu)(\vartheta)=x(\vartheta)(\vartheta), \quad \nu \geq \vartheta
$$


O resultado a seguir apresenta uma correspondência entre EDOGs e EDFs em medida. Tal resultado é uma generalização dos Teoremas 3.8 e 3.9 de [10] e pode ser encontrado em [11], Teorema 4.2.

Teorema 3.11. Sejam $O \subset G\left(\left[t_{0}-r, t_{0}+\sigma\right], \mathbb{R}^{n}\right)$ com a propriedade do prolongamento, $S=\left\{x_{t} ; x \in O, t \in\left[t_{0}, t_{0}+\sigma\right]\right\}$ e $\phi \in S$. Suponha que $g:\left[t_{0}, t_{0}+\sigma\right] \rightarrow \mathbb{R} e$ $u:\left[t_{0}, t_{0}+\sigma\right] \rightarrow \mathbb{R}$ sejam funções não decrescentes, $f: S \times\left[t_{0}, t_{0}+\sigma\right] \rightarrow \mathbb{R}^{n}$ satisfaça as condições $\left(H_{1}\right),\left(H_{2}\right),\left(H_{3}\right)$ e $p:\left[t_{0}, t_{0}+\sigma\right] \rightarrow \mathbb{R}^{n}$ satisfaça as condições $\left(H_{4}\right)$ e $\left(H_{5}\right)$.

(i) Seja y: $\left[t_{0}-r, t_{0}+\sigma\right] \rightarrow \mathbb{R}^{n}$ uma solução da equação diferencial funcional em medida com perturbações

$$
\left\{\begin{array}{l}
D y=f\left(y_{t}, t\right) D g+p(t) D u, \quad t \in\left[t_{0}, t_{0}+\sigma\right] \\
y_{t_{0}}=\phi
\end{array}\right.
$$

Para todo $t \in\left[t_{0}-r, t_{0}+\sigma\right]$, seja

$$
x(t)(\vartheta)= \begin{cases}y(\vartheta), & \vartheta \in\left[t_{0}-r, t\right], \\ y(t), & \vartheta \in\left[t, t_{0}+\sigma\right] .\end{cases}
$$

Então a função $x:\left[t_{0}, t_{0}+\sigma\right] \rightarrow G\left(\left[t_{0}-r, t_{0}+\sigma\right], \mathbb{R}^{n}\right)$ será uma solução da EDOG

$$
\frac{d x}{d \tau}=D G(x, t)
$$

com G dada por (3.6), com

$$
x\left(t_{0}\right)(\vartheta)=\left\{\begin{array}{l}
\phi\left(\vartheta-t_{0}\right), t_{0}-r \leq \vartheta \leq t_{0}, \\
\phi(0), t_{0} \leq \vartheta<t_{0}+\sigma .
\end{array}\right.
$$

(ii) Reciprocamente, seja $G$ dada por (3.6). Suponha que $x:\left[t_{0}, t_{0}+\sigma\right] \rightarrow O$ seja uma solução da EDOG

$$
\frac{d x}{d \tau}=D G(x, t)
$$

com a seguinte condição inicial

$$
x\left(t_{0}\right)(\vartheta)=\left\{\begin{array}{l}
\phi\left(\vartheta-t_{0}\right), t_{0}-r \leq \vartheta \leq t_{0}, \\
\phi(0), t_{0} \leq \vartheta<t_{0}+\sigma .
\end{array}\right.
$$


Então a função $y:\left[t_{0}, t_{0}+\sigma\right] \rightarrow \mathbb{R}^{n}$ definida por

$$
y(\vartheta)=\left\{\begin{array}{l}
x\left(t_{0}\right)(\vartheta), t_{0}-r \leq \vartheta \leq t_{0} \\
x(\vartheta)(\vartheta), \quad t_{0} \leq \vartheta<t_{0}+\sigma
\end{array}\right.
$$

será uma solução da equação diferencial funcional em medida com perturbações

$$
\left\{\begin{array}{l}
D y=f\left(y_{t}, t\right) D g+p(t) D u, \quad t \in\left[t_{0}, t_{0}+\sigma\right] \\
y_{t_{0}}=\phi
\end{array}\right.
$$

Demonstração: $\quad$ Para mostrarmos (i), basta provarmos que, para qualquer $v \in$ $\left[t_{0}, t_{0}+\sigma\right]$, a integral $\int_{t_{0}}^{v} D G(x(\tau), t)$ existe e vale

$$
x(v)-x\left(t_{0}\right)=\int_{t_{0}}^{v} D G(x(\tau), t)
$$

Seja $\varepsilon>0$ dado. Defina a função $h:\left[t_{0}, t_{0}+\sigma\right] \rightarrow \mathbb{R}$ por

$$
h(t)=\int_{t_{0}}^{t}[L(s)+M(s)] d g(s)+\int_{t_{0}}^{t} K(s) d u(s)
$$

onde $M, L$ e $K$ foram dadas nas condições $\left(H_{2}\right),\left(H_{3}\right)$ e $\left(H_{5}\right)$ respectivamente. Como as funções $g$ e $u$ são não decrescentes, elas podem ter apenas um número finito de pontos $t \in\left[t_{0}, v\right]$ tais que $\Delta^{+} g(t) \geq \varepsilon$ e $\Delta^{+} u(t) \geq \varepsilon$. O mesmo vale para a função $h$, ou seja, $h$ pode ter apenas um número finito de pontos $t \in\left[t_{0}, v\right]$ tais que $\Delta^{+} h(t) \geq \varepsilon$. Note que os pontos que satisfazem esta última desigualdade são a união dos pontos que satisfazem a desigualdade para as funções $g$ e $u$, pela definição da função $h$. Denotemos estes pontos por $t_{1}, \ldots, t_{m}$.

Considere um calibre $\delta:\left[t_{0}, t_{0}+\sigma\right] \rightarrow \mathbb{R}^{+}$tal que

$$
\delta(\tau)<\min \left\{\frac{t_{k}-t_{k-1}}{2}, k=2, \ldots, m\right\}, \quad \tau \in\left[t_{0}, t_{0}+\sigma\right]
$$

e

$$
\delta(\tau)<\min \left\{\left|\tau-t_{k}\right|,\left|\tau-t_{k-1}\right| ; \tau \in\left(t_{k-1}, t_{k}\right), \quad k=2, \ldots, m\right\}, \quad \tau \in\left[t_{0}, t_{0}+\sigma\right]
$$

Estas condições asseguram que, se um par ponto-intervalo $(\tau,[c, d])$ for $\delta$-fino, então o intervalo $[c, d]$ conterá no máximo um dos pontos $t_{1}, \ldots, t_{m}$, e, mais ainda, teremos $\tau=t_{k}$ sempre que $t_{k} \in[c, d]$. 
Como $y_{t_{k}}=x\left(t_{k}\right)_{t_{k}}$, segue do Teorema 3.2 o fato que

$$
\lim _{s \rightarrow t_{k}+} \int_{t_{k}}^{s} L(s)\left\|y_{s}-x\left(t_{k}\right)_{s}\right\|_{\infty} d g(s)=L\left(t_{k}\right)\left\|y_{t_{k}}-x\left(t_{k}\right)_{t_{k}}\right\|_{\infty} \Delta^{+} g\left(t_{k}\right)=0
$$

para qualquer $k \in\{1, \ldots, m\}$. Assim, o calibre $\delta$ pode ser escolhido de modo que

$$
\int_{t_{k}}^{t_{k}+\delta\left(t_{k}\right)} L(s)\left\|y_{s}-x\left(t_{k}\right)_{s}\right\|_{\infty} d g(s)<\frac{\varepsilon}{2 m+1}, \quad k \in\{1, \ldots, m\} .
$$

Utilizando novamente o Teorema 3.2, obtemos

$$
\begin{gathered}
|y(\tau+t)-y(\tau)|=\left|\int_{\tau}^{\tau+t} f\left(y_{s}, s\right) d g(s)+\int_{\tau}^{\tau+t} p(s) d u(s)\right| \leq \\
\leq \int_{\tau}^{\tau+t} M(s) d g(s)+\int_{\tau}^{\tau+t} K(s) d u(s) \leq h(\tau+t)-h(\tau),
\end{gathered}
$$

e, portanto,

$$
|y(\tau+)-y(\tau)| \leq \Delta^{+} h(\tau)<\varepsilon, \quad \tau \in\left[t_{0}, t_{0}+\sigma\right] \backslash\left\{t_{1}, \ldots, t_{m}\right\}
$$

Assim, podemos assumir que o calibre $\delta$ é tal que

$$
|y(\rho)-y(\tau)| \leq \varepsilon
$$

para quaisquer $\tau \in\left[t_{0}, t_{0}+\sigma\right] \backslash\left\{t_{1}, \ldots, t_{m}\right\}$ e $\rho \in[\tau, \tau+\delta(\tau))$.

Suponha, agora, que $\left\{\left(\tau_{i},\left[s_{i-1}, s_{i}\right]\right), i=1, \ldots, l\right\}$ seja uma partição marcada $\delta$-fina do intervalo $\left[t_{0}, v\right]$. Utilizando a definição de $x$, podemos mostrar facilmente que

$$
\left[x\left(s_{i}\right)-x\left(s_{i-1}\right)\right](\vartheta)= \begin{cases}0, & \vartheta \in\left[t_{0}-r, s_{i-1}\right], \\ \int_{s_{i-1}}^{\vartheta} f\left(y_{s}, s\right) d g(s)+\int_{s_{i-1}}^{\vartheta} p(s) d u(s), & \vartheta \in\left[s_{i-1}, s_{i},\right] \\ \int_{s_{i-1}}^{s_{i}} f\left(y_{s}, s\right) d g(s)+\int_{s_{i-1}}^{s_{i}} p(s) d u(s), & \vartheta \in\left[s_{i}, t_{0}+\sigma\right] .\end{cases}
$$

De forma análoga, segue da definição de $G$ que

$$
\left[G\left(x\left(\tau_{i}\right), s_{i}\right)-G\left(x\left(\tau_{i}\right), s_{i-1}\right)\right](\vartheta)=
$$




$$
= \begin{cases}0, & \vartheta \in\left[t_{0}-r, s_{i-1}\right], \\ \int_{s_{i-1}}^{\vartheta} f\left(x\left(\tau_{i}\right)_{s}, s\right) d g(s)+\int_{s_{i-1}}^{\vartheta} p(s) d u(s), & \vartheta \in\left[s_{i-1}, s_{i}\right], \\ \int_{s_{i-1}}^{s_{i}} f\left(x\left(\tau_{i}\right)_{s}, s\right) d g(s)+\int_{s_{i-1}}^{s_{i}} p(s) d u(s), & \vartheta \in\left[s_{i}, t_{0}+\sigma\right] .\end{cases}
$$

Daí, combinando as igualdades anteriores, obtemos

$$
\begin{aligned}
& {\left[x\left(s_{i}\right)-x\left(s_{i-1}\right)\right](\vartheta)-\left[G\left(x\left(\tau_{i}\right), s_{i}\right)-G\left(x\left(\tau_{i}\right), s_{i-1}\right)\right](\vartheta)=} \\
& = \begin{cases}0, & \vartheta \in\left[t_{0}-r, s_{i-1}\right], \\
\int_{s_{i-1}}^{\vartheta}\left[f\left(y_{s}, s\right)-f\left(x\left(\tau_{i}\right)_{s}, s\right)\right] d g(s), & \vartheta \in\left[s_{i-1}, s_{i}\right], \\
\int_{s_{i-1}}^{s_{i}}\left[f\left(y_{s}, s\right)-f\left(x\left(\tau_{i}\right)_{s}, s\right)\right] d g(s), & \vartheta \in\left[s_{i}, t_{0}+\sigma\right] .\end{cases}
\end{aligned}
$$

Assim,

$$
\begin{gathered}
\left\|x\left(s_{i}\right)-x\left(s_{i-1}\right)-\left[G\left(x\left(\tau_{i}\right), s_{i}\right)-G\left(x\left(\tau_{i}\right), s_{i-1}\right)\right]\right\|_{\infty}= \\
=\sup _{\vartheta \in\left[t_{0}-r, t_{0}+\sigma\right]}\left|\left[x\left(s_{i}\right)-x\left(s_{i-1}\right)\right](\vartheta)-\left[G\left(x\left(\tau_{i}\right), s_{i}\right)-G\left(x\left(\tau_{i}\right), s_{i-1}\right)\right](\vartheta)\right|= \\
=\sup _{\vartheta \in\left[s_{i-1}, s_{i}\right]}\left|\int_{s_{i-1}}^{\vartheta}\left[f\left(y_{s}, s\right)-f\left(x\left(\tau_{i}\right)_{s}, s\right)\right] d g(s)\right| .
\end{gathered}
$$

Pela definição de $x, x\left(\tau_{i}\right)_{s}=y_{s}$ sempre que $s \leq \tau_{i}$. Logo

$$
\int_{s_{i-1}}^{\vartheta}\left[f\left(y_{s}, s\right)-f\left(x\left(\tau_{i}\right)_{s}, s\right)\right] d g(s)= \begin{cases}0, & \vartheta \in\left[s_{i-1}, \tau_{i}\right] \\ \int_{\tau_{i}}^{\vartheta}\left[f\left(y_{s}, s\right)-f\left(x\left(\tau_{i}\right)_{s}, s\right)\right] d g(s), & \vartheta \in\left[\tau_{i}, s_{i}\right] .\end{cases}
$$

Então a condição $\left(H_{3}\right)$ implica

$$
\begin{aligned}
\left|\int_{\tau_{i}}^{\vartheta}\left[f\left(y_{s}, s\right)-f\left(x\left(\tau_{i}\right)_{s}, s\right)\right] d g(s)\right| & \leq \int_{\tau_{i}}^{\vartheta} L(s)\left\|y_{s}-x\left(\tau_{i}\right)_{s}\right\|_{\infty} d g(s) \\
& \leq \int_{\tau_{i}}^{s_{i}} L(s)\left\|y_{s}-x\left(\tau_{i}\right)_{s}\right\|_{\infty} d g(s)
\end{aligned}
$$

Dado um par ponto-intervalo particular $\left(\tau_{i},\left[s_{i-1}, s_{i}\right]\right)$, existem duas possibilidades:

(a) A interseção de $\left[s_{i-1}, s_{i}\right]$ e $\left\{t_{1}, \ldots, t_{m}\right\}$ contém um único ponto $t_{k}=\tau_{i}$.

(b) A interseção de $\left[s_{i-1}, s_{i}\right]$ e $\left\{t_{1}, \ldots, t_{m}\right\}$ é vazia. 
Caso (a) ocorra, segue da definição do calibre $\delta$ que

$$
\int_{\tau_{i}}^{s_{i}} L(s)\left\|y_{s}-x\left(\tau_{i}\right)_{s}\right\|_{\infty} d g(s) \leq \frac{\varepsilon}{2 m+1}
$$

ou seja,

$$
\left\|x\left(s_{i}\right)-x\left(s_{i-1}\right)-\left[G\left(x\left(\tau_{i}\right), s_{i}\right)-G\left(x\left(\tau_{i}\right), s_{i-1}\right)\right]\right\|_{\infty} \leq \frac{\varepsilon}{2 m+1} .
$$

Caso (b) ocorra, temos

$$
\left\|y_{s}-x\left(\tau_{i}\right)_{s}\right\|_{\infty}=\sup _{\rho \in\left[\tau_{i}, s\right]}\left|y(\rho)-y\left(\tau_{i}\right)\right| \leq \varepsilon, \quad s \in\left[\tau_{i}, s_{i}\right]
$$

pela definição do calibre $\delta$. Assim

$$
\left\|x\left(s_{i}\right)-x\left(s_{i-1}\right)-\left[G\left(x\left(\tau_{i}\right), s_{i}\right)-G\left(x\left(\tau_{i}\right), s_{i-1}\right)\right]\right\|_{\infty} \leq \varepsilon \int_{\tau_{i}}^{s_{i}} L(s) d g(s) .
$$

Combinando os casos (a) e (b) e utilizando o fato de que (a) ocorre no máximo $2 m$ vezes, obtemos

$$
\left\|x(v)-x\left(t_{0}\right)-\sum_{i=1}^{l}\left[G\left(x\left(\tau_{i}\right), s_{i}\right)-G\left(x\left(\tau_{i}\right), s_{i-1}\right)\right]\right\|_{\infty} \leq \varepsilon \int_{t_{0}}^{t_{0}+\sigma} L(s) d g(s)+\frac{2 m \varepsilon}{2 m+1} .
$$

Como $\varepsilon$ é arbitrário, temos

$$
x(v)-x\left(t_{0}\right)=\int_{t_{0}}^{v} D G(x(\tau), t), \quad \vartheta \in\left[t_{0}, t_{0}+\sigma\right]
$$

como queríamos.

Agora vamos mostrar (ii). A igualdade $y_{t_{0}}=\phi$ segue facilmente das definições de $y$ e $x\left(t_{0}\right)$. Resta mostrarmos que vale

$$
y(v)-y\left(t_{0}\right)=\int_{t_{0}}^{v} f\left(y_{s}, s\right) d g(s)+\int_{t_{0}}^{v} p(s) d u(s), \quad v \in\left[t_{0}, t_{0}+\sigma\right] .
$$

Seja $v \in\left[t_{0}, t_{0}+\sigma\right]$. Utilizando o Lema 3.10, obtemos

$$
y(v)-y\left(t_{0}\right)=x(v)(v)-x\left(t_{0}\right)\left(t_{0}\right)=x(v)(v)-x\left(t_{0}\right)(v)=\left(\int_{t_{0}}^{v} D G(x(\tau), t)\right)(v) .
$$


Assim

$$
\begin{gathered}
y(v)-y\left(t_{0}\right)-\int_{t_{0}}^{v} f\left(y_{s}, s\right) d g(s)+\int_{t_{0}}^{v} p(s) d u(s)= \\
=\left(\int_{t_{0}}^{v} D G(x(\tau), t)\right)(v)-\int_{t_{0}}^{v} f\left(y_{s}, s\right) d g(s)-\int_{t_{0}}^{v} p(s) d u(s) .
\end{gathered}
$$

Defina a função $h:\left[t_{0}, t_{0}+\sigma\right] \rightarrow \mathbb{R}$ por

$$
h(t)=\int_{t_{0}}^{t}(L(s)+M(s)) d g(s)+\int_{t_{0}}^{t} K(s) d u(s) .
$$

Seja $\varepsilon>0$ arbitrário. Como $g$ e $u$ são funções não decrescentes, elas podem ter no máximo um número finito de pontos $t \in\left[t_{0}, v\right]$ tais que $\Delta^{+} g(t) \geq \varepsilon$ e $\Delta^{+} u(t) \geq \varepsilon$. Analogamente, temos o mesmo para a função $h$. Denotamos a união dos pontos de descontinuidade das funções $g$ e $u$ por $t_{1}, \ldots, t_{m}$. (Lembrando que estes pontos são os mesmos para a função $h$ ).

Considere um calibre $\delta:\left[t_{0}, t_{0}+\sigma\right] \rightarrow(0,+\infty)$ tal que

$$
\delta(\tau)<\min \left\{\frac{t_{k}-t_{k-1}}{2}, k=2, \ldots, m\right\}, \quad \tau \in\left[t_{0}, t_{0}+\sigma\right]
$$

$\mathrm{e}$

$$
\delta(\tau)<\min \left\{\left|\tau-t_{k}\right| ; k=1, \ldots, m\right\}, \quad \tau \in\left[t_{0}, t_{0}+\sigma\right] .
$$

Estas condições asseguram que, se um par ponto-intervalo $(\tau,[c, d])$ for $\delta$-fino, então $[c, d]$ conterá no máximo um dos pontos $t_{1}, \ldots, t_{m}$, e, ainda mais, $\tau=t_{k}$ sempre que $t_{k} \in[c, d]$.

Novamente, o calibre $\delta$ pode ser escolhido de forma que

$$
\int_{t_{k}}^{t_{k}+\delta\left(t_{k}\right)} L(s)\left\|y_{s}-x\left(t_{k}\right)_{s}\right\|_{\infty} d g(s)<\frac{\varepsilon}{2 m+1}, \quad k \in\{1, \ldots, m\} .
$$

Como descrito anteriormente, a função $G$ dada por (3.4) pertence à classe $\mathcal{F}(O \times$ $\left.\left[t_{0}, t_{0}+\sigma\right], h\right)$. Como

$$
|h(\tau+)-h(\tau)| \leq \varepsilon, \quad \tau \in\left[t_{0}, t_{0}+\sigma\right] \backslash\left\{t_{1}, \ldots, t_{m}\right\}
$$

podemos assumir que o calibre $\delta$ satisfaz

$$
|h(\rho)-h(\tau)| \leq \varepsilon \text { para qualquer } \rho \in[\tau, \tau+\delta(\tau)) \text {. }
$$


Finalmente, o calibre $\delta$ deve ser tal que

$$
\left\|\int_{t_{0}}^{v} D G(x(\tau), t)-\sum_{i=1}^{l}\left[G\left(x\left(\tau_{i}\right), s_{i}\right)-G\left(x\left(\tau_{i}\right), s_{i-1}\right)\right]\right\|_{\infty}<\varepsilon
$$

para toda partição $\delta$-fina $\left\{\left(\tau_{i},\left[s_{i-1}, s_{i}\right]\right), i=1, \ldots, l\right\}$ de $\left[t_{0}, v\right]$. A existência de tal calibre segue da definição da integral de Kurzweil. Escolhemos uma partição $\delta$-fina particular $\left\{\left(\tau_{i},\left[s_{i-1}, s_{i}\right]\right), i=1, \ldots, l\right\}$ de $\left[t_{0}, v\right]$. Daí, por (3.13) e (3.14), temos

$$
\begin{gathered}
\left|y(v)-y\left(t_{0}\right)-\int_{t_{0}}^{v} f\left(y_{s}, s\right) d g(s)-\int_{t_{0}}^{v} p(s) d u(s)\right|= \\
=\left|\left(\int_{t_{0}}^{v} D G(x(\tau), t)\right)(v)-\int_{t_{0}}^{v} f\left(y_{s}, s\right) d g(s)-\int_{t_{0}}^{v} p(s) d u(s)\right|< \\
<\varepsilon+\left|\sum_{i=1}^{l}\left[G\left(x\left(\tau_{i}\right), s_{i}\right)-G\left(x\left(\tau_{i}\right), s_{i-1}\right)\right](v)-\int_{t_{0}}^{v} f\left(y_{s}, s\right) d g(s)-\int_{t_{0}}^{v} p(s) d u(s)\right| \leq \\
\leq \varepsilon+\sum_{i=1}^{l}\left|\left[G\left(x\left(\tau_{i}\right), s_{i}\right)-G\left(x\left(\tau_{i}\right), s_{i-1}\right)\right](v)-\int_{s_{i-1}}^{s_{i}} f\left(y_{s}, s\right) d g(s)-\int_{s_{i-1}}^{s_{i}} p(s) d u(s)\right| .
\end{gathered}
$$

A definição de $G$ nos leva a

$$
\left[G\left(x\left(\tau_{i}\right), s_{i}\right)-G\left(x\left(\tau_{i}\right), s_{i-1}\right)\right](v)=\int_{s_{i-1}}^{s_{i}} f\left(x\left(\tau_{i}\right)_{s}, s\right) d g(s)+\int_{s_{i-1}}^{s_{i}} p(s) d u(s)
$$

o que implica

$$
\begin{gathered}
\left|\left[G\left(x\left(\tau_{i}\right), s_{i}\right)-G\left(x\left(\tau_{i}\right), s_{i-1}\right)\right](v)-\int_{s_{i-1}}^{s_{i}} f\left(y_{s}, s\right) d g(s)-\int_{s_{i-1}}^{s_{i}} p(s) d u(s)\right|= \\
=\left|\int_{s_{i-1}}^{s_{i}}\left[f\left(x\left(\tau_{i}\right)_{s}, s\right)-f\left(y_{s}, s\right)\right] d g(s)\right| .
\end{gathered}
$$

Pelo Lema 3.10, para qualquer $i \in\{1, \ldots, l\}$, temos $x\left(\tau_{i}\right)_{s}=x(s)_{s}=y_{s}$ para $s \in\left[s_{i-1}, \tau_{i}\right]$ e $y_{s}=x(s)_{s}=x\left(s_{i}\right)_{s}$ para $s \in\left[\tau_{i}, s_{i}\right]$. Portanto

$$
\begin{aligned}
& \left|\int_{s_{i-1}}^{s_{i}}\left[f\left(x\left(\tau_{i}\right)_{s}, s\right)-f\left(y_{s}, s\right)\right] d g(s)\right|=\left|\int_{\tau_{i}}^{s_{i}}\left[f\left(x\left(\tau_{i}\right)_{s}, s\right)-f\left(y_{s}, s\right)\right] d g(s)\right|= \\
= & \left|\int_{\tau_{i}}^{s_{i}}\left[f\left(x\left(\tau_{i}\right)_{s}, s\right)-f\left(x\left(s_{i}\right)_{s}, s\right)\right] d g(s)\right| \leq \int_{\tau_{i}}^{s_{i}} L(s)\left\|x\left(\tau_{i}\right)_{s}-x\left(s_{i}\right)_{s}\right\|_{\infty} d g(s),
\end{aligned}
$$

onde a última desigualdade segue da condição $\left(H_{3}\right)$. 
Novamente, separamos em dois casos:

(a) A interseção de $\left[s_{i-1}, s_{i}\right]$ e $\left\{t_{1}, \ldots, t_{m}\right\}$ contém um único ponto $t_{k}=\tau_{i}$.

(b) A interseção de $\left[s_{i-1}, s_{i}\right]$ e $\left\{t_{1}, \ldots, t_{m}\right\}$ é vazia.

Caso (a) ocorra, segue da definiçaõ do calibre $\delta$ que

$$
\int_{\tau_{i}}^{s_{i}} L(s)\left\|y_{s}-x\left(\tau_{i}\right)_{s}\right\|_{\infty} d g(s) \leq \frac{\varepsilon}{2 m+1},
$$

ou seja,

$$
\left|\left[G\left(x\left(\tau_{i}\right), s_{i}\right)-G\left(x\left(\tau_{i}\right), s_{i-1}\right)\right](v)-\int_{s_{i-1}}^{s_{i}} f\left(y_{s}, s\right) d g(s)-\int_{s_{i-1}}^{s_{i}} p(s) d u(s)\right| \leq \frac{\varepsilon}{2 m+1} .
$$

Caso (b) ocorra, como toda solução da EDOG é regrada, obtemos a seguinte estimativa

$$
\left\|x\left(s_{i}\right)_{s}-x\left(\tau_{i}\right)_{s}\right\|_{\infty} \leq\left\|x\left(s_{i}\right)-x\left(\tau_{i}\right)\right\|_{\infty} \leq h\left(s_{i}\right)-h\left(\tau_{i}\right) \leq \varepsilon
$$

para qualquer $s \in\left[\tau_{i}, s_{i}\right]$, e assim

$$
\begin{gathered}
\left|\left[G\left(x\left(\tau_{i}\right), s_{i}\right)-G\left(x\left(\tau_{i}\right), s_{i-1}\right)\right](v)-\int_{s_{i-1}}^{s_{i}} f\left(y_{s}, s\right) d g(s)-\int_{s_{i-1}}^{s_{i}} p(s) d u(s)\right| \leq \\
\leq \varepsilon \int_{\tau_{i}}^{s_{i}} L(s) d g(s) .
\end{gathered}
$$

Combinando os casos (a) e (b) e utilizando o fato de que o caso (a) ocorre no máximo $2 m$ vezes, obtemos

$$
\begin{gathered}
\sum_{i=1}^{l}\left|\left[G\left(x\left(\tau_{i}\right), s_{i}\right)-G\left(x\left(\tau_{i}\right), s_{i-1}\right)\right](v)-\int_{s_{i-1}}^{s_{i}} f\left(y_{s}, s\right) d g(s)-\int_{s_{i-1}}^{s_{i}} p(s) d u(s)\right| \leq \\
\leq \varepsilon \int_{t_{0}}^{t_{0}+\sigma} L(s) d g(s)+\frac{2 m \varepsilon}{2 m+1}<\varepsilon\left(1+\int_{t_{0}}^{t_{0}+\sigma} L(s) d g(s)\right) .
\end{gathered}
$$

Consequentemente,

$$
\left|y(v)-y\left(t_{0}\right)-\int_{t_{0}}^{v} f\left(y_{s}, s\right) d g(s)-\int_{s_{i-1}}^{s_{i}} p(s) d u(s)\right|<\varepsilon\left(2+\int_{t_{0}}^{t_{0}+\sigma} L(s) d g(s)\right),
$$

o que completa a demonstração. 
Agora usaremos o Teorema 1.6 e o Teorema 3.11 para obter um teorema de existência local e unicidade de soluções para EDFs em medida. Tal resultado pode ser encontrado em [10], Teorema 5.3.

Teorema 3.12. Suponha que $O \subset G\left(\left[t_{0}-r, t_{0}+\sigma\right], \mathbb{R}^{n}\right)$ tenha a propriedade do prolongamento, $S=\left\{x_{t} ; x \in O, t \in\left[t_{0}, t_{0}+\sigma\right]\right\}, g:\left[t_{0}, t_{0}+\sigma\right] \rightarrow \mathbb{R}$ seja contínua à esquerda e não decrescente, $f: S \times\left[t_{0}, t_{0}+\sigma\right] \rightarrow \mathbb{R}^{n}$ satisfaça as condições $\left(H_{1}\right),\left(H_{2}\right)$, $\left(H_{3}\right)$. Se $\phi \in S$ for tal que a função

$$
z(t)= \begin{cases}\phi\left(t-t_{0}\right), & t \in\left[t_{0}-r, t_{0}\right] \\ \phi(0)+f\left(\phi, t_{0}\right) \Delta^{+} g\left(t_{0}\right), & t \in\left(t_{0}, t_{0}+\sigma\right]\end{cases}
$$

pertence à $O$, então existirá $\Delta>0$ e uma função $y:\left[t_{0}-r, t_{0}+\Delta\right] \rightarrow \mathbb{R}^{n}$ que é a única solução da equação diferencial funcional em medida

$$
\left\{\begin{array}{l}
D y=f\left(y_{t}, t\right) D g \\
y_{t_{0}}=\phi
\end{array}\right.
$$

Demonstração: Esta demonstração segue as ideias da demonstração do Teorema 5.3 de [10].

Como visto anteriormente, a função $F$ dada por (3.4) pertence à classe $\mathcal{F}(O \times$ $\left.\left[t_{0}, t_{0}+\sigma\right], h\right) \mathrm{com}$

$$
h(t)=\int_{t_{0}}^{t}[M(s)+L(s)] d g(s), \quad t \in\left[t_{0}, t_{0}+\sigma\right] .
$$

Seja

$$
x\left(t_{0}\right)(\vartheta)=\left\{\begin{array}{l}
\phi\left(\vartheta-t_{0}\right), t_{0}-r \leq \vartheta \leq t_{0} \\
\phi(0), t_{0} \leq \vartheta<t_{0}+\sigma .
\end{array}\right.
$$

Então $x\left(t_{0}\right) \in O$. Vamos mostrar que $x\left(t_{0}\right)+F\left(x\left(t_{0}\right), t_{0}+\right)-F\left(x\left(t_{0}\right), t_{0}\right) \in O$. De fato, note que $F\left(x\left(t_{0}\right), t_{0}\right)=0$. O limite $F\left(x\left(t_{0}\right), t_{0}+\right)$ é tomado com respeito a norma do supremo e ele existe, já que $F$ é regrada. Assim é suficiente calcular o limite pontual $F\left(x\left(t_{0}\right), t_{0}+\right)(\vartheta)$ para qualquer $\vartheta \in\left[t_{0}-r, t_{0}+\sigma\right]$.

Pelo Teorema 3.2, obtemos

$$
F\left(x\left(t_{0}\right), t_{0}+\right)(\vartheta)= \begin{cases}0, & t \in\left[t_{0}-r, t_{0}\right], \\ f\left(\phi, t_{0}\right) \Delta^{+} g\left(t_{0}\right), & t \in\left(t_{0}, t_{0}+\sigma\right] .\end{cases}
$$


e, portanto, $x\left(t_{0}\right)+F\left(x\left(t_{0}\right), t_{0}+\right)-F\left(x\left(t_{0}\right), t_{0}\right) \in O$.

Logo, todas as hipóteses do Teorema 1.6 estão satisfeitas e portanto existem $\Delta>0$ e uma única solução $x:\left[t_{0}, t_{0}+\Delta\right] \rightarrow X$ da EDOG

$$
\frac{d x}{d \tau}=D F(x, t), \quad x\left(t_{0}\right)=x_{0}
$$

Pelo Teorema 3.11, a função $y:\left[t_{0}, t_{0}+\Delta\right] \rightarrow \mathbb{R}^{n}$ dada por

$$
y(\vartheta)=\left\{\begin{array}{l}
x\left(t_{0}\right)(\vartheta), t_{0}-r \leq \vartheta \leq t_{0} \\
x(\vartheta)(\vartheta), \quad t_{0} \leq \vartheta<t_{0}+\Delta
\end{array}\right.
$$

é solução da EDF em medida

$$
\left\{\begin{array}{l}
D y=f\left(y_{t}, t\right) D g \\
y_{t_{0}}=\phi
\end{array}\right.
$$

Esta solução é única pois, caso contrário, o Teorema 3.11 implicaria que $x$ não é a única solução da EDOG (3.16), o que seria um absurdo.

\subsection{Relações entre conceitos de estabilidade para EDOGs e EDFs em medida}

Nesta seção, vamos apresentar alguns conceitos novos de estabilidade para EDOGs e também vamos relacionar estes conceitos com os novos conceitos de estabilidade para EDFs em medida apresentados na primeira seção deste capítulo.

Sejam $X$ um espaço de Banach e $B_{c}=\{y \in X ;\|y\|<c\}$ com $c>0$. Defina $\Omega=B_{c} \times\left[t_{0}, \infty\right)$, onde $r>0$ e $c>0$. Suponha que $F: \Omega \rightarrow X$ pertença à classe $\mathcal{F}\left(\Omega, h_{1}\right)$, com $h_{1}(t)=\int_{t_{0}}^{t}[M(s)+L(s)] d g(s)$ para $t \in\left[t_{0}, \infty\right)$, e $F(0, t)-F(0, s)=0$, para $t, s \in\left[t_{0},+\infty\right)$. Então, para todo $[\gamma, v] \subset\left[t_{0},+\infty\right)$, temos

$$
\int_{\gamma}^{v} D F(0, t)=F(0, v)-F(0, \gamma)=0
$$

o que implica que $x \equiv 0$ é uma solução, em $\left[t_{0},+\infty\right)$, da EDOG

$$
\frac{d x}{d \tau}=D F(x, t)
$$


As definições seguintes de estabilidade regular, atratividade regular e estabilidade assintótica regular são novas na teoria de EDOGs.

Definição 3.13. A solução $x \equiv 0$ de (3.17) será dita regularmente estável, se para todo $\varepsilon>0$, existir $\delta=\delta(\varepsilon)>0$ tal que se $\bar{x}:[\gamma, v] \rightarrow B_{c}, t_{0}-r \leq \gamma<v<+\infty$, for uma função regrada que satisfaz

$$
\|\bar{x}(\gamma)\|<\delta \quad e \quad \sup _{s \in[\gamma, v]}\left\|\bar{x}(s)-\bar{x}(\gamma)-\int_{\gamma}^{s} D F(\bar{x}(\tau), t)\right\|_{\infty}<\delta,
$$

então

$$
\|\bar{x}(t)\|<\varepsilon, \quad t \in[\gamma, v] .
$$

Definição 3.14. A solução $x \equiv 0$ de (3.17) será dita regularmente atratora, se existir um $\delta_{0}>0$ e para todo $\varepsilon>0$, existirem um $T=T(\varepsilon) \geq 0$ e um $\rho=\rho(\varepsilon)>0$ tal que, se $\bar{x}:[\gamma, v] \rightarrow B_{c}, t_{0}-r \leq \gamma<v<+\infty$, for uma função satisfazendo

$$
\|\bar{x}(\gamma)\|<\delta_{0} \quad e \quad \sup _{s \in[\gamma, v]}\left\|\bar{x}(s)-\bar{x}(\gamma)-\int_{\gamma}^{s} D F(\bar{x}(\tau), t)\right\|_{\infty}<\rho,
$$

então

$$
\|\bar{x}(t)\|<\varepsilon, \quad \text { para } t \in[\gamma, v] \cap[\gamma+T,+\infty) \text { e } \gamma \geq t_{0} .
$$

Definição 3.15. A solução $x \equiv 0$ de (3.17) será dita regularmente assintoticamente estável, se for regularmente estável e regularmente atratora.

Além da EDOG (3.17), também consideraremos a EDOG perturbada

$$
\frac{d x}{d \tau}=D[F(x, t)+P(t)]
$$

onde $F: O \times\left[t_{0}, \infty\right) \rightarrow G\left(\left[t_{0}-r, \infty\right), \mathbb{R}^{n}\right)$ e $P:\left[t_{0}, \infty\right) \rightarrow G\left(\left[t_{0}-r, \infty\right), \mathbb{R}^{n}\right)$ são dadas por (3.4) e (3.5) respectivamente. Não é difícil mostrar que a função $G(x, t)=$ $F(x, t)+P(t)$ pertence à classe $\mathcal{F}(\Omega, h)$, onde a função $h:\left[t_{0}, \infty\right) \rightarrow \mathbb{R}$ é dada por (3.8). Mais ainda, pelo Teorema 1.6 temos garantida a existência local e unicidade de solução para (3.18).

Antes das próximas definições, cabe observarmos que, pela definição da integral de Kurzweil, para qualquer $[\gamma, v] \subset\left[t_{0}, \infty\right)$ temos

$$
\int_{\gamma}^{v} D P(t)=\int_{\gamma}^{v} d P(t)=P(v)-P(\gamma),
$$

onde a segunda integral é no sentido de Perron (ou Kurzweil-Henstock). 
Considere, agora, as definições seguintes.

Definição 3.16. A solução $x \equiv 0$ de (3.17) será dita estável com respeito a perturbações, se para todo $\varepsilon>0$, existir $\delta=\delta(\varepsilon)>0$ tal que se $\left\|x_{0}\right\|<\delta$ e $P \in G^{-}([\gamma, v], X)$ com $\sup _{s \in[\gamma, v]}\|P(s)-P(\gamma)\|<\delta$, então

$$
\left\|\bar{x}\left(t, \gamma, x_{0}\right)\right\|<\varepsilon, \quad \text { para todo } t \in[\gamma, v]
$$

onde $\bar{x}\left(t, \gamma, x_{0}\right)$ é uma solução da equação diferencial generalizada perturbada (3.18), $\operatorname{com} \bar{x}\left(\gamma, \gamma, x_{0}\right)=x_{0} e[\gamma, v] \subset\left[t_{0},+\infty\right)$.

Definição 3.17. A solução $x \equiv 0$ de (3.17) será dita atratora com respeito a perturbações, se existir um $\widetilde{\delta}>0$ e para todo $\varepsilon>0$, existirem um $T=T(\varepsilon) \geq 0$ e um $\rho=\rho(\varepsilon)>0$ tal que se

$$
\left\|x_{0}\right\|<\widetilde{\delta} \quad e \quad \sup _{s \in[\gamma, v]}\|P(s)-P(\gamma)\|<\rho
$$

com $P \in G^{-}([\gamma, v], X)$, então

$$
\left\|\bar{x}\left(t, \gamma, x_{0}\right)\right\|<\varepsilon \text { para todo } t \geq \gamma+T, t \in[\gamma, v]
$$

onde $\bar{x}\left(t, \gamma, x_{0}\right)$ é uma solução da equação diferencial generalizada perturbada (3.18), $\operatorname{com} \bar{x}\left(\gamma, \gamma, x_{0}\right)=x_{0} e[\gamma, v] \subset\left[t_{0},+\infty\right)$.

Definição 3.18. A solução $x \equiv 0$ de (3.17) será dita assintoticamente estável com respeito a perturbações, se for estável e atratora com respeito a perturbações.

O resultado seguinte mostra algumas equivalências entre as definições que acabamos de introduzir.

Proposição 3.19. As afirmações seguintes são válidas.

(i) A solução $x \equiv 0$ de (3.17) será regularmente estável se, e somente se, for estável com respeito a perturbações.

(ii) A solução $x \equiv 0$ de (3.17) será regularmente atratora se, e somente se, for atratora com respeito a perturbações.

(iii) A solução $x \equiv 0$ de (3.17) será regularmente assintoticamente estável se, e somente se, for assintoticamente estável com respeito a perturbações. 
Demonstração: Mostremos (i). Suponha que a solução $x \equiv 0$ de (3.17) seja regularmente estável. Sejam $\varepsilon>0$ e a quantidade $\delta=\delta(\varepsilon)>0$ dada de acordo com a Definição 3.13. Seja $x(t)=x\left(t, \gamma, x_{0}\right)$ uma solução da EDOG perturbada (3.18) em $[\gamma, v]$. Então, pela definição de solução da equação (3.18), obtemos

$$
x(s)-x(\gamma)=\int_{\gamma}^{s} D F(x(\tau), t)+P(s)-P(\gamma), \quad s \in[\gamma, v]
$$

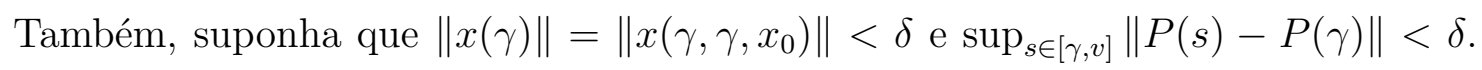
Então (3.19) implica que

$$
x(s)-x(\gamma)-\int_{\gamma}^{s} D F(x(\tau), t)=P(s)-P(\gamma), \quad s \in[\gamma, v]
$$

e portanto,

$$
\sup _{s \in[\gamma, v]}\left\|x(s)-x(\gamma)-\int_{\gamma}^{s} D F(x(\tau), t)\right\|_{\infty}=\sup _{s \in[\gamma, v]}\|P(s)-P(\gamma)\| .
$$

Logo,

$$
\sup _{s \in[\gamma, v]}\left\|x(s)-\int_{\gamma}^{s} D F(x(\tau), t)\right\|_{\infty} \leq\|x(\gamma)\|+\sup _{s \in[\gamma, v]}\|P(s)-P(\gamma)\|<2 \delta .
$$

Assim, a estabilidade regular implica que

$$
\|x(t)\|=\left\|x\left(t, \gamma, x_{0}\right)\right\|<\varepsilon \text { for } t \in[\gamma, v]
$$

e, portanto, a solução trivial de (3.17) é estável com respeito a perturbações.

Reciprocamente, suponha que a solução $x \equiv 0$ de (3.17) seja estável com respeito a perturbações. Seja $\bar{x}:[\gamma, v] \rightarrow B_{c}, t_{0} \leq \gamma<v<+\infty$, contínua à esquerda e regrada em $[\gamma, v]$ tal que $\|\bar{x}(\gamma)\|<\delta$ e

$$
\sup _{s \in[\gamma, v]}\left\|x(s)-\int_{\gamma}^{s} D F(x(\tau), t)\right\|_{\infty}<\delta
$$

onde $\delta>0$ corresponde a algum $\varepsilon>0$ da Definição 3.16. 
Para $s \in[\gamma, v]$, seja $P(s)=P(\gamma)+\bar{x}(s)-\bar{x}(\gamma)-\int_{\gamma}^{s} D F(\bar{x}(\tau), t)$. Então, para $s_{1}, s_{2} \in[\gamma, v]$, temos

$$
\bar{x}\left(s_{2}\right)-\bar{x}\left(s_{1}\right)-\int_{s_{1}}^{s_{2}} D F(\bar{x}(\tau), t)=P\left(s_{2}\right)-P\left(s_{1}\right),
$$

o que implica que $\bar{x}$ é uma solução de (3.18) em $[\gamma, v]$. Mais ainda, temos

$$
\begin{gathered}
\sup _{s \in[\gamma, v]}\|P(s)-P(\gamma)\|=\sup _{s \in[\gamma, v]}\left\|\bar{x}(s)-\bar{x}(\gamma)-\int_{\gamma}^{s} D F(\bar{x}(\tau), t)\right\|_{\infty} \leq \\
\leq \sup _{s \in[\gamma, v]}\left\|\bar{x}(s)-\int_{\gamma}^{s} D F(\bar{x}(\tau), t)\right\|_{\infty}+\|\bar{x}(\gamma)\|_{\infty}<2 \delta .
\end{gathered}
$$

Também, segue diretamente da definição da função $P$ que $P \in G^{-}([\gamma, v], X)$. Assim, a estabilidade com respeito a perturbações implica $\|\bar{x}(t)\|=\left\|\bar{x}\left(t, \gamma, x_{0}\right)\right\|<\varepsilon$, para todo $t \in[\gamma, v]$, o que implica que a solução $x \equiv 0$ de (3.17) é regularmente estável.

Agora, vamos mostrar o item (ii). Suponha, primeiramente, que a solução $x \equiv 0$ de (3.17) seja regularmente atratora. Então existe um $\widetilde{\delta}>0$ e para todo $\varepsilon>0$, existem um $T=T(\varepsilon) \geq 0$ e um $\rho=\rho(\varepsilon)>0$ tais que, se $\bar{x}:[\gamma, v] \rightarrow B_{c}, t_{0} \leq \gamma<v<+\infty$, for uma função regrada e contínua à esquerda em $[\gamma, v]$ tal que $\|\bar{x}(\gamma)\|<\widetilde{\delta}$ e

$$
\sup _{s \in[\gamma, v]}\left\|\bar{x}(s)-\int_{\gamma}^{s} D F(\bar{x}(\tau), t)\right\|_{\infty}<\rho,
$$

então

$$
\|\bar{x}(t)\|<\varepsilon, \quad t \in[\gamma, v] \cap[\gamma+T,+\infty), \gamma \geq t_{0}
$$

Denotamos a solução da equação diferencial ordinária generalizada perturbada (3.18) satisfazendo $x\left(\gamma, \gamma, x_{0}\right)=x_{0}$ por $x(t)=x\left(t, \gamma, x_{0}\right)$. Suponha que $P \in G^{-}([\gamma, v], X)$. Mais ainda, suponha que exista um $\widetilde{\delta}>0$ e, para todo $\varepsilon>0$, exista um $\rho=\rho(\varepsilon)>0$ tal que $\left\|x_{0}\right\|<\widetilde{\delta} \mathrm{e}$

$$
\sup _{s \in[\gamma, v]}\|P(s)-P(\gamma)\|<\rho .
$$

Então $\left\|x_{0}\right\|=\|x(\gamma)\|<\widetilde{\delta}$ e pela definição de solução da equação (3.18), temos

$$
\sup _{s \in[\gamma, v]}\left\|x(s)-x(\gamma)-\int_{\gamma}^{s} D F(x(\tau), t)\right\|_{\infty}=\sup _{s \in[\gamma, v]}\|P(s)-P(\gamma)\| .
$$


Portanto,

$$
\sup _{s \in[\gamma, v]}\left\|x(s)-\int_{\gamma}^{s} D F(x(\tau), t)\right\|_{\infty}-\|x(\gamma)\|_{\infty} \leq \sup _{s \in[\gamma, v]}\|P(s)-P(\gamma)\|,
$$

o que implica que

$$
\sup _{s \in[\gamma, v]}\left\|x(s)-\int_{\gamma}^{s} D F(x(\tau), t)\right\|_{\infty} \leq \sup _{s \in[\gamma, v]}\|P(s)-P(\gamma)\|+\|x(\gamma)\|_{\infty}<\rho+\widetilde{\delta} .
$$

Assim, segue imediatamente do fato que $x$ é regularmente atratora, que

$$
\left\|x\left(t, \gamma, x_{0}\right)\right\|=\|x(t)\|<\varepsilon, \text { para todo } t \geq \gamma+T, t \in[\gamma, v],
$$

ou seja, a solução $x \equiv 0$ de (3.17) é atratora com respeito a perturbações.

Reciprocamente, suponha que a solução $x \equiv 0$ de (3.17) seja atratora com respeito a perturbações e seja $\bar{x}:[\gamma, v] \rightarrow B_{c}, t_{0} \leq \gamma<v<+\infty$, uma função regrada e contínua à esquerda em $[\gamma, v]$ satisfazendo

$$
\|\bar{x}(\gamma)\|<\widetilde{\delta} \quad \text { e } \sup _{s \in[\gamma, v]}\left\|\bar{x}(s)-\int_{\gamma}^{s} D F(\bar{x}(\tau), t)\right\|_{\infty}<\rho .
$$

Novamente, para $s \in[\gamma, v]$, seja $P(s)-P(\gamma)=\bar{x}(s)-\bar{x}(\gamma)-\int_{\gamma}^{s} D F(\bar{x}(\tau), t)$. Então $\bar{x}(t)$ é uma solução de (3.18) em $[\gamma, v]$. Também temos $P \in G^{-}([\gamma, v], X)$ e, da definição de solução e pela equação (3.21), vale

$$
\sup _{s \in[\gamma, v]}\|P(s)-P(\gamma)\|<\rho+\widetilde{\delta} .
$$

Portanto, a Definição 3.17 nos leva a

$$
\|\bar{x}(t)\|<\varepsilon, \quad t \in[\gamma, v] \cap[\gamma+T,+\infty), \gamma \geq t_{0} .
$$

o que implica a atratividade regular da solução trivial de (3.17).

O item (iii) segue combinando os itens (i) e (ii) e encerramos a demonstração.

Com as definições dadas anteriormente, agora somos capazes de demonstrar um resultado que estabelece uma relação entre as definições de estabilidade para EDOGs e as definições correspondentes para EDFs em medida. Para obter tais resultados, utilizamos a correspondência entre estas equações que foi descrita no Teorema 3.11. 
Relembramos que estamos considerando uma EDF em medida dada por

$$
D x=f\left(x_{t}, t\right) D g
$$

onde $f: S \times\left[t_{0},+\infty\right) \rightarrow \mathbb{R}^{n}$ satisfaz as condições $\left(H_{1}\right),\left(H_{2}\right)$ e $\left(H_{3}\right)$ e $g:\left[t_{0},+\infty\right) \rightarrow \mathbb{R}$ é uma função não decrescente. Mais ainda, estamos assumindo $f(0, t)=0$ para todo $t \in\left[t_{0},+\infty\right)$, o que implica que $y \equiv 0$ é uma solução (3.22).

Também, considere a função

$$
F: O \times\left[t_{0},+\infty\right) \rightarrow G\left(\left[t_{0}-r,+\infty\right), \mathbb{R}^{n}\right)
$$

definida por (3.4), para todo $y \in O$ e $t \in\left[t_{0},+\infty\right)$. Como estamos assumindo $f(0, t)=$ 0 , para todo $t \in\left[t_{0}, \infty\right)$, então, por $(3.4), F(0, t)=0$, para todo $t \in\left[t_{0},+\infty\right)$, o que implica que $x \equiv 0$ é uma solução da EDOG

$$
\frac{d x}{d \tau}=D F(x, t)
$$

Vimos, na seção anterior, que existe uma correspondência biunívoca entre as soluções de (3.22) com condição inicial $y_{t_{0}}=\phi$ e as soluções de (3.23) com condição inicial

$$
x\left(t_{0}\right)(\vartheta)=\left\{\begin{array}{l}
\phi\left(\vartheta-t_{0}\right), t_{0}-r \leq \vartheta \leq t_{0}, \\
\phi(0), t_{0} \leq \vartheta<\infty .
\end{array}\right.
$$

Também estamos considerando a equação perturbada

$$
D y=f\left(y_{t}, t\right) D g+p(t) D u
$$

com $f: S \times\left[t_{0}, \infty\right) \rightarrow \mathbb{R}^{n}$ satisfazendo as mesmas condições de antes, a saber, $\left(H_{1}\right)$, $\left(H_{2}\right)$ e $\left(H_{3}\right)$, e $p:\left[t_{0}, \infty\right) \rightarrow \mathbb{R}^{n}$ satisfazendo as condições $\left(H_{4}\right)$ e $\left(H_{5}\right)$. Pelo Teorema 3.11, a EDOG correspondente à equação (3.25) com condição inicial $y_{t_{0}}=\phi$ é dada por

$$
\frac{d x}{d \tau}=D[F(x, t)+P(t)]
$$

satisfazendo a condição inicial (3.24), onde $F: O \times\left[t_{0},+\infty\right) \rightarrow G\left(\left[t_{0},+\infty\right), \mathbb{R}^{n}\right)$ e $P:\left[t_{0},+\infty\right) \rightarrow G\left(\left[t_{0}-r,+\infty\right), \mathbb{R}^{n}\right)$ são dadas por (3.4) e (3.5) respectivamente.

O teorema a seguir relaciona as definições de estabilidade e atratividade da solução trivial da EDF em medida (3.22) e da solução trivial de sua EDOG correspondente (3.23). Para demonstrá-lo, vamos precisar das equações perturbadas (3.25) e (3.26). 
Teorema 3.20. As seguintes afirmações são válidas.

(i) A solução $y \equiv 0$ de (3.22) será integralmente estável se, e somente se, a solução $x \equiv 0$ de (3.23) for regularmente estável.

(ii) Se a solução $x \equiv 0$ de (3.23) for regularmente atratora, então a solução $y \equiv 0$ de (3.22) será integralmente atratora.

(iii) Se a solução $x \equiv 0$ de (3.23) for regularmente assintoticamente estável, então a solução $y \equiv 0$ de (3.22) será integralmente assintoticamente estável.

Demonstração: Começamos mostrando (i). Suponha que a solução trivial de (3.22) em $[\gamma, v] \subset\left[t_{0},+\infty\right)$ seja integralmente estável. Então, dado $\varepsilon>0$, existe $\delta=\delta(\varepsilon)>0$ tal que se $\phi \in S$ e $p:[\gamma, v] \rightarrow \mathbb{R}^{n}$ forem tais que

$$
\|\phi\|_{\infty}<\delta \text { e } \sup _{t \in[\gamma, v]}\left|\int_{\gamma}^{t} p(s) d u(s)\right|<\delta,
$$

então

$$
|\bar{y}(t ; \gamma, \phi)|<\frac{\varepsilon}{2}, \quad t \in[\gamma, v]
$$

onde $\bar{y}(t ; \gamma, \phi)$ é uma solução de (3.25) tal que $\bar{y}_{\gamma}=\phi$.

Vamos mostrar que a solução trivial da EDOG (3.23), com $F$ dada por (3.4), é estável com respeito a perturbações e, então, o resultado seguirá pela Proposição 3.19.

Seja $x$ uma solução da EDOG perturbada (3.26) com condição inicial $x\left(t_{0}\right)=x_{0}$ dada por (3.24), onde $F$ e $P$ são dadas por (3.4) e (3.5) respectivamente. Seja $\varepsilon>0$ e suponha que exista um $\delta=\delta(\varepsilon)>0$ tal que $\delta<\varepsilon / 2$ e, mais ainda, suponha que

$$
\left\|x_{0}\right\|_{\infty}<\delta \quad \text { e } \sup _{s \in[\gamma, v]}\|P(s)-P(\gamma)\|<\frac{\delta}{2}
$$

onde $x_{0} \in G^{-}\left(\left[t_{0}-r, \infty\right), \mathbb{R}^{n}\right)$ e $P \in G^{-}\left([\gamma, v], \mathbb{R}^{n}\right)$.

Como $\left\|x\left(t_{0}\right)\right\|_{\infty}=\left\|x_{0}\right\|_{\infty}<\delta$, então $\sup _{\theta \in\left[t_{0}-r,+\infty\right)}\left|x\left(t_{0}\right)(\theta)\right|<\delta \mathrm{e}$, portanto, $\sup _{\theta \in\left[t_{0}-r, t_{0}\right]}\left|\phi\left(\theta-t_{0}\right)\right|<\delta$. Assim,

$$
\|\phi\|_{\infty}<\delta
$$

Como $x$ é uma solução da EDOG perturbada, temos

$$
x(u)-x(w)=\int_{w}^{u} D F(x(\tau), t)+P(u)-P(w),
$$


para $u, w \in[\gamma, v]$. Logo

$$
\sup _{s \in[\gamma, v]}\left\|x(s)-x(\gamma)-\int_{\gamma}^{s} D F(x(\tau), t)\right\|=\sup _{s \in[\gamma, v]}\|P(s)-P(\gamma)\|_{\infty}<\delta / 2 .
$$

Pela definição de $P$, obtemos

$$
\begin{aligned}
\|P(s)-P(\gamma)\|_{\infty} & =\sup _{\vartheta \in\left[t_{0}-r, s\right]}|P(s)(\vartheta)-P(\gamma)(\vartheta)| \\
& \geq \sup _{\vartheta \in[\gamma, s]}|P(s)(\vartheta)-P(\gamma)(\vartheta)| \\
& =\sup _{\vartheta \in[\gamma, s]}\left|\int_{\gamma}^{\vartheta} p(w) d u(w)\right| \\
& \geq\left|\int_{\gamma}^{s} p(w) d u(w)\right|
\end{aligned}
$$

Assim, por (3.27) e do fato de que $\sup _{s \in[\gamma, v]}\|P(s)-P(\gamma)\|_{\infty}<\delta / 2$, obtemos

$$
\sup _{s \in[\gamma, v]}\left|\int_{\gamma}^{s} p(v) d u(v)\right|<\delta / 2
$$

Então, pela estabilidade integral da solução trivial de (3.22), $|y(t)|<\varepsilon / 2$, para todo $t \in[\gamma, v]$.

Finalmente, temos

$$
\begin{aligned}
\|x(t)\|_{\infty} & =\sup _{\theta \in\left[t_{0}-r,+\infty\right)}|x(t)(\theta)|=\sup _{\theta \in\left[t_{0}-r, t\right]}|y(\theta)| \\
& \leq\|\phi\|_{\infty}+\sup _{\theta \in\left[t_{0}, t\right]}|y(\theta)| \leq \delta+\frac{\varepsilon}{2}<\varepsilon
\end{aligned}
$$

e segue a suficiência do item (i).

Agora, utilizando (i) da Proposição 3.19, suponhamos que a solução trivial de (3.23) seja estável com respeito a perturbações. Assim, dado $\varepsilon>0$, seja $\delta=\delta(\varepsilon)>0$ da Definição 3.16 .

Seja $y$ uma solução da EDF em medida perturbada (3.25) com $y_{\gamma}=\phi$. Suponha que $\phi \in S,\|\phi\|_{\infty}<\delta$ com $\sup _{s \in[\gamma, v]}\left|\int_{\gamma}^{s} p(v) d u(v)\right|<\delta / 2$. Queremos mostrar que $y \equiv 0$ é integralmente estável, ou seja, $|\bar{y}(t)|<\varepsilon, t \in[\gamma, v]$.

Seja $x$ uma solução da EDOG perturbada (3.26) com $F$ dada por (3.4) e $P$ dada por (3.5), ou seja, $x$ é a solução correspondente a $\bar{y}$ obtida de acordo com o Teorema 3.11 . 
Pela definição da função $P$ e do fato de que $\sup _{s \in[\gamma, v]}\left|\int_{\gamma}^{s} p(w) d u(w)\right|<\delta / 2$, obtemos

$$
\sup _{s \in[\gamma, v]}\|P(s)-P(\gamma)\|_{\infty}<\delta / 2
$$

Assim, da estabilidade com respeito a perturbações da solução trivial de (3.26), obtemos $\|x(t)\|<\varepsilon, t \in[\gamma, v]$, o que implica que, para cada $t \in[\gamma, v]$,

$$
\sup _{\theta \in[\gamma-r, v]}|x(t)(\theta)|<\varepsilon
$$

Portanto, a relação dada no Teorema 3.11 implica

$$
\sup _{\theta \in[\gamma-r, t]}|y(\theta)|<\varepsilon, \quad t \in[\gamma, v]
$$

Em particular,

$$
\sup _{\theta \in[\gamma, v]}|y(\theta)| \leq \sup _{\theta \in[\gamma-r, v]}|y(\theta)|<\varepsilon
$$

Agora, vamos mostrar (ii). Suponha, então, que a solução trivial da EDOG (3.23) seja atratora com respeito a perturbações. Então, existe $\widetilde{\delta}>0$ e dado $\varepsilon>0$ sejam $T=T(\varepsilon) \geq 0$ e $\rho=\rho(\varepsilon)>0$ da Definição 3.17.

Seja $y(t ; \gamma, \phi)$ uma solução da EDF em medida perturbada (3.25). Suponha que

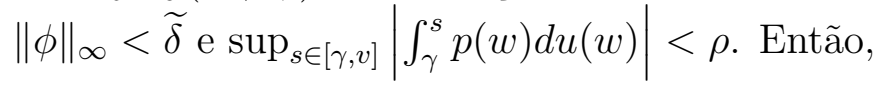

$$
\sup _{s \in[\gamma, v]}\|P(s)-P(\gamma)\|_{\infty}<\rho
$$

Pelo Teorema 3.11, $\left\|x_{0}\right\|_{\infty}=\|\phi\|_{\infty}<\widetilde{\delta}$. Portanto, pela atratividade com respeito a perturbações da solução trivial de (3.23), temos

$$
\|x(t)\|_{\infty}=\left\|x\left(t ; \gamma, x_{0}\right)\right\|_{\infty}<\varepsilon, \quad t \geq \gamma+T, t \in[[\gamma, v]
$$

Logo, para $t \geq \gamma+T, t \in[\gamma, v]$, temos pelo Teorema 3.11,

$$
|y(t)|=|x(t)(t)| \leq\|x(t)\|_{\infty}<\varepsilon .
$$

A afirmação (iii) segue combinando (i), (ii) e a Proposição 3.19. 



\begin{tabular}{|l|l|}
\hline Capítulo \\
\hline
\end{tabular}

\section{Estabilidade de Lyapunov}

Neste capítulo, vamos definir o conceito de estabilidade de Lyapunov para EDOGs e relacioná-lo com a estabilidade no sentido de Lyapunov para EDFs em medida. Ressaltamos que tal definição para EDOGs não existia na literatura.

Os conceitos de estabilidade para EDOGs conhecidos até hoje eram os que envolvem o fato da solução ser de variação limitada, ou no caso de EDOGs relacionadas com EDFs em medida, o fato da solução ser regrada, uma vez que funções de variação limitada são regradas. As noções de estabilidade correspondentes para EDFs, levam a ideias mais específicas do que a noção clássica de estabilidade no sentido de Lyapunov, estabilidade uniforme e estabilidade assintótica.

Pediremos menos hipóteses para os funcionais de Lyapunov do que o que foi assumido no artigo recente [4] e obteremos resultados similares. Além disso, apresentaremos os mesmos resultados (com as mesmas hipóteses) de [4], porém para equações em medida, o que é uma generalização de tais resultados, já que em [4] temos apenas as aplicações para equações diferenciais funcionais retardadas (EDFRs). 


\subsection{Estabilidade de Lyapunov para EDOGs}

Nesta seção, definiremos os conceitos de estabilidade de Lyapunov para EDOGs e mostraremos em quais condições podemos obter esta estabilidade, através de funcionais de Lyapunov.

Lembramos que $X$ denota um espaço de Banach com norma $\|\cdot\|$. Sejam $O \subset X$ um aberto e $F: O \times\left[t_{0},+\infty\right) \rightarrow X$. Consideraremos a EDOG

$$
\frac{d x}{d \tau}=D F(x(\tau), t)
$$

onde vamos supor que $F(0, t)-F(0, s)=0$ para $t, s \geq t_{0}$. Então, para todo intervalo $[\gamma, v] \subset\left[t_{0},+\infty\right)$, temos

$$
\int_{\gamma}^{v} D F(0, t)=F(0, v)-F(0, \gamma)=0
$$

e, portanto, $x \equiv 0$ é uma solução de $(4.1)$ em $\left[t_{0},+\infty\right)$.

A seguir, apresentaremos novos conceitos de estabilidade da solução trivial de uma EDOG.

Definição 4.1. A solução trivial $x \equiv 0$ de (4.1) será dita

(i) Estável (no sentido de Lyapunov), se para quaisquer $\gamma \geq t_{0}$ e $\varepsilon>0$, existir $\delta=\delta(\varepsilon, \gamma)>0$ tal que se $\tilde{x} \in O$ e $\bar{x}:[\gamma, v] \rightarrow O$ for uma solução de (4.1) em $[\gamma, v] \subset\left[t_{0},+\infty\right)$ satisfazendo a condição inicial $\bar{x}(\gamma)=\tilde{x}$ e

$$
\|\tilde{x}\|<\delta
$$

então

$$
\|\bar{x}(t)\|<\varepsilon, \quad t \in[\gamma, v] .
$$

(ii) Uniformemente estável, se o número $\delta$ no item (i) for independente de $\gamma$.

(iii) Uniformemente assintoticamente estável, se existir $\delta_{0}>0$ e para todo $\varepsilon>0$, existir $T=T(\varepsilon) \geq 0$, tal que $\tilde{x} \in O$ e $\bar{x}:[\gamma, v] \rightarrow O$ for uma solução de (4.1) em $[\gamma, v]$ satisfazendo a condição inicial $\bar{x}(\gamma)=\tilde{x} e$

$$
\|\tilde{x}\|<\delta_{0}
$$


então

$$
\|\bar{x}(t)\|<\varepsilon, \quad t \in[\gamma, v] \cap[\gamma+T,+\infty) .
$$

A seguir, enunciaremos uma ferramenta importante para a demonstração de nosso primeiro resultado. Uma demonstração desta proposição pode ser encontrada em [25], Proposição 10.11.

Proposição 4.2. Suponha que $-\infty<a<b<+\infty$ e que $f, g:[a, b] \rightarrow \mathbb{R}$ sejam funções contínuas à esquerda em $(a, b]$. Se para todo $\sigma \in[a, b]$, existir $\delta(\sigma)>0$ tal que para todo $\eta \in(0, \delta(\sigma))$ a desigualdade

$$
f(\sigma+\eta)-f(\sigma) \leq g(\sigma+\eta)-g(\sigma)
$$

vale, então teremos

$$
f(s)-f(a) \leq g(s)-g(a)
$$

para todo $s \in[a, b]$.

O lema a seguir será essencial na demonstração dos teoremas de estabilidade para EDOGs. Ele é uma adaptação de [3], Lema 5.2 e do Lema 10.12 de [25].

Lema 4.3. Suponha que $V:\left[t_{0},+\infty\right) \times X \rightarrow \mathbb{R}$ seja tal que $V(\cdot, x):\left[t_{0},+\infty\right) \rightarrow \mathbb{R}$ é contínua à esquerda em $\left(t_{0},+\infty\right)$ para $x \in X$ e que exista uma função $\phi: X \rightarrow \mathbb{R}$ tal que para toda função $x:[\gamma, v] \rightarrow X, \operatorname{com}[\gamma, v] \subset\left[t_{0},+\infty\right)$, contínua à esquerda $e$ regrada, temos

$$
\dot{V}(t, x(t))=\limsup _{\eta \rightarrow 0^{+}} \frac{V(t+\eta, x(t+\eta))-V(t, x(t))}{\eta} \leq \phi(x(t)), \quad t \in[\gamma, v] .
$$

Então,

$$
V(v, x(v))-V(\gamma, x(\gamma)) \leq M(v-\gamma)
$$

onde $M=\sup _{t \in[\gamma, v]} \phi(x(t))$

Demonstração: Seja $\sigma \in[\gamma, v]$ qualquer. Pela condição $(4.2)$, existe $\delta(\sigma)>0$ suficientemente pequeno tal que, para todo $\rho \in(0, \delta(\sigma))$ vale que

$$
V(\sigma+\rho, x(\sigma+\rho))-V(\sigma, x(\sigma)) \leq \rho \phi((x(\sigma))) \leq M \rho
$$

onde $M=\sup _{t \in[\gamma, v]} \phi(x(t))$. 
Definindo $f(t)=M t$, obtemos

$$
V(\sigma+\rho, x(\sigma+\rho))-V(\sigma, x(\sigma)) \leq f(\sigma+\rho)-f(\sigma)
$$

Estamos, então, nas hipóteses do Proposição 4.2 e, portanto,

$$
V(v, x(v))-V(\gamma, x(\gamma)) \leq f(v)-f(\gamma)=M(v-\gamma)
$$

o que conclui a demonstração.

A seguir, definiremos funcional de Lyapunov para EDOGs. Esta definição foi dada em [4] e é mais geral do que a definição dada em [25].

Definição 4.4. Diremos que $V:\left[t_{0},+\infty\right) \times X \rightarrow \mathbb{R}$ é um funcional de Lyapunov (com respeito à EDOG (4.1)), se as seguintes condições forem satisfeitas:

(i) $V(\cdot, x):\left[t_{0},+\infty\right) \rightarrow \mathbb{R}$ é contínua à esquerda em $\left(t_{0},+\infty\right)$, para todo $x \in X$;

(ii) Existe uma função $b: \mathbb{R}^{+} \rightarrow \mathbb{R}^{+}$, contínua, estritamente crescente, com $b(0)=0$ (diremos que tal função é de classe Hahn), tal que

$$
V(t, x) \geq b(\|x\|)
$$

para quaisquer $t \in\left[t_{0},+\infty\right)$ e $x \in X$;

(iii) Para toda $\bar{x}:[\gamma, v] \rightarrow X$ solução de (4.1), com $[\gamma, v] \subset\left[t_{0},+\infty\right)$, temos

$$
\dot{V}(t, \bar{x}(t))=\limsup _{\eta \rightarrow 0^{+}} \frac{V(t+\eta, \bar{x}(t+\eta))-V(t, \bar{x}(t))}{\eta} \leq 0, \quad t \in[\gamma, v]
$$

ou seja, a derivada de $V$ é não positiva ao longo de soluções de (4.1).

O teorema a seguir nos garante a estabilidade uniforme da solução trivial de (4.1).

Teorema 4.5. Suponha que $V:\left[t_{0},+\infty\right) \times \bar{B}_{\rho} \rightarrow \mathbb{R}$ seja um funcional de Lyapunov para a EDOG (4.1), onde $\bar{B}_{\rho}=\{x \in X ;\|x\| \leq \rho\}$. Suponha, ainda, que $V$ satisfaça a seguinte condição:

- Existe uma constante $K>0$ tal que

$$
|V(\gamma, \bar{x}(\gamma))| \leq K\|\bar{x}(\gamma)\|
$$

para toda solução $\bar{x}:[\gamma, v] \rightarrow X$ de (4.1) em $[\gamma, v] \subset\left[t_{0},+\infty\right)$. 
Então a solução trivial $x \equiv 0$ da EDOG (4.1) será uniformemente estável.

Demonstração: Seja $\bar{x}:[\gamma, v] \rightarrow X$ uma solução da EDOG (4.1) em $[\gamma, v] \subset$ $\left[t_{0},+\infty\right.$ ). Pela condição (iii) da Definição 4.4 e pelo Lema 4.3,

$$
V(t, \bar{x}(t)) \leq V(\gamma, \bar{x}(\gamma)), \quad t \in[\gamma, v]
$$

Como $V$ é um funcional de Lyapunov, existe uma função $b: \mathbb{R}^{+} \rightarrow \mathbb{R}^{+}$de classe Hahn tal que $V(t, x) \geq b(\|x\|)$, para qualquer par $(t, x) \in\left[t_{0},+\infty\right) \times \bar{B}_{\rho}$.

Dado $\varepsilon>0$, temos $b(\varepsilon)>0$. Seja $\delta(\varepsilon)>0$ tal que $K \delta(\varepsilon)<b(\varepsilon)$. Se $\|\bar{x}(\gamma)\|<\delta(\varepsilon)$, obtemos

$$
\begin{aligned}
V(t, \bar{x}(t)) \leq V(\gamma, \bar{x}(\gamma)) & \leq|V(\gamma, \bar{x}(\gamma))| \\
& \leq K\|\bar{x}(\gamma)\|<K \delta(\varepsilon)<b(\varepsilon), \quad t \in[\gamma, v]
\end{aligned}
$$

Por outro lado, suponha que exista $u \in[\gamma, v]$ tal que $\|\bar{x}(u)\| \geq \varepsilon$. Então

$$
V(u, \bar{x}(u)) \geq b(\|\bar{x}(u)\|) \geq b(\varepsilon),
$$

o que contradiz (4.4). Portanto $\|\bar{x}(t)\|<\varepsilon$, para todo $t \in[\gamma, v]$ necessariamente e a demonstração está completa.

Teorema 4.6. Seja $V:\left[t_{0},+\infty\right) \times \bar{B}_{\rho} \rightarrow \mathbb{R}$ um funcional de Lyapunov, onde $\bar{B}_{\rho}=$ $\{x \in X ;\|x\| \leq \rho\}$. Suponha que $V$ satisfaça a condição (i) do Teorema 4.5. Suponha, ainda, que exista uma função contínua $\phi: X \rightarrow \mathbb{R}$, com $\phi(0)=0$ e $\phi(x)>0$ para $x \neq 0$, tal que para toda solução $\bar{x}:[\gamma, v] \rightarrow \bar{B}_{\rho}$ da equação (4.1), com $[\gamma, v] \subset\left[t_{0},+\infty\right)$, vale

$$
\dot{V}(t, \bar{x}(t)) \leq-\phi(\bar{x}(t)), \quad t \in[\gamma, v] .
$$

Então a solução trivial $x \equiv 0$ da EDOG (4.1) será uniformemente assintoticamente estável.

Demonstração: Como todas as condições do Teorema 4.5 estão satisfeitas, a solução $x \equiv 0$ é uniformemente estável. Sabemos, então, que:

(I) Existe $\tilde{\delta} \in(0, \rho)$ tal que se $\bar{x}:[\gamma, v] \rightarrow X$ for uma solução de $(4.1)$ em $[\gamma, v] \subset$ $\left[t_{0},+\infty\right) \mathrm{com}$

$$
\|\bar{x}(\gamma)\|<\tilde{\delta}
$$


então

$$
\|\bar{x}(t)\|<\rho, \quad t \in[\gamma, v] .
$$

(II) Para todo $\varepsilon>0$, existe $\delta=\delta(\varepsilon)>0$, com $\delta<\varepsilon$, tal que se $\bar{x}:[\bar{\gamma}, \bar{v}] \rightarrow B_{c}$ for uma solução de $(4.1)$ em $[\bar{\gamma}, \bar{v}] \subset\left[t_{0},+\infty\right)$ com

$$
\|\bar{x}(\bar{\gamma})\|<\delta
$$

então

$$
\|\bar{x}(t)\|<\varepsilon, \quad t \in[\bar{\gamma}, \bar{v}] .
$$

Seja

$$
\begin{aligned}
N & =\sup \{-\phi(y) ; \delta(\varepsilon) \leq\|y\|<\varepsilon\} \\
& =-\inf \{\phi(y) ; \delta(\varepsilon) \leq\|y\|<\varepsilon\}<0
\end{aligned}
$$

e definimos

$$
T(\varepsilon):=\min \left\{v-\gamma,-K \frac{\tilde{\delta}}{N}\right\}>0,
$$

onde $K$ vem de (i) do Teorema 4.5.

Suponha que $\bar{x}:[\gamma, v] \rightarrow B_{c}$ seja uma solução da EDOG (4.1) em $[\gamma, v] \subset\left[t_{0},+\infty\right)$ tal que

$$
\|\bar{x}(\gamma)\|<\tilde{\delta} .
$$

Queremos mostrar que

$$
\|\bar{x}(t)\|<\varepsilon, \quad t \in[\gamma, v] \cap[\gamma+T(\varepsilon),+\infty), \gamma \geq 0 .
$$

Afirmamos que existe $t^{*} \in[\gamma, \gamma+T(\varepsilon)]$ tal que $\left\|\bar{x}\left(t^{*}\right)\right\|<\delta(\varepsilon)$. De fato, suponha o contrário, ou seja, $\|\bar{x}(s)\| \geq \delta(\varepsilon)$, para todo $s \in[\gamma, \gamma+T(\varepsilon)]$. Pelo Lema 4.3, pela condição (i) do Teorema 4.5 e por (4.6), temos

$$
\begin{aligned}
V(\gamma+T(\varepsilon), \bar{x}(\gamma+T(\varepsilon))) & \leq V(\gamma, \bar{x}(\gamma))+N \cdot T(\varepsilon) \\
& \leq K\|\bar{x}(\gamma)\|+N\left(-K \frac{\tilde{\delta}}{N}\right) \\
& <K \tilde{\delta}-K \tilde{\delta}=0 .
\end{aligned}
$$


Por outro lado, como $V$ é um funcional de Lyapunov, existe uma função $b: \mathbb{R}^{+} \rightarrow$ $\mathbb{R}^{+}$de classe Hahn tal que

$$
V(t, x) \geq b(\|x\|), \quad(t, x) \in\left[t_{0},+\infty\right) \times X .
$$

Assim,

$$
V(\gamma+T(\varepsilon), \bar{x}(\gamma+T(\varepsilon))) \geq b(\|\bar{x}(\gamma+T(\varepsilon))\|) \geq b(\delta(\varepsilon))>0,
$$

o que é uma contradição. Logo, $\|\bar{x}(t)\|<\varepsilon$ para $t \in\left[t^{*}, v\right]$, uma vez que (II) vale para $\bar{\gamma}=t^{*}$ e $\bar{v}=v$. Além disso, $\|\bar{x}(t)\|<\varepsilon$ para $t>\gamma+T(\varepsilon)$, já que $t^{*} \in[\gamma, \gamma+T(\varepsilon)]$, o que conclui o resultado.

\subsection{Estabilidade de Lyapunov para EDFs em medida via EDOGs}

Nesta seção, vamos considerar a EDF em medida

$$
D y=f\left(y_{t}, t\right) D g
$$

com $f: S \times\left[t_{0},+\infty\right) \rightarrow \mathbb{R}^{n}$, onde $S=\left\{x_{t} ; x \in O, t \in\left[t_{0},+\infty\right)\right\}$ e $O \subset G\left(\left[t_{0}-\right.\right.$ $r,+\infty), \mathbb{R}^{n}$ ) tem a propriedade do prolongamento definida no Capítulo 3.

Também vamos considerar $g:\left[t_{0},+\infty\right) \rightarrow \mathbb{R}$ não decrescente e $f(0, t)=0$ para todo $t \in\left[t_{0},+\infty\right)$. Assim $y \equiv 0$ é uma solução de (4.7).

Suponhamos que $f$ satisfaça as condições $\left(H_{1}\right)$ até $\left(H_{3}\right)$ do Capítulo 3, ou seja,

$\left(H_{1}\right)$ A integral de Kurzweil-Henstock-Stieltjes $\int_{t_{0}}^{t} f\left(y_{s}, s\right) d g(s)$ existe para quaisquer $y \in O$ e $t \in\left[t_{0}, \infty\right)$.

$\left(H_{2}\right)$ Existe uma função $M:\left[t_{0}, \infty\right) \rightarrow \mathbb{R}$ localmente Lebesgue-Stieltjes integrável, com respeito a $g$ tal que a desigualdade

$$
\left|\int_{u}^{v} f\left(y_{s}, s\right) d g(s)\right| \leq \int_{u}^{v} M(s) d g(s)
$$

vale para quaisquer $y \in O$ e $u, v \in\left[t_{0}, \infty\right)$. 
$\left(H_{3}\right)$ Existe uma função $L:\left[t_{0}, \infty\right) \rightarrow \mathbb{R}$ localmente Lebesgue-Stieltjes integrável com respeito a $g$ tal que a desigualdade

$$
\left|\int_{u}^{v}\left[f\left(y_{s}, s\right)-f\left(z_{s}, s\right)\right] d g(s)\right| \leq \int_{u}^{v} L(s)\left\|y_{s}-z_{s}\right\|_{\infty} d g(s)
$$

vale para quaisquer $y, z \in O$ e $u, v \in\left[t_{0}, \infty\right)$.

Além disso, assumimos que $F$ da equação (4.1) é dada pela relação descrita na equação (3.4), ou seja, para $y \in O$ e $t \in\left[t_{0},+\infty\right)$, temos

$$
F(y, t)(\vartheta)=\left\{\begin{array}{l}
0, \quad t_{0}-r \leq \vartheta \leq t_{0}, \\
\int_{t_{0}}^{\vartheta} f\left(y_{s}, s\right) d g(s), \quad t_{0} \leq \vartheta \leq t<+\infty, \\
\int_{t_{0}}^{t} f\left(y_{s}, s\right) d g(s), \quad t \leq \vartheta<+\infty .
\end{array}\right.
$$

Neste caso, $F: O \times\left[t_{0},+\infty\right) \rightarrow G\left(\left[t_{0}-r,+\infty\right), \mathbb{R}^{n}\right)$ e pelo Teorema 3.11, temos uma relação entre problemas de valor inicial para as equações (4.1) e (4.7).

Dados $t \geq t_{0}$ e uma função $\psi \in G^{-}\left([-r, 0], \mathbb{R}^{n}\right)$, consideramos a EDF em medida (4.7) com condição inicial $y_{t}=\psi$. Consideramos, também, que a EDOG (4.1) sujeita à condição inicial $x(t)=\widetilde{x}$, onde $\widetilde{x}(\tau)=\psi(\tau-t), t-r \leq \tau \leq t$, e $\widetilde{x}(\tau)=\psi(0), \tau \geq t$. Pelo Teorema 3.12, existe uma única solução $y:[t-r, v] \rightarrow \mathbb{R}^{n}$ da EDF em medida (4.7) que satisfaz $y_{t}=\psi$. E, pelo Teorema 3.11, podemos encontrar uma solução $x:[t, v] \rightarrow$ $G^{-}\left([t, v], \mathbb{R}^{n}\right)$ da $\operatorname{EDOG}(4.1)$, com condição inicial $x(t)=\widetilde{x}$. Então $x(t)(t+\theta)=y(t+\theta)$ para todo $\theta \in[-r, 0]$ e, portanto, $(x(t))_{t}=y_{t}$. Neste caso, escrevemos $y_{t+\eta}=y_{t+\eta}(t, \psi)$ para todo $\eta \geq 0$. Então, para $U:\left[t_{0},+\infty\right) \times G^{-}\left([-r, 0], \mathbb{R}^{n}\right) \rightarrow \mathbb{R}$, definimos

$$
D^{+} U(t, \psi)=\limsup _{\eta \rightarrow 0+} \frac{U\left(t+\eta, y_{t+\eta}(t, \psi)\right)-U\left(t, y_{t}(t, \psi)\right)}{\eta}, \quad t \geq t_{0} .
$$

Por outro lado, dado $t \geq t_{0}$, se $\widetilde{x} \in G^{-}\left([t-r,+\infty), \mathbb{R}^{n}\right)$ for tal que $\widetilde{x}(\tau)=\psi(\tau-t)$, $t-r \leq \tau \leq t$, e $\widetilde{x}(\tau)=\psi(0), \tau \geq t$, então existirá, pelo Teorema 1.6, uma única solução $x:[t, \bar{v}] \rightarrow G^{-}\left([t, \bar{v}], \mathbb{R}^{n}\right)$ da EDOG (4.1) tal que $x(t)=\widetilde{x}$, com $[t, \bar{v}] \subset\left[t_{0},+\infty\right)$. Pelo Teorema 3.11, podemos encontrar uma solução $y:[t-r, \bar{v}] \rightarrow \mathbb{R}^{n}$ de (4.7) que satisfaz $y_{t}=\psi$ e é descrita em termos de $x$. Neste caso, escrevemos $x_{\psi}(t)$ em lugar de $x(t)$ e temos $y_{t}(t, \psi)=\left(x_{\psi}(t)\right)_{t}=\psi$. Consequentemente, $\left(t, x_{\psi}(t)\right) \mapsto\left(t, y_{t}(t, \psi)\right)$ é uma aplicação biunívoca e podemos definir uma função $V:\left[t_{0},+\infty\right) \times O \rightarrow \mathbb{R}$ por

$$
V\left(t, x_{\psi}(t)\right)=U\left(t, y_{t}(t, \psi)\right)
$$


Portanto

$$
D^{+} U(t, \psi)=\limsup _{\eta \rightarrow 0^{+}} \frac{V\left(t+\eta, x_{\psi}(t+\eta)\right)-V\left(t, x_{\psi}(t)\right)}{\eta}, \quad t \geq t_{0} .
$$

Assim, dado $t \geq t_{0}$, temos $\left\|y_{t}(t, \psi)\right\|=\left\|x_{\psi}(t)\right\|$, pois

$$
\begin{aligned}
\left\|y_{t}(t, \psi)\right\| & =\left\|y_{t}\right\|=\sup _{-r \leq \theta \leq 0}|y(t+\theta)|=\sup _{t-r \leq \tau \leq t}|y(\tau)|=\sup _{t-r \leq \tau \leq t}\left|x_{\psi}(t)(\tau)\right| \\
& =\sup _{t-r \leq \tau<+\infty}\left|x_{\psi}(t)(\tau)\right|=\left\|x_{\psi}(t)\right\|,
\end{aligned}
$$

onde a quarta igualdade segue do Teorema 3.11

A seguir, definimos um funcional de Lyapunov $U:\left[t_{0},+\infty\right) \times G^{-}\left([-r, 0], \mathbb{R}^{n}\right) \rightarrow \mathbb{R}$ com respeito à EDF em medida (4.7).

Definição 4.7. Diremos que $U:\left[t_{0},+\infty\right) \times G^{-}\left([-r, 0], \mathbb{R}^{n}\right) \rightarrow \mathbb{R}$ é um funcional de Lyapunov (com respeito a EDF em medida (4.7)), se as seguintes condições forem satisfeitas:

(i) $U(\cdot, \psi):\left[t_{0},+\infty\right) \rightarrow \mathbb{R}$ é contínua à esquerda em $\left(t_{0},+\infty\right)$, para qualquer $\psi \in$ $G^{-}\left([-r, 0], \mathbb{R}^{n}\right)$

(ii) Existe uma função $b: \mathbb{R}_{+} \rightarrow \mathbb{R}_{+}$de classe Hahn tal que

$$
U(t, \psi) \geq b(\|\psi\|)
$$

para cada $t \geq t_{0}$ e para cada $\psi \in G^{-}\left([-r, 0], \mathbb{R}^{n}\right)$;

(iii) A desigualdade

$$
D^{+} U(t, \psi) \leq 0
$$

vale para cada $t \geq t_{0}$ e para cada $\psi \in G^{-}\left([-r, 0], \mathbb{R}^{n}\right)$.

Note que, se $U:\left[t_{0},+\infty\right) \times G^{-}\left([-r, 0], \mathbb{R}^{n}\right) \rightarrow \mathbb{R}$ for um funcional de Lyapunov, então o funcional $V:\left[t_{0},+\infty\right) \times O \rightarrow \mathbb{R}$ dado pela relação (4.8) satisfará as condições $(i),($ ii $)$ e (iii) da Definição 4.4, ou seja, $V$ também será um funcional de Lyapunov. Por (4.8) e (4.9), é fácil ver que $V$ satisfaz as condições $(i)$ e (iii) da Definição 4.4. Portanto, basta verificarmos que $V$ satisfaz $(i i)$. De fato, se $t \geq t_{0}$ e $z \in O$, então existirão uma solução $x$ da EDOG (4.1) e uma função $\psi \in G^{-}\left([-r, 0], \mathbb{R}^{n}\right)$ tais que $z=x_{\psi}(t)$, $\left(x_{\psi}(t)\right)_{t}=y_{t}(t, \psi)$, onde $y$ é a solução da EDF em medida (4.7) correspondente a $x$. Mas

$$
\|\psi\|=\left\|y_{t}(t, \psi)\right\|=\left\|x_{\psi}(t)\right\|=\|z\| .
$$


Como $U$ é um funcional de Lyapunov, pela condição (ii) da Definição 4.4, obtemos

$$
V(t, z)=V\left(t, x_{\psi}(t)\right)=U\left(t, y_{t}(t, \psi)\right)=U(t, \psi) \geq b(\|\psi\|)=b(\|z\|)
$$

o que mostra (ii).

Para o que vem a seguir, consideremos os conjuntos

$$
\bar{E}_{\rho}=\left\{y \in G^{-}\left([-r, 0], \mathbb{R}^{n}\right) ;\|y\| \leq \rho\right\}
$$

e

$$
\bar{B}_{\rho}=\{x \in O ;\|x\| \leq \rho\},
$$

Teorema 4.8. Considere a equação diferencial funcional em medida (4.7). Suponha que $U:\left[t_{0},+\infty\right) \times \bar{E}_{\rho} \rightarrow \mathbb{R}$ seja um funcional de Lyapunov para a equação (4.7). Suponha, ainda, que para cada $\psi \in \bar{E}_{\rho}$,

$$
|U(t, \psi)| \leq K\|\psi\|
$$

Então a solução $y \equiv 0$ de (4.7) será uniformemente estável.

Demonstração: Como $f$ satisfaz as condições $\left(H_{1}\right)$ até $\left(H_{3}\right)$ do Capítulo 3 , a função $F$ na equação (4.1) pertence à classe $\mathcal{F}(\Omega, h)$, onde $\Omega=O \times\left[t_{0},+\infty\right)$ com $h:\left[t_{0},+\infty\right) \rightarrow \mathbb{R}$ dada por

$$
h(t)=\int_{t_{0}}^{t}[M(s)+L(s)] d g(s), \quad t \in\left[t_{0},+\infty\right) .
$$

Seja $V:\left[t_{0},+\infty\right) \times \bar{B}_{\rho} \rightarrow \mathbb{R}$ dado por (4.8). Como visto acima, $V$ é um funcional de Lyapunov para a EDOG (4.1). Note que a condição (4.10) implica que a condição (i) do Teorema 4.5 é válida. De fato, se denotarmos $\bar{y}(t)=\bar{y}(t ; \gamma, \phi)$ uma solução qualquer de (4.7) e definirmos

$$
\bar{x}(t)(\tau)=\left\{\begin{array}{l}
\bar{y}(\tau), \gamma-r \leq \tau \leq t, \\
\bar{y}(t), \tau \geq t
\end{array}\right.
$$

então, pelo Teorema 3.11, $\bar{x}(t)$ será uma solução da EDOG (4.1), satisfazendo a condição inicial $\bar{x}(\gamma)=\widetilde{x}$, com

$$
\widetilde{x}(\tau)= \begin{cases}\phi(\tau-\gamma), & \gamma-r \leq \tau \leq \gamma \\ \phi(0), & \tau \geq \gamma\end{cases}
$$


Logo,

$$
\begin{aligned}
V\left(\gamma, \bar{x}_{\phi}(\gamma)\right) & =U\left(\gamma, \bar{y}_{\gamma}(t, \phi)\right)=U(\gamma, \phi) \\
& \leq K\|\phi\|=K \sup _{\gamma-r \leq \tau<+\infty}|\widetilde{x}(\tau)|=K\|\bar{x}(\gamma)\|_{\infty}
\end{aligned}
$$

Assim, as hipóteses do Teorema 4.5 estão satisfeitas e a solução $x \equiv 0$ da EDOG (4.1) é uniformemente estável.

Dado $\varepsilon>0$, queremos mostrar que existe $\delta(\varepsilon)>0$ tal que, se $\phi \in \bar{E}_{\rho}$ e $\bar{y}:[\gamma, v] \rightarrow$ $\mathbb{R}^{n}$ for uma solução de (4.7) tal que $\bar{y}_{\gamma}=\phi$, com $t_{0} \leq \gamma<v<\infty$ e

$$
\|\phi\|<\delta
$$

então

$$
\left\|\bar{y}_{t}(\gamma, \phi)\right\|<\varepsilon, \quad t \in[\gamma, v]
$$

Como

$$
\|\bar{x}(\gamma)\|=\sup _{\gamma-r \leq \tau<+\infty}|\widetilde{x}(\tau)|=\|\phi\|<\delta
$$

e $x \equiv 0$ é uniformemente estável, então $\|\bar{x}(t)\|<\varepsilon$, para todo $t \in[\gamma, v]$. Logo (4.11) implica que, para qualquer $t \in[\gamma, v]$,

$$
\begin{aligned}
\left\|\bar{y}_{t}(\gamma, \phi)\right\| & =\left\|\bar{y}_{t}\right\|=\sup _{-r \leq \theta \leq 0}|\bar{y}(t+\theta)| \leq \sup _{\gamma-r \leq \tau \leq v}|\bar{y}(\tau)| \\
& =\sup _{\gamma-r \leq \tau \leq v}|\bar{x}(v)(\tau)|=\sup _{\gamma-r \leq \tau<+\infty}|\bar{x}(v)(\tau)| \\
& =\|\bar{x}(v)\|<\varepsilon .
\end{aligned}
$$

Portanto temos (4.14), o que completa a demonstração.

Teorema 4.9. Considere a equação diferencial funcional em medida (4.7). Suponha que $U:\left[t_{0},+\infty\right) \times \overline{E_{\rho}} \rightarrow \mathbb{R}$ seja um funcional de Lyapunov e que satisfaça a condição (4.10) do Teorema 4.8. Além disso, suponha que exista uma função contínua $\Lambda$ : $\mathbb{R}_{+} \rightarrow \mathbb{R}_{+}$tal que $\Lambda(0)=0$ e $\Lambda(x)>0$ se $x \neq 0$, de forma que, para qualquer $\psi \in \bar{E}_{\rho}$, tenhamos

$$
D^{+} U(t, \psi) \leq-\Lambda(\|\psi\|), \quad t \geq t_{0}
$$

Então a solução trivial y $\equiv 0$ de (4.7) será uniformemente assintoticamente estável.

Demonstração: Seja $V:\left[t_{0},+\infty\right) \times \bar{B}_{\rho} \rightarrow \mathbb{R}$ dado por (4.8). Então as hipóteses do Teorema 4.5 estão satisfeitas (já que $V$ também satisfaz a condição (4.10)). 
Definimos $\Phi: \bar{B}_{\rho} \rightarrow \mathbb{R}$ por $\Phi(z)=\Lambda(\|z\|)$, para $z \in \bar{B}_{\rho}$. Então $\Phi$ é contínua, $\Phi(0)=0$ e $\Phi(z)>0$ sempre que $z \neq 0$.

Se $x:[t,+\infty) \rightarrow \bar{B}_{\rho}$ for uma solução de (4.1) tal que $(x(t))_{t}=\psi$, onde $t \in\left[t_{0},+\infty\right)$ e $\psi \in \bar{E}_{\rho}$, consideramos $y:[t-r,+\infty) \rightarrow \mathbb{R}^{n}$ a solução de (4.7), dada pelo Teorema 3.11, que satisfaz $y_{t}=\psi$. Por (4.17), temos

$$
\begin{gathered}
\limsup _{\eta \rightarrow 0+} \frac{V\left(t+\eta, x_{\psi}(t+\eta)\right)-V\left(t, x_{\psi}(t)\right)}{\eta}=D^{+} U\left(t, y_{t}(t, \psi)\right)= \\
=D^{+} U(t, \psi) \leq-\Lambda(\|\psi\|)=-\Lambda\left(\left\|y_{t}\right\|\right) .
\end{gathered}
$$

Como,

$$
\left\|y_{t}\right\|=\left\|x_{\psi}(t)\right\|
$$

obtemos

$$
\limsup _{\eta \rightarrow 0+} \frac{V\left(t+\eta, x_{\psi}(t+\eta)\right)-V\left(t, x_{\psi}(t)\right)}{\eta} \leq-\Lambda\left(\left\|y_{t}\right\|\right)=-\Lambda\left(\left\|x_{\psi}(t)\right\|\right)=-\Phi\left(x_{\psi}(t)\right)
$$

e as hipóteses do Teorema 4.6 estão satisfeitas. Então $x \equiv 0$ é uniformemente assintoticamente estável e, assim, existe $\delta_{0}>0$ e para todo $\varepsilon>0$, existe $T=T(\varepsilon) \geq 0$ tal que, se $\bar{x}:[\gamma, v] \rightarrow B_{c}, t_{0} \leq \gamma<v<+\infty$, for uma solução de (4.1) em $[\gamma, v]$ tal que

$$
\|\bar{x}(\gamma)\|<\delta_{0}
$$

então

$$
\|\bar{x}(t)\|<\varepsilon, \quad t \in[\gamma, v] \cap[\gamma+T,+\infty), \gamma \geq t_{0} .
$$

Dado $\varepsilon>0$, sejam $\delta_{0}>0$ e $T=T(\varepsilon)$ como acima. Seja $\phi \in E_{c}$ e consideramos $\bar{y}:[\gamma-r,+\infty) \rightarrow \mathbb{R}^{n}$, a solução de $(4.7)$ tal que $\bar{y}_{\gamma}=\phi$ e

$$
\|\phi\|<\delta_{0}
$$

Queremos mostrar que

$$
\left\|\bar{y}_{t}(\gamma, \phi)\right\|<\varepsilon, \quad t \in[\gamma, v] \cap[\gamma+T,+\infty), \gamma \geq t_{0}
$$

Mas este fato segue como na demonstração do Teorema 4.8. Por (4.20), é possível obter (4.18), como feito no Teorema 4.8. Assim, (4.21) segue, já que temos (4.16), como no Teorema 4.8 e (4.19). 
Nos teoremas a seguir, consideraremos funções $U:\left[t_{0}-r,+\infty\right) \times \mathbb{R}^{n} \rightarrow \mathbb{R}$. Tais resultados são mais gerais dos que aqueles apresentados em [4], pois aqui não pedimos a hipótese de que o funcional $U$ satisfaça uma condição de Lipschitz na segunda variável, além, é claro, de aqui tratarmos de EDFs em medida e em [4] ser tratado o caso de uma EDFR usual. Entretanto, as demonstrações são análogas às demonstrações feitas nos Teoremas 4.8 e 4.9 de [4] e, portanto, as demonstrações serão omitidas aqui. A ideia em ambos os teoremas é utilizar o Teorema 4.9. Estes resultados são teoremas do tipo Razumikhin e também são generalizações dos Teoremas 5.4.1 e 5.4.2 de [17].

A derivada de $U$ ao longo das soluções de (4.7) é dada por

$$
D^{+} U(t, y(t))=\limsup _{\eta \rightarrow 0^{+}} \frac{U(t+\eta, y(t+\eta, t, \psi))-U(t, y(t, t, \psi))}{\eta}, \quad t \geq t_{0},
$$

onde $y(s, t, \psi)$ é a solução de $(4.7)$ que satisfaz $y_{t}=\psi, \psi \in G^{-}\left([-r, 0], \mathbb{R}^{n}\right)$. Podemos escrever $D^{+} U(t, \psi(0))$ no lugar de $D^{+} U(t, y(t))$, já que dados $t \geq t_{0}$ e uma função inicial $\psi \in G^{-}\left([-r, 0], \mathbb{R}^{n}\right)$, existe uma única solução de $(4.7)$ que satisfaz $y_{t}=\psi$ e, assim, $y(t)=\psi(0)$. Vamos, então, aos teoremas.

Teorema 4.10. Considere a equação diferencial funcional em medida (4.7). Suponha que $U:\left[t_{0}-r,+\infty\right) \times \mathbb{R}^{n} \rightarrow \mathbb{R}$ seja contínua à esquerda em $\left(t_{0}-r,+\infty\right)$ e que os limites

$$
U(t-, y(t-))=\lim _{s \rightarrow t^{-}} U(s, y(s)), \quad t \in\left(t_{0}-r,+\infty\right)
$$

e

$$
U(t+, y(t+))=\lim _{s \rightarrow t^{+}} U(s, y(s)), \quad t \in\left[t_{0}-r,+\infty\right),
$$

existam, com a igualdade $U(t-, y(t-))=U(t, y(t))$ satisfeita, para $y \in G^{-}\left(\left[t_{0}-\right.\right.$ $\left.r,+\infty), \mathbb{R}^{n}\right)$. Além disso, suponha que $U$ satisfaça as seguintes condições:

(i) Existe uma função $b: \mathbb{R}^{+} \rightarrow \mathbb{R}^{+}$de classe Hahn tal que

$$
U(t, y(t)) \geq b\left(\left\|y_{t}\right\|\right)
$$

para quaisquer $y \in G^{-}\left(\left[t_{0}-r,+\infty\right), \mathbb{R}^{n}\right)$ e $t \in\left[t_{0},+\infty\right)$;

(ii) Existe uma função $\Lambda: \mathbb{R}^{+} \rightarrow \mathbb{R}^{+}$tal que

$$
D^{+} U(t, \psi(0)) \leq-\Lambda(|\psi(0)|), \text { sempre que } U(t+\theta, \psi(\theta)) \leq U(t, \psi(0))
$$

para quaisquer $t \in\left[t_{0},+\infty\right), \theta \in[-r, 0]$ e $\psi \in G^{-}\left([-r, 0], \mathbb{R}^{n}\right)$.

Então a solução trivial $y \equiv 0$ de (4.7) será uniformemente estável. 
A ideia da demonstração do Teorema 4.10 é definir

$$
\bar{U}(s, \xi)=\sup _{\theta \in[-r, 0]} U(s+\theta, \xi(\theta))
$$

para $s \geq t_{0}$ e $\xi \in \bar{E}_{\rho}$ e mostrar que este funcional satisfaz as condições do Teorema 4.8. Como dito anteriormente, o restante da demonstração será omitida por ser análoga à demonstração do Teorema 4.8 de [4].

Antes de enunciarmos o último resultado, vamos definir o conceito de atrator global como feito em [17]. Antes disso, precisamos definir o conceito de processo como em [17]. A definição a seguir é a Definição 4.1.1 de [17].

Definição 4.11. Seja $X$ um espaço de Banach, $\mathbb{R}^{+}=[0,+\infty), u: \mathbb{R} \times X \times \mathbb{R}^{+} \rightarrow X$ uma aplicação dada e defina $U(\sigma, t): X \rightarrow X$ para $\sigma \in \mathbb{R}, t \in \mathbb{R}^{+}$por $U(\sigma, t) x=$ $u(\sigma, x, t)$. Um processo em $X$ é uma aplicação $u: \mathbb{R} \times X \times \mathbb{R}^{+} \rightarrow X$ satisfazendo as seguintes propriedades:

(i) u é contínua;

(ii) $U(\sigma, 0)=I$, a identidade;

(iii) $U(\sigma+s, t)=U(\sigma, s+t)$.

Um processo será dito autônomo, ou semigrupo, se $U(\sigma, t)$ independer de $\sigma$, ou seja, temos $T(t)=U(0, t)$. Note que o fluxo, definido no Capítulo 1 é um semigrupo. Considerando a equação (4.7) com condição inicial $y_{t}=\phi$ e supondo que esta admita uma única solução contínua $x(\sigma, \phi)(t)$, podemos definir um processo da seguinte maneira: $u(\sigma, \phi, \tau)=x_{\sigma+\tau}(\sigma, \phi)$.

Vamos, agora, definir o conceito de atração e atrator global para um processo. Esta é a Definição 4.6 .1 de [17].

Definição 4.12. Dados um processo u em $X$ e $\sigma \in \mathbb{R}$, diremos que um conjunto $M \subset$ $\mathbb{R} \times X$ atrai um conjunto $H \subset X$ em $\sigma$ se, para todo $\varepsilon>0$, existir um $t_{0}=t_{0}(\varepsilon, H, \sigma)$ tal que $(\sigma+t, U(\sigma, t) H) \subset B(M, \varepsilon)$ para $t \geq t_{0}(\varepsilon, H, \sigma)$, onde $B(M, \varepsilon)$ denota a $\varepsilon$ vizinhança do conjunto $M$. Diremos que $M$ atrai conjuntos limitados, se $M$ atrair cada conjunto limitado de $X$. Por fim, diremos que $M$ é um atrator global, se $M$ for invariante pelo processo e atrair conjuntos limitados de $X$.

Teorema 4.13. Considere a equação diferencial funcional em medida (4.7). Seja $U:\left[t_{0}-r,+\infty\right) \times \mathbb{R}^{n} \rightarrow \mathbb{R}$ uma função contínua à esquerda em $\left(t_{0}-r,+\infty\right)$. Suponha 
que os limites

$$
U(t-, y(t-))=\lim _{s \rightarrow t^{-}} U(s, y(s)), \quad t \in\left(t_{0}-r,+\infty\right)
$$

$e$

$$
U(t+, y(t+))=\lim _{s \rightarrow t^{+}} U(s, y(s)), \quad t \in\left[t_{0}-r,+\infty\right),
$$

existam, com $U(t-, y(t-))=U(t, y(t))$, para $y \in G^{-}\left(\left[t_{0}-r,+\infty\right), \mathbb{R}^{n}\right)$. Suponha que as condições $(i)$ e $($ ii $)$ do Teorema 4.10 estejam satisfeitas e que exista uma função $d: \mathbb{R}_{+} \rightarrow \mathbb{R}_{+}$de classe Hahn tal que para toda solução y de (4.7), tenhamos

$$
\sup _{\theta \in[-r, 0]} U(s+\theta, y(s+\theta)) \leq d(|y(s)|)
$$

onde $s \geq t_{0}$, com $d(\bar{t}) \geq b(\bar{t})$, para todo $\bar{t} \geq 0$. Suponha, ainda, que exista uma função $\Lambda: \mathbb{R}_{+} \rightarrow \mathbb{R}_{+}$que satisfaça $\Lambda(0)=0$ e $\Lambda(x)>0$, se $x \neq 0$, e que exista uma função contínua e não decrescente $p(s)>s$ para $s>0$ tal que

$$
D^{+} U(t, \psi(0)) \leq-\Lambda(|\psi(0)|) \quad \text { se } U(t+\theta, \psi(\theta))<p(U(t, \psi(0))
$$

para $\theta \in[-r, 0], t \in\left[t_{0},+\infty\right)$ e $\psi \in G^{-}\left([-r, 0], \mathbb{R}^{n}\right)$. Então a solução trivial $y \equiv 0$ de (4.7) será uniformemente assintoticamente estável. Se, além disso, a função b do teorema anterior satisfizer $b(s) \rightarrow \infty$ quando $s \rightarrow \infty$, então a solução $x=0$ será um atrator global para a equação diferencial funcional em medida (4.7).

A demonstração deste teorema é análoga àquela feita em [4], Teorema 4.9. Esta demonstração segue algumas ideias da demonstração feita em [17], Teorema 5.4.2. Nesta demonstração podemos observar que o fato de $b(s) \rightarrow \infty$ quando $s \rightarrow \infty$ implica que podemos fixar um $\delta$ arbitrário e determinar um $H$ tal que $d(\delta)=b(H)$ e, como demonstrado no Teorema 5.4.2 de [17], este fato e a estabilidade assintótica uniforme da solução nula, implicam que $x=0$ é um atrator global.

\subsection{Outros resultados de estabilidade}

Nesta seção, apresentaremos mais alguns resultados de estabilidade para EDFs em medida. Tais resultados são análogos aos resultados de [4], porém aqui tais resultados valem para o caso de EDFs em medida e em [4] valem apenas para EDFRs. 
Consideramos que as hipóteses sobre a equação (4.1) são as mesmas que foram descritas no início deste capítulo e que as hipóteses sobre a equação (4.7) são as mesmas da Seção 2 deste capítulo.

Apresentaremos primeiramente os resultados de estabilidade para EDOGs e, utilizando o Teorema 3.11, obteremos os resultados de estabilidade para equações em medida.

Todas as definições que serão utilizadas nesta seção, tais como estabilidade regular, atratividade regular, estabilidade assintótica regular e funcionais de Lyapunov, foram apresentadas anteriormente no Capítulo 3 e nas Seções 1 e 2 do Capítulo 4.

Antes do primeiro lema desta seção, precisamos de dois resultados auxiliares que serão enunciados aqui. O primeiro deles é o conhecido Lema de Saks-Henstock. Uma demonstração dele pode ser encontrada em [25], Lema 1.13, para $X=\mathbb{R}^{n}$. A demonstração para $X$ sendo um espaço de Banach arbitrário é análoga.

Lema 4.14. Seja $U:[a, b] \times[a, b] \rightarrow X$ Kurzweil integrável sobre $[a, b]$. Dado $\varepsilon>0$, seja $\delta$ um calibre em $[a, b]$ tal que

$$
\left\|\sum_{j=1}^{k}\left[U\left(\tau_{j}, s_{j}\right)-U\left(\tau_{j}, s_{j-1}\right)\right]-\int_{a}^{b} D U(\tau, t)\right\|<\varepsilon
$$

para toda divisão marcada $\delta$-fina $d=\left\{\left(\tau_{j},\left[s_{j-1}, s_{j}\right]\right), j=1,2, \ldots, k\right\}$ de $[a, b]$. Se

$$
a \leq \beta_{1} \leq \xi_{1} \leq \gamma_{1} \leq \beta_{2} \leq \xi_{2} \leq \gamma_{2} \leq \ldots \leq \beta_{m} \leq \xi_{m} \leq \gamma_{m} \leq b
$$

representar uma divisão parcial marcada $\delta$-fina $\left\{\left(\xi_{j},\left[\beta_{j}, \gamma_{j}\right]\right), j=1,2, \ldots, m\right\}$ de $[a, b]$, isto é,

$$
\xi_{j} \in\left[\beta_{j}, \gamma_{j}\right] \subset\left(\xi_{j}-\delta\left(\xi_{j}\right), \xi_{j}+\delta\left(\xi_{j}\right)\right), \quad j=1,2, \ldots, m
$$

então teremos

$$
\left\|\sum_{j=1}^{m}\left[U\left(\xi_{j}, \beta_{j}\right)-U\left(\xi_{j}, \gamma_{j}\right)-\int_{\beta_{j}}^{\gamma_{j}} D U(\tau, t)\right]\right\|<\varepsilon .
$$

Como consequência do Lema de Saks-Henstock, temos o seguinte resultado.

Corolário 4.15. Seja $U:[a, b] \times[a, b] \rightarrow X$ Kurzweil integrável sobre $[a, b]$. Dado $\varepsilon>0$, seja $\delta$ um calibre em $[a, b]$. Seja $[\gamma, v]$ um subintervalo fechado de $[a, b]$. Então, 
(i) $(v-\gamma)<\delta(\gamma)$ implica

$$
\left\|U(\gamma, v)-U(\gamma, \gamma)-\int_{\gamma}^{v} D U(\tau, t)\right\|<\varepsilon
$$

(ii) $(v-\gamma)<\delta(v)$ implica

$$
\left\|U(v, v)-U(v, \gamma)-\int_{\gamma}^{v} D U(\tau, t)\right\|<\varepsilon
$$

Agora somos capazes de demonstrar nossos resultados. O lema a seguir é uma generalização do Lema 10.12 de [25].

Lema 4.16. Seja $G \in \mathcal{F}(\Omega, h)$. Suponha que $V:\left[t_{0},+\infty\right) \times X \rightarrow \mathbb{R}$ seja tal que $V(\cdot, x):\left[t_{0},+\infty\right) \rightarrow \mathbb{R}$ é contínua à esquerda em $\left(t_{0},+\infty\right)$ para $x \in X$ e satisfaça

$$
|V(t, z)-V(t, y)| \leq K\|z-y\|, \quad z, y \in X, t \in\left[t_{0},+\infty\right)
$$

onde $K$ é uma constante positiva. Além disso, suponha que exista uma função $\Phi$ : $X \rightarrow \mathbb{R}$ tal que para toda solução $x:[a, b] \rightarrow X,[a, b] \subset\left[t_{0},+\infty\right)$, de (4.1), tenhamos

$$
\dot{V}(t, x(t))=\limsup _{\eta \rightarrow 0+} \frac{V(t+\eta, x(t+\eta))-V(t, x(t))}{\eta} \leq \Phi(x(t)), \quad t \in[a, b] .
$$

Se $\bar{x}:[\gamma, v] \rightarrow X, t_{0} \leq \gamma<v<+\infty$, for contínua à esquerda em $(\gamma, v]$ e de variação limitada em $[\gamma, v]$, então

$$
V(v, \bar{x}(v))-V(\gamma, \bar{x}(\gamma)) \leq K \sup _{s \in[\gamma, v]}\left(\left\|\bar{x}(s)-\bar{x}(\gamma)-\int_{\gamma}^{s} D G(\bar{x}(\tau), t)\right\| \|\right)+M(v-\gamma)
$$

onde $M=\sup _{t \in[\gamma, v]} \Phi(\bar{x}(t))$.

Demonstração: Seja $\bar{x}:[\gamma, v] \rightarrow X$ uma função contínua à esquerda em $(\gamma, v]$ e regrada em $[\gamma, v] \subset\left[t_{0},+\infty\right)$. Pela Proposição 1.5, a integral $\int_{\gamma}^{v} D G(\bar{x}(\tau), t)$ existe.

Tomemos $\sigma \in[\gamma, v]$. Pelo Teorema 1.6, a equação (4.1) admite uma solução local, digamos, $x:\left[\sigma, \sigma+\eta_{1}(\sigma)\right] \rightarrow X$ em $\left[\sigma, \sigma+\eta_{1}(\sigma)\right]$, satisfazendo a condição inicial $x(\sigma)=\bar{x}(\sigma)$. É claro que a integral $\int_{\sigma}^{\sigma+\eta_{1}(\sigma)} D G(x(\tau), t)$ existe.

Seja $\eta_{2}>0$ suficientemente pequeno tal que $\eta_{2} \leq \eta_{1}(\sigma)$ e $\sigma+\eta_{2} \leq v$. Então a integral $\int_{\sigma}^{\sigma+\eta_{2}} D G(x(\tau), t)$ existe e a integral $\int_{\sigma}^{\sigma+\eta_{2}} D[G(\bar{x}(\tau), t)-G(x(\tau), t)]$ também existe pela propriedade da integral de Kurzweil de integrabilidade em subintervalos. 
Portanto, dado $\varepsilon>0$, existe um calibre $\delta$ de $\left[\sigma, \sigma+\eta_{2}\right]$ correspondente a $\varepsilon$ na definição da última integral. Podemos supor, sem perda de generalidade, que $\eta_{2}<\delta(\sigma)$. Por (4.27), podemos tomar $0<\eta \leq \eta_{2}$ de forma que a desigualdade

$$
V(\sigma+\eta, x(\sigma+\eta))-V(\sigma, x(\sigma)) \leq \eta \Phi(x(\sigma))
$$

seja válida, e podemos supor, pelo Corolário 4.15(i), que

$$
\left\|G(\bar{x}(\sigma), \sigma+\eta)-G(\bar{x}(\sigma), \sigma)-\int_{\sigma}^{\sigma+\eta} D G(\bar{x}(\tau), t)\right\|<\frac{\eta \epsilon}{2 K}
$$

e

$$
\left\|G(x(\sigma), \sigma+\eta)-G(x(\sigma), \sigma)-\int_{\sigma}^{\sigma+\eta} D G(x(\tau), t)\right\|<\frac{\eta \epsilon}{2 K} .
$$

Note que

$$
\begin{gathered}
\left\|\int_{\sigma}^{\sigma+\eta} D[G(\bar{x}(\tau), t)-G(x(\tau), t)]\right\| \\
-\|G(\bar{x}(\sigma), \sigma+\eta)-G(\bar{x}(\sigma), \sigma)-G(x(\sigma), \sigma+\eta)+G(x(\sigma), \sigma)\| \\
\leq \| \int_{\sigma}^{\sigma+\eta} D[G(\bar{x}(\tau), t)-G(x(\tau), t)] \\
-(G(\bar{x}(\sigma), \sigma+\eta)-G(\bar{x}(\sigma), \sigma)-G(x(\sigma), \sigma+\eta)+G(x(\sigma), \sigma)) \| \\
\leq\left\|G(\bar{x}(\sigma), \sigma+\eta)-G(\bar{x}(\sigma), \sigma)-\int_{\sigma}^{\sigma+\eta} D G(\bar{x}(\tau), t)\right\| \\
+\left\|G(x(\sigma), \sigma+\eta)-G(x(\sigma), \sigma)-\int_{\sigma}^{\sigma+\eta} D G(x(\tau), t)\right\| .
\end{gathered}
$$

Como

$$
\begin{gathered}
\|G(\bar{x}(\sigma), \sigma+\eta)-G(\bar{x}(\sigma), \sigma)-G(x(\sigma), \sigma+\eta)+G(x(\sigma), \sigma)\| \\
\leq\|\bar{x}(\sigma)-x(\sigma)\||h(\sigma+\eta)-h(\sigma)|=0,
\end{gathered}
$$

uma vez que $\bar{x}(\sigma)=x(\sigma)$ e vale (1.4), segue por (4.30) e (4.31) que

$$
\left\|\int_{\sigma}^{\sigma+\eta} D[G(\bar{x}(\tau), t)-G(x(\tau), t)]\right\| \leq \frac{\eta \epsilon}{K} .
$$

Além disso, (4.26) implica

$$
V(\sigma+\eta, \bar{x}(\sigma+\eta))-V(\sigma+\eta, x(\sigma+\eta)) \leq
$$




$$
\begin{gathered}
\leq K\|\bar{x}(\sigma+\eta)-x(\sigma+\eta)\|=K\|\bar{x}(\sigma+\eta)-\bar{x}(\sigma)+x(\sigma)-x(\sigma+\eta)\| \\
=K\left\|\bar{x}(\sigma+\eta)-\bar{x}(\sigma)-\int_{\sigma}^{\sigma+\eta} D G(x(\tau), t)\right\| .
\end{gathered}
$$

Então (4.33), (4.29) e (4.32) implicam

$$
\begin{gathered}
V(\sigma+\eta, \bar{x}(\sigma+\eta))-V(\sigma, \bar{x}(\sigma)) \\
=V(\sigma+\eta, \bar{x}(\sigma+\eta))-V(\sigma+\eta, x(\sigma+\eta))+V(\sigma+\eta, x(\sigma+\eta))-V(\sigma, x(\sigma)) \\
\leq K\left\|\bar{x}(\sigma+\eta)-\bar{x}(\sigma)-\int_{\sigma}^{\sigma+\eta} D G(x(\tau), t)\right\|+\eta \Phi(x(\sigma)) \\
\leq K\left\|\bar{x}(\sigma+\eta)-\bar{x}(\sigma)-\int_{\sigma}^{\sigma+\eta} D G(x(\tau), t)\right\|+\eta M \\
\leq K\left\|\bar{x}(\sigma+\eta)-\bar{x}(\sigma)-\int_{\sigma}^{\sigma+\eta} D G(\bar{x}(\tau), t)\right\| \\
+K\left\|\int_{\sigma}^{\sigma+\eta} D[G(\bar{x}(\tau), t)-G(x(\tau), t)]\right\|+\eta M \\
\leq K\left\|\bar{x}(\sigma+\eta)-\bar{x}(\sigma)-\int_{\sigma}^{\sigma+\eta} D G(\bar{x}(\tau), t)\right\|+\eta \varepsilon+\eta M
\end{gathered}
$$

Dado $s \in[\gamma, v]$, definimos

$$
P(s)=\bar{x}(s)-\int_{\gamma}^{s} D G(\bar{x}(\tau), t) .
$$

Como $\bar{x}$ é regrada em $[\gamma, v]$, segue da Proposição 1.5 que $P$ também é regrada em $[\gamma, v]$. Então, temos

$$
\begin{aligned}
P(\sigma+\eta)-P(\sigma) & =\bar{x}(\sigma+\eta)-\bar{x}(\sigma)-\int_{\gamma}^{\sigma+\eta} D G(\bar{x}(\tau), t)+\int_{\gamma}^{\sigma} D G(\bar{x}(\tau), t) \\
& =\bar{x}(\sigma+\eta)-\bar{x}(\sigma)-\int_{\sigma}^{\sigma+\eta} D G(\bar{x}(\tau), t) .
\end{aligned}
$$

Agora, definimos

$$
f(t)=K\|P(t)-P(\sigma)\|+\varepsilon t+M t
$$


Note que

$$
\begin{aligned}
V(\sigma+\eta, \bar{x}(\sigma+\eta))-V(\sigma, \bar{x}(\sigma)) & \leq K\|P(\sigma+\eta)-P(\sigma)\|+\eta \varepsilon+\eta M \\
& =K(\|P(\sigma+\eta)-P(\sigma)\|-\|P(\sigma)-P(\sigma)\|) \\
& +\eta \varepsilon+\eta M=f(\sigma+\eta)-f(\sigma) .
\end{aligned}
$$

Por (4.35) e pela Proposição 4.2, obtemos

$$
\begin{aligned}
V(v, \bar{x}(v))-V(\gamma, \bar{x}(\gamma)) & \leq f(v)-f(\gamma) \\
& =K(\|P(v)-P(\sigma)\|-\|P(\gamma)-P(\sigma)\|)+\varepsilon(v-\gamma) \\
& +M(v-\gamma) \leq K\|P(v)-P(\sigma)-[P(\gamma)-P(\sigma)]\| \\
& +\varepsilon(v-\gamma)+M(v-\gamma)=K\|P(v)-P(\gamma)\|+\varepsilon(v-\gamma) \\
& +M(v-\gamma) \leq K\left(\left\|\bar{x}(v)-\int_{\gamma}^{v} D G(\bar{x}(\tau), t)-\bar{x}(\gamma)\right\|\right) \\
& +\varepsilon(v-\gamma)+M(v-\gamma) \\
& \leq K\left\|\bar{x}(v)-\bar{x}(\gamma)-\int_{\gamma}^{v} D G(\bar{x}(\tau), t)\right\| \\
& +\varepsilon(v-\gamma)+M(v-\gamma) .
\end{aligned}
$$

Como $\varepsilon>0$ é arbitrário, segue o resultado.

O próximo resultado nos dá condições sob as quais a solução nula de (4.1) é regularmente estável.

Teorema 4.17. Seja $V:\left[t_{0},+\infty\right) \times \overline{B_{\rho}} \rightarrow \mathbb{R}$ um funcional de Lyapunov, onde $\overline{B_{\rho}}=$ $\{y \in X:\|y\| \leq \rho\}, 0<\rho<c$. Suponha que $V$ satisfaça as seguintes condições adicionais:

(i) $V(t, 0)=0, t \in\left[t_{0},+\infty\right)$;

(ii) Existe uma constante $K>0$ tal que

$$
|V(t, z)-V(t, y)| \leq K\|z-y\|, \quad t \in\left[t_{0},+\infty\right), z, y \in \overline{B_{\rho}} .
$$

Então a solução trivial $x \equiv 0$ de (4.1) será regularmente estável. 
Demonstração: Seja $\bar{x}:[\gamma, v] \rightarrow X$ uma função regrada em $[\gamma, v] \subset\left[t_{0},+\infty\right)$. Pela condição (iii) da Definição 4.4 e pelo Lema 4.16, temos

$$
V(t, \bar{x}(t)) \leq V(\gamma, \bar{x}(\gamma))+K \sup _{s \in[\gamma, t]}\left\|\bar{x}(s)-\bar{x}(\gamma)-\int_{\gamma}^{s} D G(\bar{x}(\tau), t)\right\|,
$$

para todo $t \in[\gamma, v]$.

Como $V$ é um funcional de Lyapunov, existe uma função $b: \mathbb{R}^{+} \rightarrow \mathbb{R}^{+}$de classe Hahn tal que

$$
V(t, x) \geq b(\|x\|), \quad(t, x) \in[\gamma, v] \times \bar{B}_{\rho} .
$$

Sejam $\varepsilon>0$ e $b(\varepsilon)>0$. Seja $\delta(\varepsilon)>0$ tal que $2 K \delta(\varepsilon)<b(\varepsilon)$. Se

$$
\sup _{s \in[\gamma, t]}\left\|\bar{x}(s)-\bar{x}(\gamma)-\int_{\gamma}^{s} D G(\bar{x}(\tau), t)\right\|<\delta(\varepsilon),
$$

então

$$
\begin{aligned}
V(t, \bar{x}(t)) & \leq V(\gamma, \bar{x}(\gamma))++K \sup _{s \in[\gamma, t]}\left\|\bar{x}(s)-\bar{x}(\gamma)-\int_{\gamma}^{s} D G(\bar{x}(\tau), t)\right\| \\
& \leq|V(\gamma, \bar{x}(\gamma))|+K \frac{\delta(\varepsilon)}{2}+K \frac{\delta(\varepsilon)}{2} \\
& \leq K\|\bar{x}(\gamma)\|+K \delta(\varepsilon) \leq 2 K \delta(\varepsilon)<b(\varepsilon), t \in[\gamma, v] .
\end{aligned}
$$

Por outro lado, suponha que exista $u \in[\gamma, v]$ tal que $\|\bar{x}(u)\| \geq \varepsilon$. Então, por (4.37), temos

$$
V(u, \bar{x}(u)) \geq b(\|\bar{x}(u)\|) \geq b(\varepsilon),
$$

o que contradiz (4.38). Logo $\|\bar{x}(t)\|<\varepsilon$ para $t \in[\gamma, v]$ e o resultado segue.

O próximo teorema mostra que, com uma condição a mais sob o funcional de Lyapunov, a solução nula da EDOG (4.1) é regularmente assintoticamente estável.

Teorema 4.18. Seja $V:\left[t_{0},+\infty\right) \times \overline{B_{\rho}} \rightarrow \mathbb{R}$ um funcional de Lyapunov, onde $\overline{B_{\rho}}=$ $\{y \in X:\|y\| \leq \rho\}, 0<\rho<c$. Suponha que $V$ satisfaça a condições $(i) e(i i) d o$ Teorema 4.1\%. Além disso, suponha que exista uma função contínua $\Phi: X \rightarrow \mathbb{R}$, satisfazendo $\Phi(0)=0$ e $\Phi(x)>0$ para $x \neq 0$, tal que para toda solução $x:[\gamma, v] \rightarrow B_{\rho}$ de (4.1), com $[\gamma, v] \subset\left[t_{0},+\infty\right)$, tenhamos

$$
\dot{V}(t, x(t)) \leq-\Phi(x(t)), \quad t \in[\gamma, v]
$$

Então a solução trivial $x \equiv 0$ de (4.1) será regularmente assintoticamente estável. 
Demonstração: Como todas as hipóteses do Teorema 4.17 estão satisfeitas, a solução trivial $x \equiv 0$ de (4.1) é regularmente estável. Resta mostrarmos que a solução $x \equiv 0$ de (4.1) é regularmente atratora.

Como a solução $x \equiv 0$ de (4.1) é regularmente estável, temos

(I) Existe $\widetilde{\delta} \in(0, \rho)$ tal que se $\bar{x}:[\gamma, v] \rightarrow B_{c},[\gamma, v] \subset\left[t_{0},+\infty\right)$, é uma função regrada em $[\gamma, v]$, tal que

$$
\|\bar{x}(\gamma)\|<\frac{\widetilde{\delta}}{2}
$$

e

$$
\sup _{s \in[\gamma, v]}\left\|\bar{x}(s)-\bar{x}(\gamma)-\int_{\gamma}^{s} D G(\bar{x}(\tau), t)\right\|<\widetilde{\delta},
$$

então

$$
\|\bar{x}(t)\|<\rho, \quad t \in[\gamma, v]
$$

(II) Para todo $\varepsilon>0$, existe $\delta=\delta(\varepsilon)>0, \delta<\varepsilon$ tal que, se $\bar{x}:[\bar{\gamma}, \bar{v}] \rightarrow B_{c}$, $[\bar{\gamma}, \bar{v}] \subset\left[t_{0},+\infty\right)$, é uma função regrada em $[\bar{\gamma}, \bar{v}]$, tal que

$$
\|\bar{x}(\bar{\gamma})\|<\delta
$$

$\mathrm{e}$

$$
\sup _{s \in[\bar{\gamma}, \bar{v}]}\left\|\bar{x}(s)-\bar{x}(\gamma)-\int_{\bar{\gamma}}^{s} D G(\bar{x}(\tau), t)\right\|<\delta,
$$

então

$$
\|\bar{x}(t)\|<\varepsilon, \quad t \in[\bar{\gamma}, \bar{v}] .
$$

Seja

$$
N=\sup \{-\Phi(y): \delta(\varepsilon) \leq\|y\| \leq \varepsilon\}=-\inf \{\Phi(y): \delta(\varepsilon) \leq\|y\| \leq \varepsilon\}<0
$$

Defina

$$
T(\varepsilon):=\min \left\{v-\gamma,-K \frac{\delta_{0}+\delta(\varepsilon)}{N}\right\}>0 .
$$

Suponha que $\bar{x}:[\gamma, v] \rightarrow B_{c},[\gamma, v] \subset\left[t_{0},+\infty\right)$, seja uma função regrada em $[\gamma, v]$, tal que

$$
\|\bar{x}(\gamma)\|<\frac{\widetilde{\delta}}{2}
$$

e

$$
\sup _{s \in[\gamma, v]}\left\|\bar{x}(s)-\bar{x}(\gamma)-\int_{\gamma}^{s} D G(\bar{x}(\tau), t)\right\|<\delta(\varepsilon) .
$$


Queremos mostrar que

$$
\|\bar{x}(t)\|<\varepsilon, \quad t \in[\gamma, v] \cap[\gamma+T(\varepsilon),+\infty), \gamma \geq t_{0}
$$

Afirmamos que existe $t^{*} \in[\gamma, \gamma+T(\varepsilon)]$ tal que $\left\|\bar{x}\left(t^{*}\right)\right\|<\delta(\varepsilon)$. Suponha o contrário, ou seja, $\|\bar{x}(s)\| \geq \lambda(\varepsilon)$, para qualquer $s \in[\gamma, \gamma+T(\varepsilon)]$. Pelo Lema 4.16, pelas condições (i) e (ii) do Teorema 4.17, (4.42), (4.40) e (4.41), obtemos

$$
\begin{aligned}
V(\gamma+T(\varepsilon), \bar{x}(\gamma+T(\varepsilon))) & \leq V(\gamma, \bar{x}(\gamma))+K \sup _{s \in[\gamma, \gamma+T(\varepsilon)]}\left\|\bar{x}(s)-\bar{x}(\gamma)-\int_{\gamma}^{s} D G(\bar{x}(\tau), t)\right\| \\
& +N T(\varepsilon)<K \delta(\varepsilon)+K \frac{\widetilde{\delta}}{2}+N\left(-K \frac{\widetilde{\delta}+\delta(\varepsilon)}{N}\right) \\
& \leq K\|\bar{x}(\gamma)\|+K \delta(\varepsilon)+K \frac{\widetilde{\delta}}{2}+N\left(-K_{2} \frac{\widetilde{\delta}+\delta(\varepsilon)}{N}\right) \\
& =K \frac{\widetilde{\delta}}{2}-K \widetilde{\delta}<K \widetilde{\delta}-K \widetilde{\delta}=0 .
\end{aligned}
$$

Por outro lado, como $V$ é um funcional de Lyapunov, existe uma função $b: \mathbb{R}_{+} \rightarrow$ $\mathbb{R}_{+}$de classe Hahn tal que

$$
V(t, x) \geq b(\|x\|), \quad \text { para }(t, x) \in\left[t_{0},+\infty\right) \times \overline{B_{\rho}} .
$$

Assim,

$$
V(\gamma+T(\varepsilon), \bar{x}(\gamma+T(\varepsilon))) \geq b(\|\bar{x}(\gamma+T(\varepsilon))\|) \geq b(\lambda(\varepsilon))>0
$$

o que contradiz (4.3). Então segue nossa afirmação. Portanto, $\|\bar{x}(t)\|<\varepsilon$ para $t \in$ $\left[t^{*}, v\right]$, já que (II) vale para $\bar{\gamma}=t^{*}$ e $\bar{v}=v$. Também, $\|\bar{x}(t)\|<\varepsilon$ para $t>\gamma+T(\varepsilon)$, já que $t^{*} \in[\gamma, \gamma+T(\varepsilon)]$ e, portanto, a solução $x \equiv 0$ da equação (4.1) é regularmente atratora e o resultado segue.

Os teoremas que virão a seguir são similares aos Teoremas 4.8 e 4.9. Porém, note que aqui precisamos de mais hipóteses, especificamente, a hipótese de que o funcional de Lyapunov satisfaça uma condição do tipo lipschitz na segunda variável. Faremos as demonstrações destes teoremas já que eles são generalizações para o caso de uma EDF em medida dos Teoremas 4.6 e 4.7 de [4]. Antes de demonstrar tais teoremas precisamos de um lema.

Lema 4.19. Considere a equação diferencial funcional em medida (4.7). Suponha que a função $U:\left[t_{0},+\infty\right) \times \bar{E}_{\rho} \rightarrow \mathbb{R}$ satisfaça as seguintes condições: 
(i) $U(t, 0)=0, t \in\left[t_{0},+\infty\right)$;

(ii) Existe uma constante $K>0$ tal que

$$
|U(t, \psi)-U(t, \bar{\psi})| \leq K\|\psi-\bar{\psi}\|, \quad t \in\left[t_{0},+\infty\right), \quad \psi, \bar{\psi} \in \bar{E}_{\rho}
$$

Então a função $V:\left[t_{0},+\infty\right) \times \bar{B}_{\rho} \rightarrow \mathbb{R}$ definida por (4.8) satisfaz $V(t, 0)=0$ para todo $t \in\left[t_{0},+\infty\right)$, e

$$
|V(t, z)-V(t, \bar{z})| \leq K\|z-\bar{z}\|
$$

para $t \geq t_{0} e z, \bar{z} \in \bar{B}_{\rho}$.

Uma demonstração deste lema para o caso de uma EDFR pode ser encontrado em [4], Lema 4.4. A demonstração para o caso de uma EDF em medida é análoga.

Teorema 4.20. Considere a equação diferencial funcional em medida (4.7). Suponha que $U:\left[t_{0},+\infty\right) \times \bar{E}_{\rho} \rightarrow \mathbb{R}$ seja um funcional de Lyapunov e que as seguintes condições sejam válidas:

(i) $U(t, 0)=0, t \in\left[t_{0},+\infty\right)$;

(ii) Existe uma constante $K>0$ tal que

$$
|U(t, \psi)-U(t, \bar{\psi})| \leq K\|\psi-\bar{\psi}\|, \quad t \in\left[t_{0},+\infty\right), \quad \psi, \bar{\psi} \in \bar{E}_{\rho} .
$$

Então a solução trivial $y \equiv 0$ de (4.7) será uniformemente estável.

Demonstração: Esta demonstração segue as ideias da demonstração do Teorema 4.6 de [4].

Como $f$ satisfaz as condições $\left(H_{1}\right)$ até $\left(H_{3}\right)$ do Capítulo 3 , a função $F$ na equação (4.1) pertence à classe $\mathcal{F}(\Omega, h)$, com $\Omega=O \times\left[t_{0},+\infty\right) \operatorname{com} h:\left[t_{0},+\infty\right) \rightarrow \mathbb{R}$ dada por

$$
h(t)=\int_{t_{0}}^{t}[M(s)+L(s)] d g(s), \quad t \in\left[t_{0},+\infty\right) .
$$

Seja $V:\left[t_{0},+\infty\right) \times \bar{B}_{\rho} \rightarrow \mathbb{R}$ dada por (4.8). Pelo Lema 4.19,

$$
V(t, 0)=0, \quad t \in\left[t_{0},+\infty\right)
$$

e

$$
\mid V(t, z)-V(t, \bar{z}) \leq K\|z-\bar{z}\|, \quad t \in\left[t_{0},+\infty\right), z, \bar{z} \in \bar{B}_{\rho} .
$$


Como visto anteriormente, $V$ é um funcional de Lyapunov e as hipóteses do Teorema 4.17 estão satisfeitas. Então a solução $x \equiv 0$ de (4.1) é regularmente estável.

Logo para todo $\varepsilon>0$, existe $\delta=\delta(\varepsilon)>0$ tal que se $\bar{x}:[\gamma, v] \rightarrow B_{\rho}, t_{0} \leq \gamma<v<$ $+\infty$ for uma função regrada que satisfaz

$$
\|\bar{x}(\gamma)\|<\delta \quad e \quad \sup _{s \in[\gamma, v]}\left\|\bar{x}(s)-\bar{x}(\gamma)-\int_{\gamma}^{s} D G(\bar{x}(\tau), t)\right\|<\delta,
$$

então

$$
\|\bar{x}(t)\|<\varepsilon, \quad t \in[\gamma, v]
$$

Sejam $\phi \in \bar{E}_{\rho}$ e $\bar{y}:\left[t_{0}-r,+\infty\right) \rightarrow \mathbb{R}^{n}$ uma solução de (4.7) tal que $\bar{y}_{t_{0}}=\phi$ e suponhamos que

$$
\|\phi\|<\delta
$$

Queremos mostrar que

$$
\left\|\bar{y}_{t}\left(t_{0}, \phi\right)\right\|<\varepsilon, \quad t \in\left[t_{0},+\infty\right)
$$

Denotamos $\bar{y}_{t}=\bar{y}_{t}\left(t_{0}, \phi\right)$ e definimos

$$
\bar{x}(t)(\tau)=\left\{\begin{array}{l}
\bar{y}(\tau), t_{0}-r \leq \tau \leq t \\
\bar{y}(t), \tau \geq t
\end{array}\right.
$$

Pelo Teorema 3.11, $\bar{x}$ é uma solução em $\left[t_{0},+\infty\right)$ da EDOG (4.1) satisfazendo a condição inicial $\bar{x}\left(t_{0}\right)=\widetilde{x}, \operatorname{com}$

$$
\widetilde{x}(\tau)= \begin{cases}\phi\left(\tau-t_{0}\right), & t_{0}-r \leq \tau \leq t_{0} \\ \phi(0), & \tau \geq t_{0}\end{cases}
$$

Além disso, $\bar{x}$ é regrada. Por (4.44) e (4.47),

$$
\left\|\bar{x}\left(t_{0}\right)\right\|=\sup _{t_{0}-r \leq \tau<+\infty}|\widetilde{x}(\tau)|=\|\phi\|<\delta .
$$

Também, para todo $v \in\left(t_{0},+\infty\right)$,

$$
\sup _{s \in\left[t_{0}, v\right]}\left\|\bar{x}(s)-\bar{x}(\gamma)-\int_{t_{0}}^{s} D G(\bar{x}(\tau), t)\right\|=\sup _{s \in\left[t_{0}, v\right]}\left\|\bar{x}\left(t_{0}\right)\right\|<\delta .
$$


Portanto, (4.43) vale, ou seja, $\|\bar{x}(t)\|<\varepsilon$ para todo $t \in\left[t_{0}, v\right]$. Logo (4.46) implica que, para qualquer $t \in\left[t_{0}, v\right]$,

$$
\begin{aligned}
\left\|\bar{y}_{t}\left(t_{0}, \phi\right)\right\| & =\left\|\bar{y}_{t}\right\|=\sup _{-r \leq \theta \leq 0}|\bar{y}(t+\theta)| \leq \sup _{t_{0}-r \leq \tau \leq v}|\bar{y}(\tau)| \\
& =\sup _{t_{0}-r \leq \tau \leq v}|\bar{x}(v)(\tau)|=\sup _{t_{0}-r \leq \tau<+\infty}|\bar{x}(v)(\tau)| \\
& =\|\bar{x}(v)\|<\varepsilon .
\end{aligned}
$$

Como $v$ é arbitrário, (4.45) segue, o que completa a demonstração.

Teorema 4.21. Considere a equação diferencial funcional em medida (4.7). Suponha que $U:\left[t_{0},+\infty\right) \times \overline{E_{\rho}} \rightarrow \mathbb{R}$ seja um funcional de Lyapunov e que satisfaça as condições (i) e (ii) do Teorema 4.20. Além disso, suponha que exista uma função contínua $\Lambda: \mathbb{R}_{+} \rightarrow \mathbb{R}_{+}$tal que $\Lambda(0)=0$ e $\Lambda(x)>0$ se $x \neq 0$, de forma que, para qualquer $\psi \in \bar{E}_{\rho}$, tenhamos

$$
D^{+} U(t, \psi) \leq-\Lambda(\|\psi\|), \quad t \geq t_{0}
$$

Então a solução trivial y $\equiv 0$ de (4.7) será uniformemente assintoticamente estável.

Demonstração: Esta demonstração segue as ideias da demonstração do Teorema 4.7 de [4]. Seja $V:\left[t_{0},+\infty\right) \times \bar{B}_{\rho} \rightarrow \mathbb{R}$ dado por (4.8). Então as hipóteses do Teorema 4.17 estão satisfeitas.

Definimos $\Phi: \bar{B}_{\rho} \rightarrow \mathbb{R}$ por $\Phi(z)=\Lambda(\|z\|)$. Então $\Phi$ é contínua, $\Phi(0)=0$ e $\Phi(z)>0, z \neq 0$.

Suponhamos que $x:\left[t_{0},+\infty\right) \rightarrow \bar{B}_{\rho}$ seja uma solução de (4.1) tal que $(x(t))_{t}=\psi$, $t \in\left[t_{0},+\infty\right)$ e $\psi \in \bar{E}_{\rho}$ e consideramos $y:\left[t_{0}-r,+\infty\right) \rightarrow \mathbb{R}^{n}$ a solução de (4.7), dada pelo Teorema 3.11, que satisfaz $y_{t}=\psi$. Por (4.51),

$$
\begin{gathered}
\limsup _{\eta \rightarrow 0+} \frac{V\left(t+\eta, x_{\psi}(t+\eta)\right)-V\left(t, x_{\psi}(t)\right)}{\eta}=D^{+} U\left(t, y_{t}(t, \psi)\right)= \\
=D^{+} U(t, \psi) \leq-\Lambda(\|\psi\|)=-\Lambda\left(\left\|y_{t}\right\|\right) .
\end{gathered}
$$

Também temos

$$
\left\|y_{t}\right\|=\left\|x_{\psi}(t)\right\|
$$

Portanto,

$$
\limsup _{\eta \rightarrow 0+} \frac{V\left(t+\eta, x_{\psi}(t+\eta)\right)-V\left(t, x_{\psi}(t)\right)}{\eta} \leq-\Lambda\left(\left\|y_{t}\right\|\right)=-\Lambda\left(\left\|x_{\psi}(t)\right\|\right)=-\Phi\left(x_{\psi}(t)\right)
$$


e as hipóteses do Teorema 4.18 estão satisfeitas. Então a solução $x \equiv 0$ de (4.1) é regularmente assintoticamente estável, isto é, existe $\delta_{0}>0$ e para todo $\varepsilon>0$, existem $T=T(\varepsilon) \geq 0$ e $\rho=\rho(\varepsilon)>0$ tal que se $\bar{x}:[\gamma, v] \rightarrow B_{c}, t_{0} \leq \gamma<v<+\infty$ for uma função regrada tal que

$$
\|\bar{x}(\gamma)\|<\delta_{0}
$$

$\mathrm{e}$

$$
\sup _{s \in[\gamma, v]}\left\|\bar{x}(s)-\bar{x}(\gamma)-\int_{\gamma}^{s} D G(\bar{x}(\tau), t)\right\|<\rho,
$$

então

$$
\|\bar{x}(t)\|<\varepsilon, \quad t \in[\gamma, v] \cap[\gamma+T,+\infty), \gamma \geq t_{0}
$$

Dado $\varepsilon>0$, sejam $\delta_{0}, \rho>0$ e $T=T(\varepsilon)$ como acima. Seja $\phi \in E_{c}$ e consideramos $\bar{y}:\left[t_{0}-r,+\infty\right) \rightarrow \mathbb{R}^{n}$ solução de $(4.7)$ tal que $\bar{y}_{t_{0}}=\phi \mathrm{e}$

$$
\|\phi\|<\delta_{0}
$$

Queremos mostrar que

$$
\left\|\bar{y}_{t}\left(t_{0}, \phi\right)\right\|<\varepsilon, \quad t \in\left[t_{0}+T,+\infty\right)
$$

Mas isto segue como na demonstração do Teorema 4.20. Por (4.55), obtemos (4.52) como em (4.48). Também, como no Teorema 4.20, temos (4.49) e, portanto, segue (4.53). Finalmente segue (4.56), já que temos (4.50), como no Teorema 4.20 e (4.54). 
atratividade com respeito a perturbações, 48

atratividade integral, 34

atratividade regular, 47

Bacia de atração EDOGs, 14

Bacia de atração EDOs, 25

calibre, 2

Classe $\mathcal{F}(\Omega, h), 3$

Classe $\widetilde{\mathcal{F}}(\Omega, h), 5$

Dependência contínua de soluções EDOG, 12

Dependência contínua EDOs, 24

divisão marcada, 2

divisão marcada $\delta$-fina, 2

EDF em medida perturbada, 33

EDOG autônoma, 8

equação diferencial funcional em medida, 29

equação diferencial ordinária generalizada

(EDOG), 2

estabilidade assintótica com respeito a perturbações, 48

estabilidade assintótica uniforme, 59 estabilidade com respeito a perturbações, 48

estabilidade de Lyapunov, 33, 58

estabilidade integral, 34

estabilidade integral assintótica, 35

estabilidade regular, 47

estabilidade regular assintótica, 47

estabilidade uniforme, 33, 58

estabilidade uniforme assintótica, 33

Existência-unicidade EDFs em medida, 45

Existência-unicidade soluções EDOGs, 7 Existência-unicidade soluções EDOs, 22

fluxo, 13

fluxo no tempo, 13

funcional de Lyapunov EDOGs autônomas, 16

funcional de Lyapunov EDOs autônomas, 26

integral de Kurzweil, 2

Integral de Kurzweil-Henstock-Stieltjes, 30

integral de Kurzweil-Henstock-Stieltjes estendida, 31

ponto de equilíbrio, 13 
ponto de equilíbrio assintoticamente es-

tável, 14

ponto de equilíbrio estável, 14

ponto de equilíbrio instável, 14

propriedade do prolongamento, 32

solução EDF em medida, 34

solução EDO autônoma, 21 



\section{Referências Bibliográficas}

[1] S. M. Afonso, Equações diferenciais funcionais com retardamento e impulsos em tempo variável via equações diferenciais ordinárias generalizadas, Tese de doutorado, ICMC-USP, 2011. (Citado na página xiii.)

[2] S. M. Afonso. E. M. Bonotto, M. Federson, On exponential stability of functional differential equations with variable impulsive perturbations, submetido para publicação. (Citado nas páginas xiii e 2.)

[3] S. M. Afonso, E. M. Bonotto, M. Federson, L. P. Gimenes, Boundedness of solutions for functional differential equations with variable impulses via generalized ordinary differential equations, Math Nach., 285(5-6) (2012), 545-561. (Citado nas páginas 2 e 59.)

[4] S. M. Afonso; E. Bonotto; M. Federson; L. P. Gimenes, Stability of functional differential equations with variable impulsive perturbations via generalized ordinary differential equations, submetido para publicação. (Citado nas páginas 57, 60, 69, $70,71,79,80$ e 82.$)$

[5] S. M. Afonso, E. M. Bonotto, M. Federson, Š. Schwabik, Discontinuous local semiflows for Kurzweil equations leading to LaSalles invariance principle for differential systems with impulses at variable times, J. Differential Equations 250(7) (2011), 2969-3001 (Citado nas páginas xii, 9, 10, 11 e 12.)

[6] J. M. Arrieta, A. N. Carvalho, Abstract Parabolic Problems With Critical Nonlinearities And Applications To Navier-Stokes And Heat Equations, Transactions of the American Mathematical Society, v. 352, n. 01 (2000), p. 285-310. (Citado na página xiv.) 
[7] Z. Artstein, Topological dynamics of an ordinary differential equation and Kurzweil equations, J. Differential Equations, 23 (1977), 224-243. (Citado nas páginas xi e xii.)

[8] P. C. Das, R. R. Sharma, Existence and stability of measure differential equations, Czech. Math. Journal 22(97) (1972), 145-158 (Citado na página 29.)

[9] M. Federson, J.G. Mesquita, A. Slavik, Basic results for functional differential and dynamic equations involving impulses, submetido para publicação. (Citado na página xiv.)

[10] M. Federson, J. G. Mesquisa, A. Slavík, Measure functional differential equations and functional dynamic equations on time scales, J. Differential Equations, v. 252 (2012), p. 3816-3847. (Citado nas páginas xiv, 2, 29, 33, 37 e 45.)

[11] M. Federson, J. G. Mesquita, E. Toon, Stability results for measure functional differential equations via Kurzweil-equations, submetido para publicação. (Citado nas páginas 35,36 e 37.)

[12] M. Federson, Š. Schwabik, Generalized ODEs approach to impulsive retarded functional differential equations, Differential and Integral Equations., 19(11), (2006), 1201-1234. (Citado nas páginas xii, 2, 5, 7 e 24.)

[13] M. Federson, Š Schwabik, Stability for retarded functional differential equations, Ukranian Math. J. 60(1) (2008), 121-140. (Citado nas páginas xiii e 2.)

[14] M. Federson, P. Z. Táboas, Topological dynamics of retarded functional differential equations, J. Differential Equations, 195(2), (2003), 313-331. (Citado nas páginas xii, xiii e 2.)

[15] M. Federson, E. Toon, A new approach to autonomous retarded functional differential equations with impulses, pre-print. (Citado na página 23.)

[16] A. Halanay, Differential Equations: Stability, Oscillations, Time Lags. Academic Press, New York, 1966. (Citado na página 34.)

[17] J. K. Hale, S. M. V. Lunel, Introduction to Functional Differential Equations. Applied Mathematical Sciences, Springer-Verlag (1991). (Citado nas páginas 33, 69, 70 e 71.$)$

[18] R. Henstock, Lectures on the Theory of Integration, World Scientific, Singapore, 1988. (Não citado.) 
[19] C. S. Hönig, Volterra Stieltjes Integral Equations, North Holland Publ. Comp., Amsterdam, 1975. (Citado nas páginas 4, 10 e 11.)

[20] C. S. Hönig, As integrais de gauge (de Kurzweil, de Henstock e de McShane), Minicurso do 37 Seminário Brasileiro de Análise (1993). (Citado na página 31.)

[21] C. Imaz and Z. Vorel, Generalized ordinary differential equations in Banach spaces and applications to functional equations, Bol. Soc. Mat. Mexicana, 11 (1966), 47-59. (Citado na página xii.)

[22] J. Kurzweil, Generalized ordinary differential equations and continuous dependence on a parameter, Czechoslovak Math. J. 7(82) (1957), 418-448. (Citado nas páginas xi e 2.)

[23] J. Kurzweil, Generalized ordinary differential equations, Czechoslovak Math. J. 8(83) (1958), 360-388. (Citado na página 2.)

[24] F. Oliva and Z. Vorel, Functional equations and generalized ordinary differential equations, Bol. Soc. Mat. Mexicana, 11 (1966), 40-46. (Citado na página xii.)

[25] Š. Schwabik, Generalized Ordinary Differential Equations, World Scientific, Series in Real Anal., vol. 5, 1992. (Citado nas páginas xi, xii, 2, 3, 4, 16, 30, 31, 59, 60, 72 e 73.)

[26] Š. Schwabik, Variational stability for generalized ordinary differential equations, Časopis Pěst. Mat. 109(4) (1984), 389-420. (Não citado.)

[27] Š. Schwabik, Abstract Perron-Stieltjes integral, Math. Bohem., 121 (1996), 425447. (Citado na página 2.) 